\title{
Smoking cessation among patients hospitalized with cardiac disease: evaluation of a minimal-contact intervention
}

Citation for published version (APA):

Bolman, C. A. W. (2001). Smoking cessation among patients hospitalized with cardiac disease: evaluation of a minimal-contact intervention. [Doctoral Thesis, Maastricht University]. Datawyse / Universitaire Pers Maastricht. https://doi.org/10.26481/dis.20011109cb

Document status and date:

Published: 01/01/2001

DOI:

10.26481/dis.20011109cb

Document Version:

Publisher's PDF, also known as Version of record

Please check the document version of this publication:

- A submitted manuscript is the version of the article upon submission and before peer-review. There can be important differences between the submitted version and the official published version of record.

People interested in the research are advised to contact the author for the final version of the publication, or visit the DOI to the publisher's website.

- The final author version and the galley proof are versions of the publication after peer review.

- The final published version features the final layout of the paper including the volume, issue and page numbers.

Link to publication

\footnotetext{
General rights rights.

- You may freely distribute the URL identifying the publication in the public portal. please follow below link for the End User Agreement:

www.umlib.nl/taverne-license

Take down policy

If you believe that this document breaches copyright please contact us at:

repository@maastrichtuniversity.nl

providing details and we will investigate your claim.
}

Copyright and moral rights for the publications made accessible in the public portal are retained by the authors and/or other copyright owners and it is a condition of accessing publications that users recognise and abide by the legal requirements associated with these

- Users may download and print one copy of any publication from the public portal for the purpose of private study or research.

- You may not further distribute the material or use it for any profit-making activity or commercial gain

If the publication is distributed under the terms of Article 25fa of the Dutch Copyright Act, indicated by the "Taverne" license above, 
SMOKING CESSATION AMONG PATIENTS

HOSPITALIZED WITH CARDIAC DISEASE:

EVALUATION OF A MINIMAL-CONTACT INTERVENTION 



\section{SMOKING CESSATION AMONG PATIENTS HOSPITALIZED WITH CARDIAC DISEASE:}

\section{EVALUATION OF A MINIMAL-CONTACT INTERVENTION}

\section{PROEFSCHRIFT}

ter verkrijging van de graad wan doctor aan de Universiteit Maastricht, op gezag van de Rector Magnificus, Prof. dr. A.C. Nieuwenhuijzen Kruseman volgens het besluit van het College van Decanen, in het openbaar te verdedigen op vrijdag 9 november 2001 om 16.00 uur

door Catherine Adriana Wilhelmina Bolman

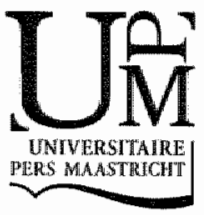




\section{Promotor:}

Prof. dr. H. de Vries

Beoordelingscommissie:

Prof. dr. N.K. de Vries (voonitter)

Prof, dr. H.W van den Borne

Prof. dr. GiJ. Kok

Dr. A.N. Mudde

Dr. M. Willemsen (Stichting Volksgezondheid en Roken, Den Haag) 
Het onderzoek dat aan dit proefschrift ten grondslag ligt, is mogelik gemaakt door een gezamenlijke subsidie wan de Nederlandse Hartstichting

(NHS-subsidienummer p45001) en de Stichting Volksgezondheid en Roken.

Ontwerp \& lay-out: Z\&D, Niek Daniëls, Bladel

ISBN: $90-5278-3209$

Universitaire pers Maastricht 


\section{CONTENTS}

Chapter 1 General introduction 9

Introduction and outline of the thesis 9

Outline of the $A B C$ planning model 11

The ABC planning madel applied to cardiac inpatients 12

Chapter 2 Psycho-social determinants and motivational phases

in smoking behavior of cardiac inpatients 35

Abstract $\quad 35$

Introduction $\quad 36$

Methods 39

Results $\quad 41$

Discussion $\quad 45$

Conclusions 48

Chapter 3 Evaluation of a nurse-managed minimal-contact smoking cessation intervention for cardiac impatients 51

Abstract 51

Introduction $\quad 52$

Methods 53

Results 60

Discussion 67

Chapter 4 A minimal-contact intervention for cardiac inpatients: long-term effects on smoking cessation 71

Abstract 71

Intraduction $\quad 72$

Methods 73

Results $\quad 78$

Discussion $\quad 85$

Chapter 5 Factors determining cardiac nurses' intentions

to continue using a smoking cessation protocol 91

Abstract 91

Introduction $\quad 92$

Methods 94

Results 99

Discussion $\quad 102$ 
Chapter 6 Main conclusions and discussion 105 Abstract 105

Summany and discussion of major findings $\quad 106$

Methodological issues $\quad 114$

Recommendations for further research $\quad 117$

Recommendations for improvement of the C-MIS 121

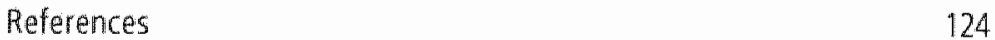

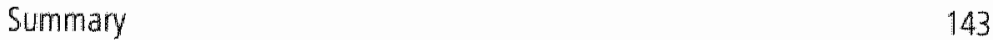

Samenvatting $\quad 147$

Notes 151

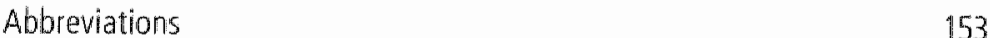

Curriculum Vitae 154

Acknowledgments 155 


\section{Chapter one}

\section{GENERAL INTRODUCTION}

\section{Introduction and Outline of the Thesis}

In industrialized countries, cardiovascular disease (CVD), which involves diseases of the heart and blood vessels, is the main cause of montality Wonld Health Organization, 1999). Coronay heart disease (CHD), which concerns diseases of the heart's blood circulation system (e.g., myocardial infarction), is mainly responsible for the high CWD mortality and is the leading single cause of death Murray \& Lopez, 1996; World Health Organization, 1999).

Decades of researchi on the epidemiology of CVO and CHD have resulted in a body of evidence that lifestyle factors such as smoking, eating too much saturated fat and being physically inactive play an important role in the development, progression and curation of these types of diseases (Jansen, 1994; McGinnis \& Foege, 1993; Sparrow \& Dawber, 1978; U.S. Department of Health \& Human Services [U.5. DHHS], 1990; World Health Organization, 1994).

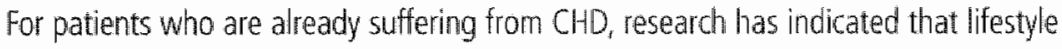
changes such as smoking cessation can effectively extend their overall surviwal rate, improve their quality of life, decrease their need for expensive interventional revascularization procedures', and can prevent further deterioration of their cardiac condition (see for example Pearson et al., 1994; Smith et al., 1995). It has furthermore been claimed that smoking cessation improves a patient's prognosis more than any other treatment (Deckers et al., 1994; Mehta \& Eagle, 1998; Simith, 1997; U.S. DHHS, 1990). Management of $\mathrm{CHD}$, however, is still primary directed toward medical treatment. During the past two decades, for example, new technologies such as coroniary angioplasty (PTCA)', coronary artery bypass surgery $(\mathrm{CABG})^{3}$ and medication therapies have become accepted treatment standards, whereas lifestyle modification has received far less attention.

The present thesis describes the development and evaluation of a smoking cessation intervention for Dutch patients hospitalized with CHD. The importance of a cessation program for these patients can be derived from the following arguments: (1) It has frequently been reported that most patients persist in or resume smoking after a coronary event ${ }^{4}$ (see for example Deckers et al. 1994; EUROASPIRE Study Group, 1997). In this respect, a task force of the European society of Cardiology has feported that the success rate of smoking cessation among CHD patients is far from satisfactory and calls for structural efforts by health care providers (EUROASPIRE Study Group, 1997). (2) Dutch research and the experience of a working group of the Netherlands Society for Cardiovascular Nursing indicate that the smoking behavior of patients 
hospitalized at cardiology wards has so far received minimal attention (NIPO, 1995; Wiebing \& Baan, 1997). (3) Although the international literature reports on several smoking cessation interventions amed at hospitalized CHD patients (Condon, 1997; Johnson, Budz, Mackay, \& Miller, 1999; Ockene, Kristeller, Goldberg, Ockene et al., 1992; Orleans, Rotberg, Quade, \& Lees, 1990; Rigotti, Mckool, \& Shiffman, 1994; Rosal et al., 1998; Taylor, Houston Miller, Killen, \& DeBusk, 1990), no such study had been carried out in the Netherlands.

Because the effectiveness of such a health promotion intervention is influenced by the quality of its planning (Green \& Kreuter, 1999; Kok, Van den Borne, \& Mullen, 1997; Tones \& Tiltord, 1994, the project described in the present thesis was developed according to principles of health promotion planning models. More specifically, the $\mathrm{ABC}$ planning model served as the framework (De Vries, 1998; De Vries \& Kok, 1996). The next section of this chapter includes a brief explanation of this model, while the final section describes how the $A B C$ planning model was applied to the problem of smoking among hospitalized CHD patients. In addition, this final section is meant to provide an overview of the existing literature on the subject.

Chapter two describes a study of the stages of change in smoking cessation and the underlying psycho-social determinants among hospitalized cardiac patients. The short and long term behavioral effects of the intervention are described in the chapters three and four. Chapter five reports on a study of nurses" intentions and underlying motives to continue the use of the smoking cessation protocol developed in the present research project. In chapter six, the main findings, conclusions and implications of the studies described in this thesis are discussed.

Because most chapters were written to be published as research papers, they are self-explanatory outside the context of this thesis. Due to this structure, some information will be provided more than once. Table 1.1 provides an overview of the studies that were conducted in context of the present thesis.

\section{Table 1.1 Overview of the studies discussed in this thesis}

\begin{tabular}{|c|c|c|c|c|}
\hline Chapter & Design & $\begin{array}{l}\text { Method of } \\
\text { data collection }\end{array}$ & Sample & Study objectiwe \\
\hline wo & cross-sectional & questionnaire & 532 & $\begin{array}{l}\text { stages (phases) of change and psycho- } \\
\text { social feterminants of smokng cessation }\end{array}$ \\
\hline threet & longifutina & questionnaire & 789 & $\begin{array}{l}\text { effects of intervention on smoking } \\
\text { cessation after thiree months alnd process } \\
\text { evaluation }\end{array}$ \\
\hline foul & lorgitudinal & questionmaire & 789 & $\begin{array}{l}\text { effects of intervention on smoking cessation } \\
\text { after twelve months }\end{array}$ \\
\hline five & cross-sectional & questionnaire & 85 & $\begin{array}{l}\text { psycho-social determinanis of continued } \\
\text { use of the protocol by nurses }\end{array}$ \\
\hline
\end{tabular}




\section{Outline of the ABC Planning Model}

The $A B C$ planning model can be wiewed as a comprehensive model for systematically planning health education and promotion activities (De Vries, 1998; De Vries \& Kok, 1996). It is an integration of several planning models that have been used in the fieid of health education and promotion. It is based on the Precede-Proceed framework (Green \& Kreuter, 1999), its simplified version by Kok (Damoiseaux \& Kok, 1993), the Community Change Model by Bracht (1999) and Roger's theory on the diffusion of innovations (Rogers, 1995). It distinguishes three phases (see Figure 1.1): (1) Analysis of the problem, (2) Behavioral change and, (3) Continuation of behavioral change. The figure shows that the planning process has been conceptualized as a circular process. Depending on the situation, a program developer has to decide where to begin, and it may sometimes be necessary to employ activities in various phases simultaneously.

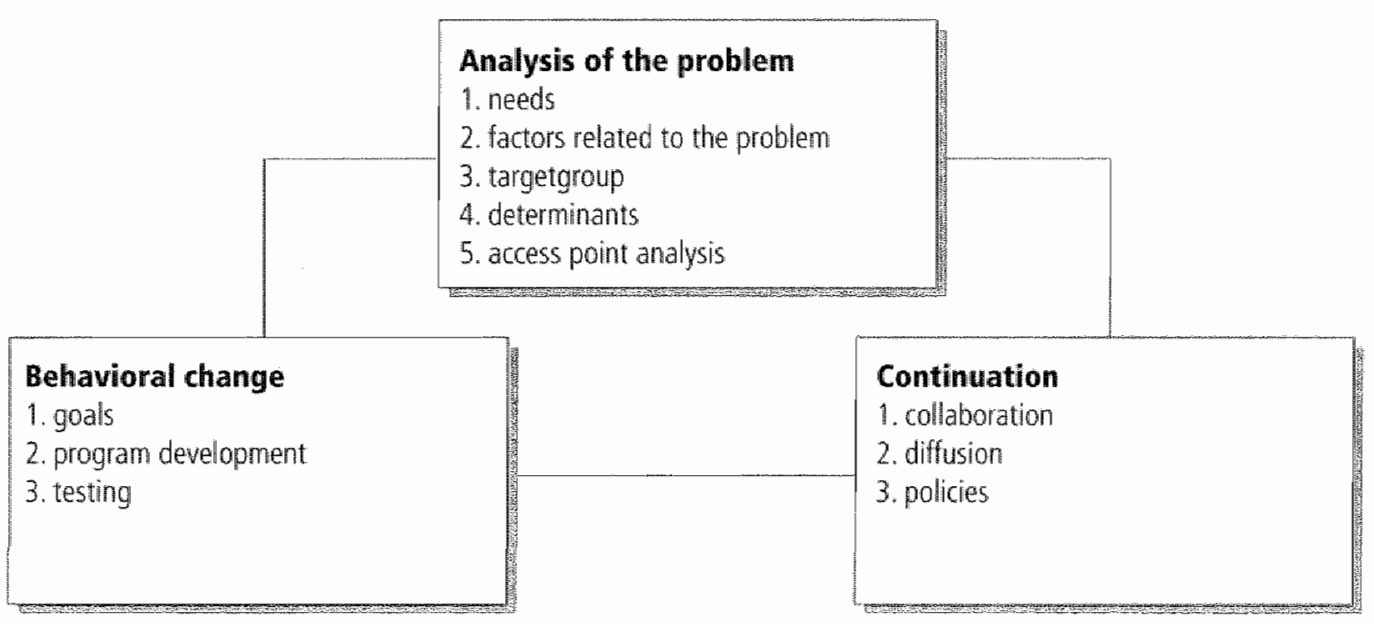

Figure 1.1. The ABC planning model (De Vries \& Kok, 1996).

The first phase is intended to analyze a (health) problem thorouginly by means of five steps. The first step concerns an assessment of the needs related to the reduction of social or health problems or both. As suggested by Green and Kreuter (Green \& Kreuter, 1999), two scenarios are possible. In an ideal situation, the starting point is a consideration of the quality of life as perceived by a population and the identification of problems that threaten this quality of life. More often, however, a health problem has alleady been selected and information on its extent, seriousness and threat has to be obtained. The second step involves an analysis of behavioral and environmental factors that are related to the problem(s) defined in the previous step. Subsequently, decisions have to be made about the factors that are to be addressed in an intervention. Then, a 
target group has to be defined (step 3). This should be the group of people in whom the risk behavior is most prevalent and is not yet being targeted. The fourth step is to identify the intentions and motives of the target group to engage in a desired behavior and to understand the reasons for persisting in an unhealthy behavior. This step is necessary to find out how the target group can be motivated to change the risk behavior. The final step is to analyze how the target group can best be reached.

The second phase involves the formulation of goals, the development of a program and the testing of its efficacy or effectiveness. Goals need to be formulated at several levels. General program goals state who is expected to achieve how much improvement or change of what health condition by when (health gain). Behavioral change objectives specify who is expected to perform how much of what behavior by when. Motivational and mastery objectives state what the target group will have to learn in order to achieve the behavioral change. Motivational objectives refer to cognitive changes that have to occur, while mastery objectives aim at skills acquisition. The second step involves the actual development of a program which should be based on theoretical insights and prior research. As the most immediate objectives, the motivational and mastery objectives provide the starting point for the intervention, leading to the accomplishment of the behavioral change objectives, and eventually the achievement of general program goals (Bartholomew, Parcel, \& Kok, 1998). Step three concerns the evaluation of the program.

The third phase is needed to facilitate dissemination of an intervention that has proved to be effective. It consists of three steps, which do not necessary have to be consecutive. One step concerns the development of inter-sectoral collaboration and support, which is necessary to achieve commitment by the stakeholders involved (Bracht, 1999; De Vries, 1998). Stakeholders need to be identified and invited to represent their interest group in a collaboration group, which is also referred to as a "linkage approach" (Orlandi, Landers, Weston, \& Haley, 1990). It is advocated to form such a group at a very early stage of the project. Second, diffusion involves an assessment of stimulating and hindering factors perceived by program users regarding the adoption. implementation and continuation of the program. This allows one to develop strategies. that facilitate the diffusion of the program. Third, it is often necessary to create supportive policies that increase the chances of successful program diffusion. Hospitals should for example, provide clinicians with adequate resources to enable them to dellver a smoking cessation program.

\section{The ABC Planning Model Applied to Cardiac Inpatients}

This section illustrates how the $A B C$ planning model was applied to the problem of smoking among cardiac inpatients. For this purpose, an overview of the analysis of the health problem, the strategies for behavioral change, and the strategies for continuation of the intervention is provided. 


\section{Analysis of the Health Problem}

\section{Needs Assessment.}

In this subsection, the problem of smoking among CHD patients is reviewed in terms of its extent, severity and related risk factors. This first requires an explanation of the difference between CVD and CHD. CVD refers to all diseases of the human blood circulation system such as high blood pressure, rheumatic heart disease, peripheral vascular disease, coronary heart disease and cerebrovascular diseases (World Health Organization, 1992). By contrast, CHD refers specifically to diseases of the heart's blood circulation system characterized by stenosis of the coronary arteries., and includes: acute myocardial infarction, other "sub)acute forms of ischemic heart disease, old myocardial infarction, angina pectoris, and other forms of chronic ischemic heart diseases (World Health Organization, 1992).

In the Netherlands, CVD is the leading cause of death, with almost $40 \%$ of overall mortality (Konings-Dalstra \& Reitsma, 1999). Thirty-eight percent of CVD mortality is caused by CHD. Other heart diseases (e.g., decompensatio cordis) and cerebrovascular diseases also have an important part in CVD mortality, both with 24\% (Konings-Dalstra \& Reitsma, 1999). Europe in general and the United States show comparable figures (Eurostat, 1997; Murray \& Lopez, 1996).

Of all hospital admissions in the Netherlands in 1993, almost one-fifth were CVD. related. CHD was responsible for one-third of the total number of hospital admissions for CVD (Reitsma, 1995), a figure which did not change in 1999 (Konings-Dalstra \& Reitsma, 1999).

CVD and CHD are associated with enormous costs of care, loss of productivity, and disability. For example, in the Netherlands CVD is the second largest source of costs in health care (Ruwaard \& Kramers, 1997). Its costs have shown one of the greatest rise of all disease in recent years and are expected to rise with more than $40 \%$ over the next 15 years (Post \& 5tokx, 1997; Ruwaard \& Kramers, 1997). For CHD, comparable figures are reported (Ruwaard \& Kramers, 1997). The huge volume of CHD treatments may be illustrated by the fact that Dutch hospitals perform about 14,500 coronary bypass surgeries ${ }^{3}$ and 13,500 Precutane Transluminal Coronary Angioplasties" every year (Dalstra \& Reitsma, 1997). Diagnostic procedures and drug therapies also attribute to the enormous costs related to these diseases.

Besides the fact that CVD, and more specifically CHD has a negative impact on sociely as a whole, the quality of life of individuals is also adwersely affected for a period of time. An acute heart attack or a coromary bypass operation causes uncertainty and psychological distress, not only during hospitalization, but also after discharge. In this respect, feelings of distress, depression, anxiety, hastility, fear of recurrence or progression of the disease, pain and uncertainty about the future have been frequently reported in the literature (Crumlish, 1994; Doeffler, Pbert, \& DeCosimo, 1994; Falger, 
Sebregts, Leuteren, \& Bär, 2000; Jaarsma, Kastemans, Dassen, \& Philipsen, 1995; Moser 8 Dracup, 1995; Moser, Dracup, \& Marsden, 1993; Stein, Troudart, Hymowitz, Gotsman, \& Kaplan De-Nour, 1990; Theobald, 1997). Research has also shown that recowery, especially psycho-social recovery, is a lengthy process (Erdman, Duivenvoorden, Verhage, Kazemier, \&ugenholtz, 1984; Havik \& Maeland, 1990; Moser \& Dracup, 1995). In sum. CVD and CHD are still major health problems which adversely affect society as a whole as well as individuals.

\section{Problem factors.}

This subsection highlights the relationship between CHD and smoking. Other risk factors related to CHD will only be addressed briefly, because this thesis focuses on smoking. Risk factors for CVD are not described explicitly, because CVD is not the focus of this thesis and its risk factors are generally comparable to those for CHD.

With respect to the factors that contribute to the development and manifestation of CHD, there is a body of evidence that lifestyle behaviors play an important role, besides risk factors such as genetic background, diabetes mellitus, hypertension, elevated serum cholesterol levels and personality factors (e.g., type A behavior) (Erica Research Group, 1991; Ruwaard \& Kramers, 1997; Sandvik et al. 1993; Schneiderman \& Skyler, 1996; U.S. Department of Health \& Human Services [U.S. DHHSH, 1983; Vlietstra, Kronmal, Oberman, Frye, \& Killip, 1986). A meta-analysis of a large number of epjdemiological studies on CHD mortality revealed Population Attributive Risks (PARs) among the $20-59$ years age group of $40 \%, 23 \%$ and $11 \%$ for smoking, physically inactive behavior and eating to much saturated fat respectively. In the same order, PARs for persons aged 60 years or over are $30 \%, 36 \%$ and $9 \%$ (Ruwaard \& Kramers, 1997 ). The Dutch Foundation on smoking and Health (Stivoro) reported that $31 \%(n=6015)$ of all CHD-related deaths in 1998 could be attributed to smoking (Dutch Foundation on Smoking \& Health, 1999). With respect to the relationship between smoking and CHD, the 1983 report of the U.S. Surgeon General stated: "Cigarette smoking is a major cause of CHO in the United States for both men and women and should be considered as the most important of the known modifiable risk factors for CHD" (U.S. DHHS, 1983, p. 6). In the same report, it was concluded that smokers have a $70 \%$ excess death rate from $\mathrm{CHO}_{n}$ while heavier smoker's have an even greater excess risk. Longitudinal studies on the relationship between cigarette smoking and CHD have found a relative risk of 2.6 for smokers in comparison with non-smokers (Doll. Peto, Wheatley, Gray, \& Sutherland, 1994; Shaten, Kuller " \& Neaton, 1991).

Positive effects of quitting smoking before clinical manifestations of CHD occur have also been studied extensively. The increased risk of CHD occurrence and death caused by smoking is reduced by $50 \%$ after one year of smoking abstinence and then declines gradually (U.S. DHHS, 1990). After 15 years of abstinence, the risk of CHD is similar to that 
of a person who has never smoked (U.5. DHHS, 1990). Doll and colleagues (1994) confirmed these conclusions on the basis of a 40 -year study among British doctors.

Smoking cessation also has positive effects on the prognosis of patients with already established CHD. Research has revealed that it improves the prognosis of cardiac patients more than any other treatment (Deckers et al. 1994; Mehta \& Eagle, 1998; Smith, 1997; U.S. DHHS, 1990). A meta-analysis has shown that smoking cessation reduces the risk of mortality after one year by almost $50 \%$ in patients with newly established CHD (Deckers et al. 1994). The risk reduction was comparable in men and women and in younger as well as in elderly patients. (Deckers et al., 1994). Similar results were found in two studies with, respectively, five and eleven years of follow-up (Gupta, Gupta, Sharma, \& Gupta, 1993; Vlietstra et al., 1986). A review of the effects of smoking cessation after a first myocardial infarction (M) or angina pectoris (AP) on morbidity revealed that quitting smoking reduces the risk of recurrence by half and also reduces the risk of new non-fatal complications (Mulcahy, 1983). Continuing to smoke after an invasive treatment procedure like PTCA or CABG also affects recovery adversely. Research has revealed that smoking persistence is positively associated with recurrence of stenosis ${ }^{5}$ after PTCA (Block, 1990) and after CABG /Solymoss, Nadeau, Millette, \& Campeau, 1988). A recently conducted Dutch study confirmed that repeat PTCA and CABG are more prevalent among persistent smokers compared to patients who have quit smoking (Domburg et al., 2000). A 15-year follow-up study of the effects of smoking cessation on recovery from CABG showed a $40 \%$ lower mortallty likelihood and an almost $60 \%$ reduced risk of a new $M$ (Voors et al. 1996). Most of the results described here were recently confirmed in a review (Van Berkel, Boersma, Roos-Hesselink, Erdman, \& Simoons, 1999b). Finally, one study reported lower hospitalization rates among patients who stopped smoking after a coronary event (Vlietstra et al., 1986 ).

\section{Target group.}

The previous two subsections showed that $\mathrm{CHD}$ is an important health problem, whose development, progression, and treatment are greatly alfected by cigarette smoking. As our smoking cessation intervention aimed at hospitalized CHD patients, the extent to which these patients engaged in risky behavior needed to be examined. Furthermore, before developing an intervention, we had to check whether these patients were not already being sufficiently approached lor their smoking behavior. This subsection therefore provides an overview of the smoking behavior of cardiac inpatients (target group at the micro level) and the efforts of ward nurses and cardiologists (the target group at the meso level) to the smoking behavior of their patients. 


\section{Smoking behavior of cardiac inpatients}

The target group at the micro level consisted of patients who smoked and had been admitted at cardiology wards of Dutch hospitals. With regard to the smoking behavior of cardiac inpatients immediately before the disease manifested itself, a retrospective study carried out in nine European countries, including the Netherlands, found an averaged $34 \%$ of smokers (EUROASPIRE Study Group, 1997). This study reported $41 \%$ smokers among Dutch CHD patients, which is considerably higher than the smoking prevalence rate of $34 \%$ among a similar age group (50-65 years) in the general Dutch population (Dutch Foundation on Smoking \& Health, 1999\%. Among Swedish patients admitted with a MI, 40\% were smokers before the event (Carlsson, Lindberg. Westin, Israelsson, 1997). An American study among patients who became hospitalized with a first MI reported a much higher smoking prevalence: $60 \%$ (Havik \& Maeland, 1988). The difference could be due to the fact that the latter study only included patients admitted for a first MI, whereas the other studies referred to above also included patients with a longer history of $\mathrm{CHD}$ or with other $\mathrm{CHD}$ manifestations. Another explanation could be that the American study was conducted ten years ago, when the smoking prevalence was higher (Peto, Lopez, Borham, Thun, \& Heath, 1994).

With respect to the smoking habits after the manifestation of CHD, the EUROASPIRE study showed that the percentage of Dutch cardiac inpatients persisting in their smoking behavior after an event was larger than that among the other European populations. Dutch CHD patients showed a smoking prevalence rate of $32 \%$ six months after an event, compared to 12 to $25 \%$ in the other countries (ELROASPIRE Study Group, 1997). The reported rate for Dutch patients was comparable to that found in another study among Dutch citizens diagnosed with a heart disease at some moment in their lives (Statistics Netherlands, 1997). Quit rates of 60\%, measured three month after a first non-fatal MI, were reported in two American studies (Havik \& Maeland, 1988; Marshail, 1990). Another American study showed 32\% cessation after six months and $25 \%$ after twelwe months (Rigotti, Singer, Mulley, \& Thibault, 1991). A review of the cessation behavior of CHD patients, measured 1-6 years after a first non-fatal myocardial event, showed an average cessation rate of $45 \%$ (Deckers \& Agema, 1994). Although the reported quit rates relate to spontaneous quitting after an event, it should be noticed that these patients could have been advised to quit.

\section{Smoking cessation assistance by nurses and cardiologists}

At the begining of the project described in this thesis, a working group of the Netherlands Society for Cardiovascular Nursing expressed their concern about the at. tention usually given to the smoking behavior of cardiac inpatients. Although the im. portance of assisting $\mathrm{CHD}$ patients to quit smoking is often stressed by nurses and such assistance is regarded as a normal part of their work, they experienced a lack of systematic attention to patients' smoking behavior in daily practice (personal communication). Their experiences were in line with findings of Dutch research, which showed that only one-third of the cardiology ward nurses and two-thirds of the cardiologists 
informed patients about the dangers of smoking and advised them to quit (NPO, 1995; Wiebing \& Baan, 1997). Moreover, it was reported that only $10 \%$ of both actually gu: ded their patients an smoking cessation, as was also found in the U.S. CDuncan, Stein, \& Cummings, 1991; Goldstein, Hellier, Fitzgerald, Stegall, \& Fischer, 1987; Sanders, Stone, Fowler, \&arzillier, 1986). Among Australian nurses, 36\% stated that they asked the majority of the patients for their smoking status. Only $10 \%$ assessed their smoking history and intentions to quit or discussed strategies for quitting (Nagle Schofield, \& Redman, 1999).

According to a study among physicians at a Dutch University hospital, only $25 \%$ of the physicians had experience of working with smoking cessation programs (Waalkens, Cohen-Schotanus, Adriaanse, \& Knol, 1992). Interviews collected in the EUROASPIRE study among CHD patients who still were smoking $1-6$ years after an event showed that $92 \%$ of them had at some stage been advised to stop smoking, in which roughly two-thirds had been advised before the event, compared to one third during the hospital stay. Seventy percent of the patients had received the advice from a hospital physiclan, while $50 \%$ had received it from a general practitioner (Van Berkel, Boersma, De Baquer, Deckers, \& Wood, 1999a). Unfortunately, these figures were not categorized by country. The study also reported that cardiologists had referred patients to a specialized smoking cessation program in less than 10\% of the cases. Pre-event smoking habits had not been documented in medical records in $20 \%$ of the cases, while in $65 \%$ of the cases the post event smoking habits had not been documented (Van Berkel et all, 1999a).

In sum, it can be concluded that the attention intermediaries pay to the smoking behavior of their patients is capable of improvement.

\section{Analysis of determinants.}

If we are to influence the risk behavior successfully, it is necessary to obtain insight into the motives of the target group to engage of persist in a particular behavior (De Vries, 1998; Green \& Kreuter, 1999). Because this is true for changing the smoking behavior of patients as well as for changing the educational behavior of the intermediaries, it is important to have insight into the interitions and behavioral determinants of both groups. Both are therefore examined below.

Determinants of smoking cessation among cardiac inpatients

Insight into the determinants of smoking cessation among cardiac inpatients were obtained by combining general knowiedge deriwed from wo social psychological modells, namely the ASE model (De Vries, Dijkstla, \& Kuhlman, 1988; De Vries \& Mudde, 1998; Kok, De Vries, Mudde, \& Strecher, 1991) and the Transtheoretical model (Prochaska \& Diclemente, 1983; Prochaska, Diclemente, \&orcross, 1992), with the literature 
on cardiac patients intentions and motives to quit. Social psychalogical models formed the theoretical basis because they focus on highly proximal and immediate causes of behavior, which are in contrast to more distal causes of behavior (e.g., personality) more easily changed (Conner \& Norman, 1996; Flay \& Petraitis, 1994).

The ASE model and Transtheoretical model were chosen because they have proved their applicability to the process of smoking cessation in a body of empirical research among several target groups (e.g., pregnant women, general population, employees) (De Vries \& Backbite, 1994; De Vries \& Mudde, 1998; De Vries, Mudde, Dijkstra, \& Willemsen, 1998; Dijkstra, De Vries, \& Bakker, 1996; Prochaska \& DiClemente, 1992; Prochaska \& Velicer, 1997; Willemsen, De Vries, Van Breukelen, \& Oldenburg, 1996).

The ASE model (see Figure 1.2) originated in the Theory of Reasoned Action (Fishbein \& Ajzen, 1975), its successor the Theory of Planned Behavior (Aizen, 1991), and Bandura's Social Cognitive Theory (Bandura, 1986). The model postulates that behavior can be predicted by behavioral intention, which in tum is influenced by proximal determinants like attitudes, perceived social influences and self-efficacy expectations. The impact of the three proximal determinants is assumed to be moderated by distal or extemal factors such as psychological factors (e.g., anxiety, personality) and biological factors (e.g., age, gender) (De Vries \& Mudde, 1998). The ASE model assumes that changes in the psycho-social determinants lead to changes in behavior, which is in line with assumptions in comparable social psychological models in the domain of behavioral change (Eagly \& Chaiken, 1993; Norman, Conner, \& Bell, 1999). Attitude can be described as an expression of a person's positive and negative evaluations about performing a given behavior (Ajzen, 1991; De Vries, Barkbier, Kok, \& Dijkstra, 1995). Social influence refers to the influence a person perceives, concerning the behavior, from his or her direct environment. Operationalizations of this concept vary from social norms, perceived behavior in a person's direct environment (modeling) and perceived support or pressure (De Viries et al., 1995). Self-efficacy refers to a person's judgement of his or her capability to perform a certain behavior (Bandura, 1986).

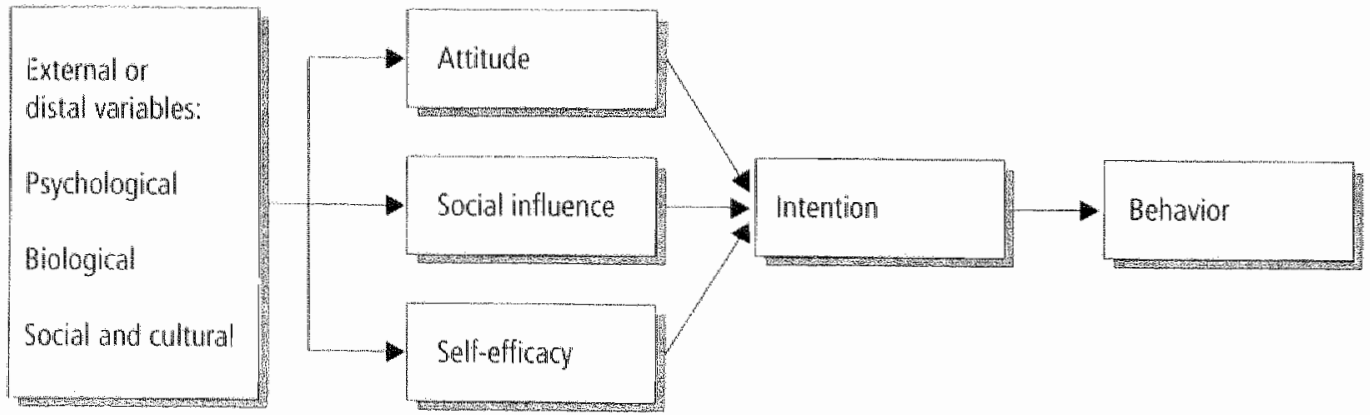

Eigure 1.2. ASE model (adapted from De Vries \& Mudde, 1998). 
The Transtheoretical Model has frequently and consistently showh hat smoking cessation can be regarded as a process in which people move from no motivation to quit to intemalization of the non-smoking behavior (Diclemente et al, 1991; Prochaska et al. 1992; Prochaska \&aldstein. 1991; Prochaska \& Velicer, 1997). This process proceeds along five stages of change $(\mathrm{SOC})$. In the first stage, precontemplation, people have no intentions to change their behawor. In contemplation, a person is considering behavioral change within six months. Preparators are actively planning to take action in the immediate future, usually within the next month. Subsequently, people can move into the action stage, in which they actually enact the new behavior. If people persist in the new behavior for over six monthis, they proceed to the maintenance stage (Prochaska et al, 1992). The model furthermore postulates that moving through the phases involves a cycling and recycling process in which people can relapse and revert to earlier stages or phases ${ }^{6}$ (Prochaska, 1991; Prochaska et at., 1992). In addition, research by Prochaska and colleagues (1994a; 1994b) showed that attitude and selfefficacy expectations with regard to the behavioral change differ for the various phases. They reported that precontemplators experienced more negative (cons) than positive (cons) comsequences of the behavior in question, whereas contemplators antcipated more pros of change. People in the action phase anticipated more pros and had higher self-efficacy expectations than people in the precontemplation phase. An American study confirmed this process of change for cardiac patients (Kristeller, Rossi, Ockene, Goldberg, \& et al., 1992)

Dutch studies integrating the SOC concept with the ASE model also showed that attitudes, social influences and selfefficacy were of different importance in the various phases (De Vries \& Backbier, 1994; De Vries et al. 1998; Dilkstra et al., 1996). The pattern found in these studies was labeled as the opattern (see Figure 1.3), and showed that smokers in the precontemplation phase can be distinguished from contemplators and actors by their negative attitude toward quitting. Actors showed higher levels of self-efficacy expectations than precontemplators and contemplators, whereas the perception of social influence was found to increase steadily from precontemplattion to action. A longitudinal study analyzing the factors that could predict phase tran" sition confirmed the 0 patterm and showed that positive attitudes were particularly more important in predicting the transition from precontemplation to contemplation. whereas self-efficacy was more predictive for the transition from contemplation to preparation and action (De Vries \& Mudde, 1998). The researchers who conducted these studies suggested that a stage-tailored information supply could help people to proceed through the change process. 


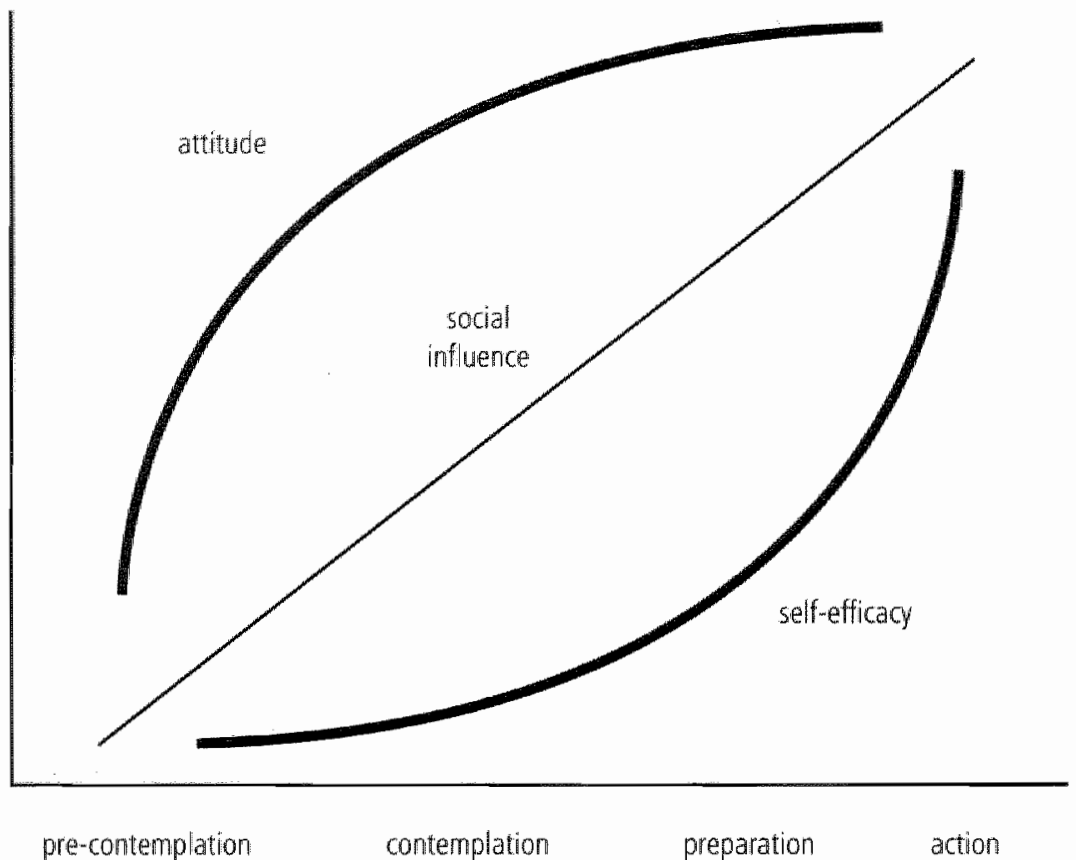

Fiqure 13. The Dattern

Two American studies have provided insight into cardiac patients' readiness to quit smoking. According to Ockene and colleagues (1992), 54\% of the hospitalized CHD patients were already in the action phase from the day of admission, while $31 \%$ were contemplators and 15\% precontemplators. A study by Kristeller and colleagues (1992) showed that hospitalized CHD patients were more often in the action phase than a non-medical population. Studies, reporting only on the quitting intentions showed that cardiac patients felt a great desire to quit (Emmons \& Goldstein, 1992; Orleans et al., 1990; Taylor et al., 1990). A Dutch study among patients visiting the cardiology outpatient clinic showed that $87 \%$ of the patients did not want to continue smoking as they had used to, although only a few wanted to quit within seven days (Breteler, Mertens, \& Rombouts, 1988). Although the literature shows ambivalent results with regard to the psycho-social predictors of quitting among this patient group, partly due to differences in methods and measurements, it has generally been found that patients who have strong intentions to quit and who have a positive attifude toward quitting, a supportive environment and strong self-efficacy expectations, are more likely to stop smoking (Ockene et al.. 1992; Orleans et al., 1990; Rice et al., 1996; Rigotti et al., 1994; Rosal et al., 1998; Taylor et al., 1990; Van Berkel, Vlugt, \& Boersma, 2000). Several studies also reported on other factors that were related to smoking cessation among $\mathrm{CHO}$ patients. In terms of the ASE model, such factors could be labeled as distal or external factors (see also figure 1.2). The disease itself has consistently been reported as the strongest 
predictor of quitting (Breteler et al., 1988; Glasgow, Stevens, Vogt, Mullooly, \& et al., 1991; Goodman. Nadkarni, \& Schorling, 1998; Marshall, 1990; McKenna \& Higgens, 1997; Ockene et al. 1992). Other predictors were: nore severe CHD (Ockene et al. 1992), first admission for CHD (Rigotti et al., 1991), recent hospitalization (Freund, D'Agostino, Belanger, Kannel, \& Stokes, 1992; Frid et al., 1991; Ockene et al., 1985). older age (Freund et al., 1992; Frid et al., 1991; Glasgow et al., 1991; Ockene et al. 1985; Taylor, Miller, Herman, Smith, \& et al., 1996), higher level of education (Freund et al., 1992; Frid et al., 1991; Ockene et al., 1985; Rosal et al., 1998), low state-anxiety levels and no depressive feelings. (Huijbrechts et a less nicotine dependence (Orleans et al., 1990; Rigotti et al., 1997; Rigotti et al., 1991). Most of these factors were also reported in a review paper on the predictors of smoking cessation among CHD patients (McKenna \& Higgens, 1997). Contrary findings were reported on the influence of previous quit attempts (Breteler et al., 1988; Greenwood, Muir, Packham, \& Madeley, 1995; Rigotti et al., 1994).

As regards the development of a smoking cessation intervention for cardiac inpatients, the following conclusions can be drawn from the general behavioral explanation models presented.

(1) People are more likely to quit smoking successfully if they have a positive attitude toward changing, if they perceive their direct environment as supportive (social influence) and if they perceive themselves as capable of changing and coping with highrisk situations (self-efficacy).

(2) The psycho-social determinants mentioned in the previous point differ between the phases of change in which a person is situated (e.g., people in the precontemplation phase need to get a positive attitude toward quitting, whereas contemplators need self-efficacy information to convert their plans into actions). As a consequence, the motivational and mastery objectives for achieving behavioral change in terms of smoking abstinence, differ for the various phases of change. This ineans, for example, that the main objective for precontemplators is to develop a positive attitude toward quitting, while for contemplators and actors, the objectives emphasize achieving higher self-efficacy expectations, removing barriers and the acquisition of skills.

The above overview of studies among CHD patients shows that most cardiac patients are motivated to quit or are already in action at their hospital stay. The overview shows in congruence with the information derived from the general behavioral explanation models that attitude, self-efficacy, and a supportive environment are important determinants of cessation among the target population. The study by Kristeller (1992) suggests that the importance of the psycho-social factors differs with the phases. Therefore, the intervention should make it possible to tailor the supplying of attitude, selfefficacy enhancing information and social support information to a patient's phase of change. Furthermore, the manifestation of the disease itself and nicotine dependency need to be emphasized. The other more distal determinants reported for CHD patients are probably less important for the intervention development, since they are more 
difficult to change by health education interventions than the proxinal determinants (Conner \& Norman, 1996; De Vries \& Mudde, 1998).

The analysis of determinants that was derived from literature and described in this subsection formed the basis of the development of the intervention. Unifortunately, an empirical analysis of the intentions and deteminants of the target group prior to the development of the intervention was not possible within the time frame of the present project The main reason was that the effectiveness study reported in this thesis started one and al hal months after I became involved in the project. At the time when I became involved, the development of the intervention was already in progress and had almost been finished. Because the existing literature did not provide suffictent information on the psycho-social determinants related to the various phases of change in cardiac inpatients, my project also included an empirical study of the phases and underlying determinants of this specific target group. This study is described in chapter two of this thesis. Although the outcomes of this study could not be used in the development of the intervention, it was felt that they could be helpful in improving existing interventions and could contribute to the theoretical insights into the process of smoking cessation and its underlying determinants.

\section{Determinants of smoking cessation assistance by intermedianies}

A previous subsection showed that the smoking cessation assistance of the inter mediaries is not satisfactory. This part aims to provide insight into the uriderlying reasons in order to facilitate the process of implementation of a smoking cessation intervention.

In general, physicians working in hospitals consider it their task to pay attention to their patients' smoking behavior and to advise them to quit smoking, but they are pessimistic about the effects of their efforts (Cummings et al., 1989; Duncan et al., 1991; Frank \& Kunovich-Frieze, 1995; NIPO, 1995; Wells, Lewis, Leake, Schleiter, \& Brook, 4986; Wiebing \& Baan, 1997). One Dutch study, however, reported that most physicians $(72 \%)$ do not consider it their responsibility to persuade patients to quit smoking (Dekker, 1990). This study also reported that most physicians believed that patients would quit if they advised them to do so. Task perception and attitude seemed to be negatively related to the physicians' own smoking behavior (Frank \& KunovichFrieze, 1995; Miwa, Fujita, Miyagi, Inoue, \& Sasavama, 1995; Tessier, Thomas, Nejari, Belougne, \& Freour, 1995; Waakens et al. 1992; Wiebing \& Baan, 1997). Physicians who smoked perceived their own smoking behavior as a barrier to providing smoking cessation assistance (Frank \& Kunovich-Fileze, 1995; Wiebing \& Baan, 1997). Some studies reported that physicians, and more specifically cardiologists, saw themselves as lacking sufficient skills and knowledge for the provision of smoking cessation care (ressier et al., 1995; Waakens et al, 1992).

Nurses also found it their responsibility to assist patients to stop smoking (Faulkner \& Ward, 1983; Goldstein et al. 1987; Nagle et al., 1999; NIPO, 1995; Wiebing \& Baan, 1997). This was, however, restricted to patients who were suffering from a smo- 
king-related disease and were willing to quit. It was also found that nurses were not convinced of the effects of their efforts (Sanders et al., 1986; Wiebing \& Baan, 1997). In several studies, it was reported that nurses have insufficient knowledge of effective smoking cessation strategies and lack the necessary interparsonal skills (Faulkner \& Ward, 1983; Lilington 1997; Nagle et al. 1999), while one study reponted that Dutch nurses did not perceive notable barriers with respect to the provision of smoking cessation care (Wiebing \& Baan, 1997). Contrary findings have also been ieported with respect to the influence of nurses own smoking behavior on their willingness to prowde smoking cessation care. Some studies reported that being a smoker was negatively associated with pro smoking cessation care beliefs (Adriaanse, Van Reek, Zandbelt \& Evers, 1991; Dore \& Hoey, 1988; Goldstein et al, 1987), while another study found this to be helpful in assisting patients to stop smoking (Nagle et al., 1999). Nagle and colleagues suggested that nurses have to be trained in the provision of smoking cessation care and need to be supported by hospital staff and supportive hospital policies, such as extra time and incentives.

In order to enable the nurses and cardiologists to perform their role successfully, the following conclusions can be drawn. Both intermediaries need to develop skills in assisting patients to stop smoking. For this purpose, it is also necessary that they have sufficient knowledge about the process of behavioral change in smoking cessation and how this process can be facilitated. They furthermore have to be convinced of the positive effects of their efforts, and attention has to be paid to the effect their own smoking behavior can have on the way they approach their patients.

\section{Access Point Analysis.}

The final step of the problem analysis is to assess how the target group can best be reached and which intervention methods patients and intermediaries prefer. As alsea dy indicated, the target group were hospitalized CHD patients who smoke. The period of hospitalization was considered to provide a good opportunity for smoking cessation interventions. Not only because it allows a large part of the potential target group to be reached, but also because patients experience personal walnerability to the dangers of smoking at that moment (Emmons \& Goldstein, 1992). Hence, quitting motivation and receptiveness to smoking intenventions may be at their peak. Another argument to intervene during the hospital stay was the fact that activities could be easily embedded in the existing health care activitien. A final argument was that all patients visited the cardiology outpatient clinc for a check-up after discharge, allowing follow-up care. The previous subsection showed that the potential contributions of nurses and cardiologists has been underutilized until now.

In the Netherlands, CHD patients can in general be admitted to three types of unit.. Patients suffering from acute CHD symptoms (e.g. MI) are admitted in emergency to an 
intensive coronary care unit ( $\mathrm{CCU}$ ), and move to a medium (MCU) or low care cardiology unit (ICU) as their condition stabilizes. These units can, however, be orated within the same ward. Patients who are scheduled for a specific treatment (e.g., CABG or PTCA) often stay for only a few hours in a CCU and are subsequently moved to a MCU or LCU, depending on the stability of their heart condition. Because this structure differs in different hospitals, and since we wanted to reach all smoking CHD patients, it was decided not to exclude any type of unit. Thus, the intervention could start at the $\mathrm{CCU}$ and could be continued at the MCU or LCU, but could also only take place at a MCU or LCU.

Cardiologists and nurses were considered to be appropriate intermediaries. Patients tend to regard the cardiologist as an authority and a credible source (Burling, Singleton, Bigelow, Baile, \& Gottlieb, 1984; Fowler, 1997; Hollis, Lichtenstein, Mount, Vogt, \& Stevens, 1991). In addition, nurses have the greatest frequency and duration of contact with patients, are a continuous factor in hospital care and have a recognized role in providing patient education (DeMello, Hoffman, Wesmiller, \& Zullo, 1989; Nagle et al., 1999). Co-operation between health care providers has been shown to increase the effectiveness of a smoking cessation intervention (Fiore, 1997; Kottke, Battista, Defriese, \& Brekke, 1988; U.S. Department for Health and Human Services [U.S. DHHS], 1996; Wetter et al., 1998). Moreover ${ }_{\text {i }}$ co-operation between nurses and cardiologists reduces the burden for the latter (Hollis et al., 1991) and studies among cardiac inpatients (Burling et al., 1984; Orleans et al, 1990) have revealed that a personal stopsmoking advice from a hospital physician had made an unique contribution to patients' quitting behavior and was considered a motivational force for quitting.

As has been suggested by several researchers (Ockene et al., 1992; Orleans et al., 1990; Rosal et al. 1998; Sirota, Curran, \& Habif, 1985; Strecher, Becker, Kirscht, Eraker, \& Graham-Tomasi, 1985), another option might have been to use special intervention teams like counseling psychologists or smoking cessation specialists to carry out the intervention. However, in anticipation of future dissemination, it was deemed to be better to link up with the existing ward situation, with nurses and cardiologists delivering the intervention.

Only one study has been published on the preferences of cardiac inpatients concerning smoking cessation assistance. This study reported that there was little interest in formal treatment options among cardiac inpatients. Quitting without help was the most preferred cessation strategy, followed by, in order of descending popularity, the use of self-help guides and counseling by physicians (Emmons \& Goldstein, 1992). Nowadays, it is, however, presumable that nicotine replacement therapy or medical treatment with Zyban (which is currently registered as a medication for smoking cessation) is also popular.

With respect to the preferences and possibilities among the intermediaries, we obtained qualitative information during our meetings with a working group on cardiovascular nursing, who took part in the project group. The working group stressed the importance of a minimal-contact approach, because they only could spend a maximum of 15.30 minutes for smoking cessation assistance. They also stated that a smoking 
cessation intervention had to be flexible to use and should be easy to embed within the existing routine and infrastructure. The preferences of cardiologists were not as sessed explictly, although indications were obtained from the nurses of the working group and from a qualitative study among a large sample of Duch general practitioners, which indicated that physicians could spend three minutes on smoking cessation assistance (Boekema, Kooi, Seydel, \& Pieterse, 1990; Pieterse, 1999). This study also indicated that an intervention should delegate certain tasks to a practice assistant, should not interfere with routine medical practice and should require a minimum of organizational changes.

\section{Behavioral Change}

The main scope of this phase is to formulate goals and objectives, to translate them into practical methods and to find ways to assess the effectiveness of the intervention developed.

\section{Setting goals and objectives.}

No general program goals were formulated in the present project, since the imme. diate health effects could not be assessed during the time of the effectiveness study. Moreover, the previously described problem amalysis revealed a body of evidence that smoking cessation following a cardiac event has almost immediate health benefits, including decreased mortality and morbidity. The intervention primarily aimed at tertiary prevention, which concerns preventing the further development of an existing disease, minimizing its severity and optimalizing care (Green \& Kreuter, 1999; Tones \& Tilford, 1994). Because smoking is also associated with the development of diseases such as (lung) cancer and chronic obstructive pulmonary diseases, the intervention can also have a primary prevention impact on the occurrence of these diseases.

smoking cessation is the ultimate behavioral change objective of the intervention. objectives might also relate to the progression through the phases of behavioral change. Precontemplators, for example, are not ready for action and a reasonable goal for them

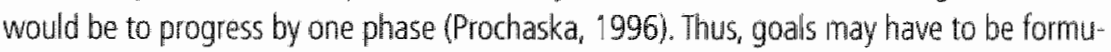
lated separately for each phase. Because previous research had shown that the target group manly consisted of smokers in the contemplation or action phases (Kristeller et al. 1992; Ockene et al., 1992), phase transition goals had a low priority in this project. Within the scope of smoking cessation, the main behavioral outcome was point preva lence abstinence (not having smoked in the seven days prior to the measurement) after three months. Additional outcomes were continuous abstinence after three months, and point prevalence abstinence and contimuous abstinence after twelve months. Con- 
tinuous abstinence was regarded as a secondary ouicome because it has the disadvantage that only a small minority of smokers actually change so radically from smoking to non-smoking without lapses or relapse, while it also excludes smokers who take delayed action to quil (Velicer, Prochaska, Rossi, \& Snow, 1992). Measurements of cessation after welve months were considered to be useful to examine whether minimal aftercare efforts could achieve long term effects.

The behavioral change objective formulated for the purpose of the present project presumed a $50 \%$ point prevalence abstinence rate among patients exposed to the intervention, compared to a $40 \%$ point prevalence abstinence rate among the patients who were given routine care (i.e., no systematic attention to smoking behavior). Rates concerning the additional outcomes were not explicitly specified. The estimates in the behawioral change objective were based on previous studies. Although the literature reported an average of $45 \%$ spontaneous quitters among CHD patients (Deckers et al. 1994), we estimated that only $40 \%$ of our patients would quit without help, because the studies reported in literature mainly concermed patients who were hospitalized for a first M, while our intervention also targeted people with less severe CHD and a longer history of CHD, whose spontaneous quitting rate is lower (Ockene et al., 1992; Rigotti el al., 1991). Based on earlier effect studies among cardiac inpatients (Ockene et al., 1992; Orleans et al. 1990; Sirota et al., 1985; Strecher et al., 1985; Taylor et al," 1990) and a review of smoking cessation interventions in medical practice (Kottke et al. 1988), we estimated that a minimal-contact intervention combined with self-help guidance could result in an additional 10\% quitters. The studies mentioned here concerned, however, intenser interventions. Effects of minimal-contact interventions for cardiac inpatients were not reported in literature so far.

\section{Program development.}

This subsection reports on the guidelines and the theoretical framework that formed the basis of the smoking cessation intervention, followed by a description of the intervention.

Guidelines for the development of the intervention

First, the outcomes of the problem analysis provided useful guidelines for the development of the intervention. The literature suggested that patients preferred self-help guidance and minimal counseling. Nurses and cardiologists were found to be adequate sources for the provision of a smoking cessation intervention, and their co-operation was suggested to increase the effectiveness of an intervention. Furthermore, the working group on cardiovascular nursing indicated that a minimal approach was necessary because of the heavy workload and pressure of time at the cardiology wards. Therefore, a minimal approach, in which a mimimal amount of counseling would be combined with self-help, was considered to be an appropriate method. The analysis of determinants indicated that the intervention would have to include efforts to tailor the smoking cessation assistance to the patients" phases of change. 
Second, published guidelines on hospital-based smoking cessanion interventions (Orleans, Kristeller, \& Gritz, 1993b; Orleans \& Ockene, 1993; Orleans et al. 1990) revealed that a practical and powerful intervention comprises a brief stop-smoking advce from a physician, combined with stage-tailored nurse counseling, follow-up care after hospital discharge and the provision of self-help materials. In these guidelines, it was also recommended to combine the provision of motivational information with a short training to teach patients how to cope with high risk situations.

Thind, the Health Counseling model (Gerards, 1993; Gerards, 1997;" Gerards \& Hospers, 1991) provided useful information for the development of the intervention. This model is based on theories of behavioral change, takes the phases of change into account and emphasizes the patient's own responsibility for behavioral change. An application of the HC model, the so-called Minimal intervention Strategy (MIS) was tested on its efficacy and feasibility in a large number of general practices in the Netherlands and was found to result in an additional cessation rate of $8.5 \%$ compared to usual care (Pieterse, Seydel, Mudde, \& De Vries, 1994).

Because the MIS was effective, met the intervention preconditions derived from the problem analysis, and was in line with the guidelines described earlier in this subsection, the Health Counseling model and its MIS application served as the framework for our intervention. The model distinguishes three phases: preparation, implementaw tion and aftercare (see Table 1.2). Preparation is meant to make patients aware of the problem and the need for behavioral change, and to motivate them to change. The second phase includes the behavioral change itself. In this phase, the health care worker urges the patient to change his behavior by setting targets for him to meet and by giving behavioral instructions. The final phase concerns aftercare and is meant to achieve behavioral maintenance and to prevent relapse.

Table 1.2 shows that each phase consists of several steps, which correspond to the phases of behavioral change (Prochaska et al., 1992). The preparation phase mainly focuses on precontemplators who have to become aware of the health problem and its relationship with their behavior, and have to become motivated to change. Contemplators and preparators are already motivated to change and have to be prepared for this behavioral change, which is the locus of the next phase of the HC model. People who are already in action (actors) need help in sustaining their new behavior; this is addressed in the aftercare. Depending on a patient's phase of change, health care prowiders have to go through the different phases to guide his behavioral change.

The preparation phase is based on earlier described social cognitive theories like Ajzen's Theory of Planned Behavior (Ajzen, 1991), the ASE model (De Vries et al., 1988; De Vries \& Mudde, 1998) and Bandura's Social Cognitive Theory (Bandura, 1986). AC. tions in the implementation phase are derived from the goal-setting theory (Locke \& Latham, 1990; Locke \& Latham, 1994) and from what is known about the positive behavioral effects of making public commitment (Kiesler, 1971). The goal-setting theory assumes that setting specific challenging goals (e.g. in terms of behavioral change) 


\section{Table 1.2 Health Counceling model (translated from Gerards 1993 )}

\begin{tabular}{|c|c|c|}
\hline $\begin{array}{l}\text { phase } \\
\text { preparation }\end{array}$ & $\begin{array}{l}\text { steps } \\
\text { ceate awareness }\end{array}$ & $\begin{array}{l}\text { goal } \\
\text { help patients to get insing into } \\
\text { the relationshu between their } \\
\text { disease or symptoms, recovery } \\
\text { and their behavion }\end{array}$ \\
\hline
\end{tabular}

\section{weigh up motwate patients to hange pros and cons \\ the pros and cons their behavion}

decision making support patients to make the decision to change

detect and remove barriers feg. emotions, willoower, selfefficary:

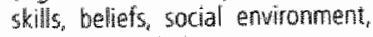
practical possibilities, urges or cravings?

behavioral change change the behawor

counsel patients curing the change

-provide behaviloral instuctions -sel langets

- prowide supportive materials use principles of operant conditioning teach patients about self control

attercare

Lyehavior mantainance guide patient to maintain the desired behavior

-feedback 10

detect high-risk situations

-help patient to acquire coping skills change hestyle

relapse prevention

teach patient how to cope with lapses or relapse assess the way the patient attributes failures and if needed, try to change the way of attribution

improves task performance. Aftercare is based on the theory of relapse prevention (RP) by Marlatt and Gordon ('Marlatt \& Gordon, 1985). RP is a cognitive behavioral approach in which an individual learns to cope with high-risk situations in order to prevent lapses and relapse into the undesired behavior.

Intervention for cardiac inpatients (C-MS)

The following protocol was developed for the project (see Figure 1.4). First, the cardiologist advised patients to stop smoking. This step was added to the MIS because conclusive reviews stated this to be essential for cardiac patients (Burling et al., 1984: Fiore, 1997; Fowler, 1997; Miller, 1993; Orleans \& Ockene, 1993; Orleans et al., 1990; Raw, McNeill, \& West, 1999). Theoretical underpinnings for this step are that the smoking risk becomes personalized, the relationship between (progression of) the disease and the patient's own behavior becomes clear, and the desired behavior is clearly stated (Gerards, 1997; Mesters, Creers, \& Gerards, in press; Orleans et al., 1993b). Moreover, it was assumed to lend additional authority to nurses to intervene on the patients" smoking behavior. The second step was carried out by nurses and concerned the assessment of patients' smoking behavior, their level of addiction and their motivation to quit ("smoker profile"). Thirdly, nurses tried to motivate patients with a low motivation to quit by addressing the positive consequences of smoking cessation. If 
patients were already motivated to quit or already had decided not to smoke anymore. nurses omitted this step. The next step concemed the assessment and ellmination of barriers that might hold patients from quitting. This step was also carried out by nurses and included an assessment of high-risk situations that patients expected during the quitting process. The nurse stimulated the patient to prepare for highwrisk situations by suggesting ways of coping with them. This step was also carried out in patients who already had decided not to smoke anymore. In the fifth step, the nurse tried to agree behavioral change targets with the patients. The nurse stimulated patients to set a quit date, which was written down in the presence of the patients. Also if a patient already had decided not to smoke anymore, he or she was stimulated to make the quit day (which was obviously a few days earlier) official by writing it down. The final step concerned the aftercare and included 1) a telephone call by the nurse two weeks after discharge (this was optional since patients were asked whether they wanted to be called), and 2) for every patient personal attention and support for not smoking by the cardiologist four to six weeks after discharge. In addition, general practitioners were asked to address the patient's smoking behavior if the patient would visit the general practice after discharge. The timing of the telephone call was chosen because the risk of relapse is considered to be highest within three weeks after quitting (Zhu et al. 1996). Several studies among cardiac inpatients using telephone follow-up in addition to inhospital counseling, suggested its, usefulness, although none of these studies isolated the effects of telephone counseling from those of other intervention components (DeBusk et al., 1994; Ockene et al. 1992; Taylor et al., 1990). From theoretical point of view, a telephone call is useful because it is follow-up support in which feedback, reinforcement, and facilitation (principles of education) can be provided (Bandura, 1986; Locke \& Latham, 1990; Mullen, Mains, \& Velez, 1992). The cardiologist re assessed the patients' smoking behavior in order to prevent relapse, or if patients had already relapsed, to motivate them to make another quit attempt. This was embeddied in the standard outpatient check-up by the cardiologist.

In addition, nurses had to provide patients with written materials: a self-help manual and a brochure. The brochure focused on the dangers of smoking for people suffering from CVD. The self-help manual was written for the general Dutch population and had proved to be a useful instrument in several Dutch multicomponent smoking cessation programs (Mudde, de Vries, \& Strecher, 1996; Mudde, Willemsen, \& Van Assema, 1994; Willemsen \& De Vries, 1995; Willemsen, De Vries, Van Breukelen, \& Genders, 1998). The content of this guide was structured in the same way as the protocol. Nurses could decide for themselves when the best time was to administer the materials. Furthermore, in cases with more severe nicotine addiction or if patients preferred a more intensive treatment, nurses pointed out to them the possibility of participating in a smoking cessation group program in their own neighborhood after hospital discharge. In contrast to the MIS our protocol did not include nicotine replacement therapy (NRT) as an aid for patients with withdrawal symptoms, because it was thought to have 
adverse cardiovascular effects, particularly if patients continue smoking (Benowitz \& Gourlay, 1997; Gourlay \& Benowitz, 1996; U.5. DHHS, 1996). At the time of preparing the present thesis (2001). NRT is judged to be safe, though should be used with caution among particular cardiovascular patient groups (Fiore, Balily, Cohen, \& et al., 2000).

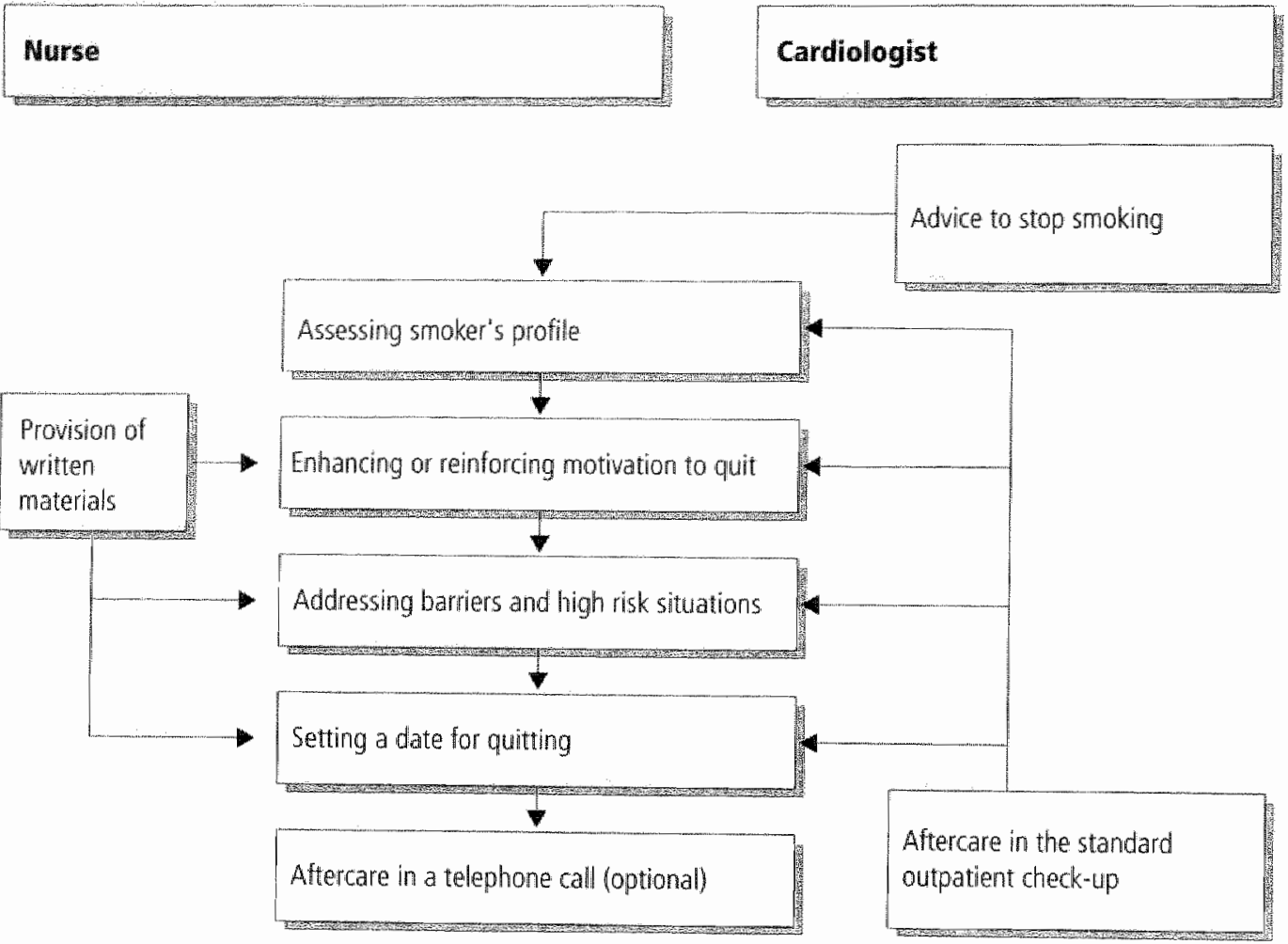

Eloure 1.4 C-MS protocol.

Because the problem analysis had shown that nurses had insufficient knowledge and skills to assisit patients to quit smoking, a nurse training was incorporated. All nurses who had to work with the protocol received a two-hour training, which was initially structured according to a train-the-trainer principle. This meant that two nurse-representatives of each ward (called internal program co-ordinators (IPCS) from now on) were trained by staff members of the Dutch Foundation an Smoking and Health (Stivoro) and by the researcher. Subsequently, the IPCs trained their colleagues. Furthermore, a poster with a description of the protocol was sent to each ward, together with a reference book including instructions on how to apply the different steps in the protocol. The trained IPCS also served as back-ups to whom fellow nurses could go 
when they had problems or questions relating to the protocol. In addition, these representatives had the task to inform and instruct the cardiologists about his task. In addition to verbal instructions, they provided the cardiologist with a self-explanatory flowchart of the protocol, and a letter which included an explanation of the protocol and a task description. To remind the cardiologist during the aftercare period, the patient file was stamped with a prompt and included a short report on the content of the in-hospital part of the intervention.

\section{Program evaluation.}

The main goal of the present project was to evaluate the effectiveness of the $C$ MIS, since the original intervention (MIS) had already been tested for its efficacy (Pieterse, 1999). The evaluation study initially started as a randomized controlled trial, which took place from November 1995 till June 1998. Randomization was not succes. sful, however, since two hospitals ultimately indicated to want to serve only as controls, while two other hospitals onlly wanted to co-operate if they were included in the experimental group, which makes it more appropriate to describe this a Pretest-Posttest quasi-experimental study ex post (Rossi \& Freeman, 1993). As already indicated in a previous subsection, the main goal of the study was to study the effects of the inter vention on point prevalence abstinence after three months.

Power analysis revealed that a sample of 650 patients was necessary to detect a difference of $10 \%$ between control and experimental patients after three months. This sample size would provide a $80 \%$ of power $(p<.05$, one tailled). Assuming a $20 \%$ dropout rate, the sample thus needed to include 815 smoking patients at baseline. For detailed information on the effectiveness study, the reader is referred to chapters three and four, which report on the intervention effects after three and welve months.

Because utilization, degree and quality of implementation are assumed to influen. ce the effectiveness of an intervention (MacDonald, Veen. \& Tones, 1996; Nutbeam. 1998; Sussman, Dent, Stacy, Sun, \& et al, 1993; Tones, 1997; Windsor, Baranowski, Clark, \& Cutter, 1994), process measurements were conducted at the three-months posttest, as described in chapter three.

\section{Continuation}

Because the existence of an effective program does not automatically imply largescale usage, dissemination is am important issue in the program planning. The likeli. hood of successful dissemination can be increased by establishing intersectoral collaboration and support, by developing diffusion strategies and by developing supportive policies. It has been recommended to anticipate on these issues right at the 
beginning of a project. Activities employed in this project to promote continuation the final phase in the diffusion process: are addressed below.

\section{Intersectoral collaboration.}

The likelihood of continued use of an intervention will be increased if stakeholders from all areas within the domain of the intervention have participated in the development and its implementation. As mentioned earlier, the linkage approach can be very useful for this purpose. The establishment of a linkage group enables stakeholders to participate in the process of program preparation, testing and diffusion. Representatives of various groups are needed in the linkage group because of their distinctive functions. Representatives of the research group are needed because of their responsibility for developing the intervention and conducting the program evaluation; the resource group needs to be represented for the provision and development of program materials; representatives of the intermediary group are needed to ensure that the program will be feasible in daily practice; persons representing the target group are needed to allow treatment preferences to be taken into account; a finance group needs to be represented to monitor the intervention and to make sure that it does not conflict with their overall mission. Besides, a support group may be represented by persons who might be able to provide support for the project (e.g. by their expertise or network) (De Vries, 1998).

The linkage group in the project described in this thesis started two years before the effectiveness study actually took place. This group (see Figure 1.5) included representatives of the research, finance and resource groups and the group of intermediaries. The support group was represented by a researcher from Twente University who had developed and evaluated the MIS. Unfortunately, the linkage group did not include representatives of cardiac inpatients nor of cardiologists. لlust before and during the effectiveness study, the linkage group was supplemented by IPCs from the hospitals who participated in that study. During the whole project, the linkage group had scheduled meetings every two months in order to momitor the development of the interven tion, the testing and the process of collaboration. 


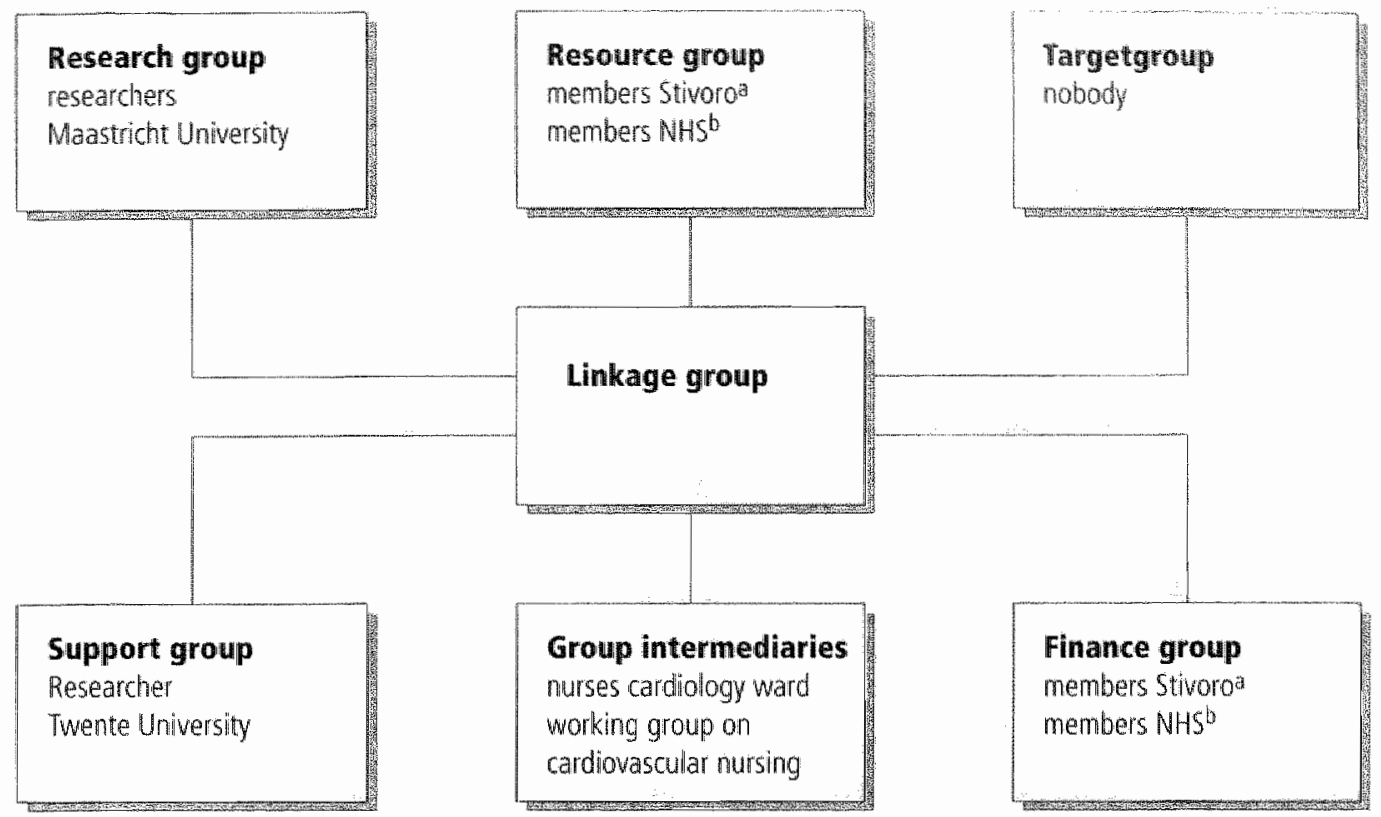

Figure 1.5. Linkage group applied to the project.

Note. "Dutch foundation on Smoking and Health, "Netherlands Heart Foundation

\section{Diffusion strategies.}

Besides the need for a linkage group, it is also necessary to obtain insight into factors that stimulate and hinder adoption, implementation and continuation of an intervention. This information is necessary to formulate strategies for future diffusion.

Applied to the project described in this thesis, Roger's diffusion theory (Rogers, 1995) suggests that a first step has to be to achieve awareness among nurses of the existence of a new protocol. Second, it is necessary to achieve adoption, also referred to as commitment or intention to try out an innovation. The third step concerns the actual implementation of the new protocol. Finally, the protocol has to become embedded in daily practice, which is referred to as institutionalization or continuation.

Although this project primarily concerned the effectiveness of a smoking cessation intervention and diffusion was beyond its scope, one pilot study was conducted on the intentions and determinants of continued use of the intervention by ward nurses. This study, which is described in chapter five of this thesis, was carried out among nurses who had worked with the protocol during the effectiveness study and was conducted immediately after the interventions in the experimental group had been finished. The 
goal of this study was to assess the differences between nurses who were willing to continue working with the smoking cessation program and those who were not. This information was expected to help us identify possible problems and barriers that were perceived by nurses concerning the continuation of the intervention.

\section{Supportive strategies.}

To achieve successful adoption, implementation and institutionalization of a hospital-based smoking cessation intervention, the mere provision of a protacal may not be enough. Supportive policies at the micro, meso and macro levels may be needed to facilitate diffusion in a broader context. Policies at the cardiology wards (the micro level) have to support the integration of smoking cessation care within the overall care. Nurses, for example, must be allowed sufficient time to counsel patients on their smoking behavior and to follow a training in protocol usage. The provision of (tinancia) incentives may also support nurses decisions to provide smoking cessation care. At the hospital (or meso) lewel, supportive action needs to be taken, e.g "by non-smoking ward policies and smoking restrictions for the health care providers themselves during working hours.

During the project, Stivoro, one of whose major activities is the implementation and continuation of smoking cessation interventions in the Netherlands, initiated strategies at the micro, meso and macro levels to support further diffusion and continued use of the C-MIS. For example, the organization was developing a nationwide campaign to introduce the intervention in all Dutch hospitals, was negotiating with the Dutch department of public health, well-being and sports to obtain financial resources for this purpose and was making efforts to get Dutch hospitals to adhere to the Dutch smoking prohibition law for public places. A further step may be the mandatory instalIment of smoking cessation support for hospitalized CHD patients who smoke.

The issues addressed above illustrate that chances of further diffusion and continued use cannot be judged in a vacuum and depend on contextual factors. Policy development and changes in current practices are assumed to take a long time, while the scarce resources and time restraints in the project described in this thesis made it impossible to intervene actively in this respect. 


\section{PSYCHO-SOCIAL DETERMINANTS AND MOTIVATIONAL PHASES IN SMOKING BEHAVIOR OF CARDIAC INPATIENTS*}

\section{Abstract}

Background. To explore passibilities for health education, this study analyzed the differences in motives regarding smoking cessation of cardiac inpatients in various motivational phases. The Attitude - Social influence - Self Efficacy Madel (ASE model) was used to assess motives, while motivational phases were measured with concepts from the Transtheoretical Model.

Methods. Data on smoking behavior, attitudes, social influences, self-efficacy expectations, and motivational phases were collected from a sample of 532 cardiac inpa tients. A revised typology of the Transtheoretical Model was used for measuring motivational phases and resulted in four groups: smokers in precontemplation, smokers in contemplation, externally motivated actors, and internally motivated actors. Analysis of variance with Tukey's multiple comparison test was used to study differen. ces in psycho-social determinants between these four groups.

Resuls. As hypothesized, externally motivated actors differed from internally motiwated actors in having less positive attitudes, less sacial support, and lower self-efficaw cy expectations. Attitudes and social support were most positive among smokers in contemplation and internally motivated actors. Self-efficacy expectations were lowest among subjects in precontemplation and contemplation.

Conclusions. Subjects in different motivational phases differed in their psycho social determinants. Therefore, it is recommended that stage-tailored education will be developed. Finaly, externally motivated actors have to be approached in a different way than internally motivated actors.

* Slightly different version published as: Bolman, C. \& De Vries, H. 1998). Psychomsocial determinants and motivational phases in smoking behavior of cardiac inpatients. Preventive Medicine, 27, 738-747. 


\section{Introduction}

It has been known for a long time that cigarette smoking is one of the major risk factors for coronary heart diseases (Havik \& Maeland, 1988). Smokers show a 70\% excess rate of mortality from coronary heart diseases and they are considered to have a two- to fourfold increased risk of developing coronary heart disease (U.S. DHHS, 1983).

People who quit smoking after myocardial infarction or other coronary heart diseases have much better prognoses than people who persist in their smoking behavior. According to a recent meta-analysis (Deckers et al., 1994), smoking cessation in patients with newly established coronary artery disease reduces mortality after 1 year by almost 50\%. Furthermore, smoking cessation after the first myocardial infarction reduces the risk of recurrence by half (Mukahy, 1983). Smoking cessation can improve the prognosis of cardiac patients more than any other treatment wDeckers et al., 1994; U.S. DHHS, 1990). Despite these promising efferts of smoking cessation on morbidity and mortality, 20 to $60 \%$ of cardiac inpatients persist in their smoking behavior or relapse after hospital discharge (Deckers et al., 1994; Ockene et al., 1992; Orleans et al., 1990; Rose \& Colwell, 1992; Taylor et al., 1990). The development of effective education strategies to motivate cardiac inpatients to quit smoking requires knowledge of their motives for not quitting smoking (Green \& Kreuter, 1991).

The present study used the ASE model of behavioral determinants (De Vries et al. 1988; Kok et al., 1991) to obtain more insight into the motives of smoking cardiac inpatients by comparing them with those who have quit. This model has proved to be very useful in understanding the behavioral intentions of various populations concerning smoking cessation rDe Vries \& Backbier, 1994; Mudde, Kok, \& Strecher, 1995b; Willemsen et al., 1996). The ASE model originated in the Theory of Reasoned Action (Fishbein \& Ajzen, 1975), and Bandura's Social Cognitive Theory (Bandura, 1986). It states. (see Figure 2.1) that behavior can be explained by behavioral intention, which in turn is determined by attitudes, perceived social influences, and self-efficacy expectations (the so-called psycho-social determinants). External variables, such as sociodemographic factors, are assumed to influence behavior through the three psycho-social determinants and behavioral intention. A person's attitude is determined by the balance between expected positive and negative consequences of the behavior in question (the so-called pros and cons) (De Wries \& Backbier. 1994). With respect to social influences, three independent influences are assumed to play a role (De Vries et al., 1995). First, the perceived norms of impontant others concerning the behavior. Second, the support or pressure a person experiences from his direct environment. The third social influence appears when the behavior of important others is taken as an example (modeling) (Bandura, 1986). Self-efficacy refers to a person's expectations regarding his or her capability to perform the desired behavior. The ASE model is comparable with Ajzen's Theory of Planned Behavior (Ajzen, 1991), but differs 
in the way psycho-social deteminants are measured. For example, the ASE model distinguishes three types of sociall influences -social noms, perceived behavior of others, and direct support to perform a particular behavior-while the Theory of Planned Beha. vior only measures social norms. For more details see De vries and Mudde (1998).

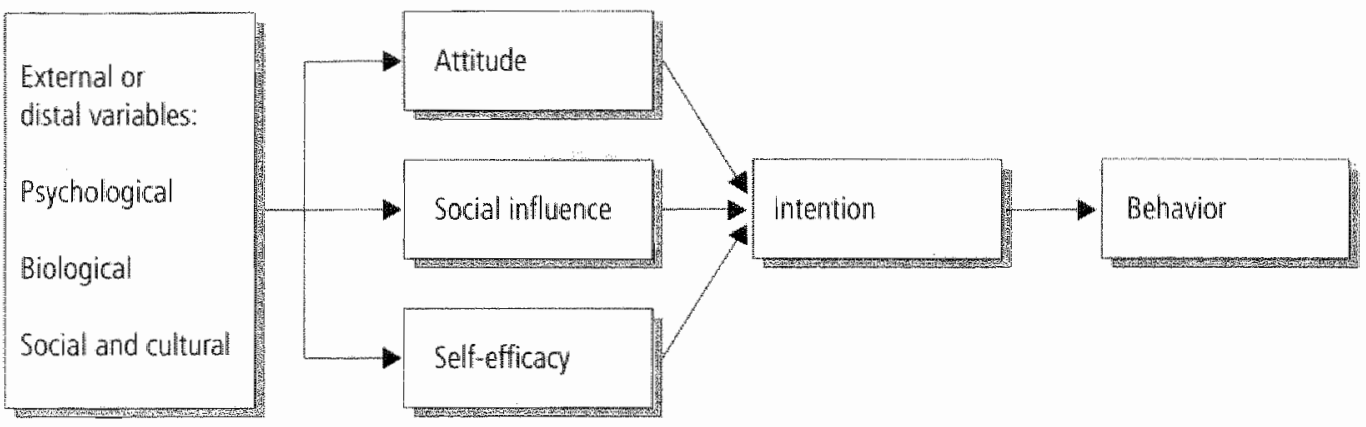

Figure 2.1. ASE model (adapted from De Vries : Mudde, 1998)

Recently, smoking cessation intentions and simoking behavior have often been measured with the so-called stage constructs of the Transtheoretical Model (TM) deven loped by Prochaska and DiClemente (Prochaska \& DiClemente, 1992; Prochaska et al., 1992). This model states that smoking cessation is a process by which smokers move from precontemplation (not considering behavioral change) to contemplation (considering behavioral change within half a year) and preparation (planning to change the behavior within 1 month), belore engaging in a quit attempt (action). With respect to smoking cessation among cardiar patients, the study of Kristeller (1992) confirmed the validity of this concept. The present study used the ideas of TM for measuring intentions and behavior, although some adjustments to the stage constructs were necessary for the present population, since not all cardiac inpatients engaged in quitting voluntarily; they could be "forced" into action due to hospital regulations'. Hence, a distinction should be made between externally and internally motivated actors. Moreover, we prefer to speak of precontemplation and contemplation phases, since the stage concept refers to psychological develapmental stages. Consequently, four groups were studied: smokers in the precontemplation phase, smokers in the contemplation phase, externaly motivated actors, and internally motivated actors.

Earlier Dutch research integrating the ASE model with the stage concept of the TM showed that smokers in the precontemplation phase could be distinguished from actors and smokers in the contemplation phase by their negative attitudes toward quitting and their lower perception of positive consequences of the healthy behavior. Moreover, the latter group were found to perceive more social support for engaging in the healthy behavior than smokers in the precontemplation phase. Self-efficacy was found to be low for smokers in the precontemplation phase and snokers in the con- 
templation phase, but was significantly higher among actors (De Vries \& Backbier, 1994). The resulting 0 pattern depicted in Figure 2.2, was replicated in four other Dutch studies on smoking cessation (De Wries et al, 1998) and in one study on fat intake (Brug \& Van Assema, 1995). Based on this pattem, several researchers (Brug, Hospers, \& Kok, 1997; De Vries \& Backbier, 1994; Dijkstra et al., 1996) suggested that people in different motivational phases need different types of information to stimulate transition through the phases.

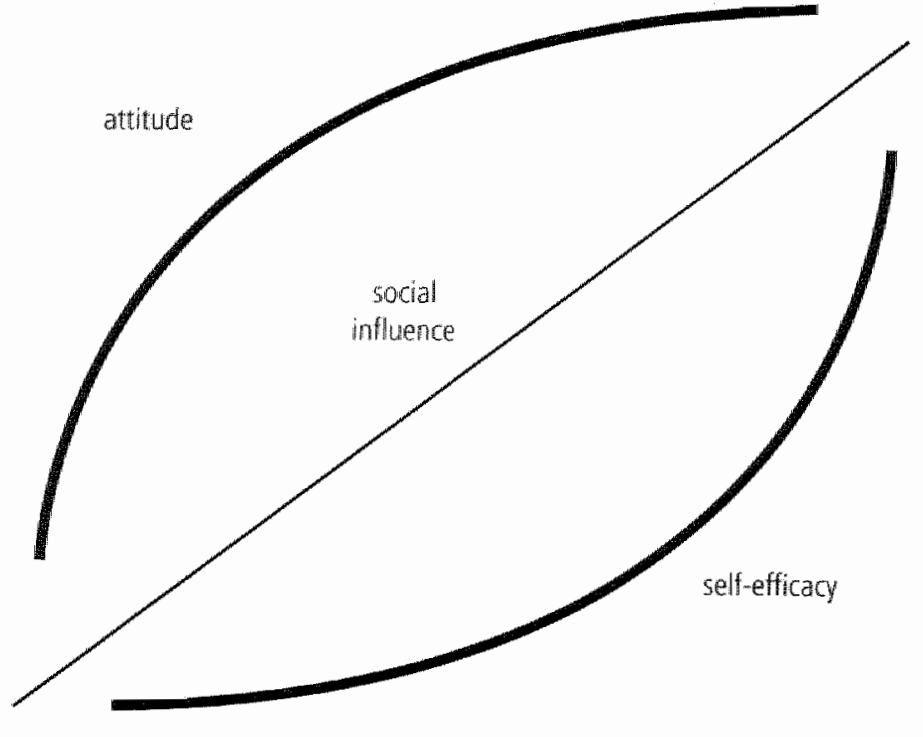

prewcontemplation

contemplation

preparation

action

Eigura 1.3. The pattern

The first goal of the present study was to assess whether externally and internally motivated actors differed with respect to their psycho-social determinants. It was hypothesized that externally motivated actors would have weaker intentions to remain nonsmokers and would perceive significantly fewer advantages and more disadvantages of nonsmoking. Furthermore, they were expected to experience less social support and to show lower levels of self-efficacy than internaly motivated actors (hypothesis 1). The second goal was to compare the population of smoking cardiac inpatients in different motivational phases with regard to their attitudes, social influences experienced and perceived self-efficacy. On the basis of earlier research (Breteler et al. 1988; De Vries \& Backbier, 1994; De Wries et al., 1998; Dijkstra et al., 1996; Prochaska \& DiClemente, 1992) it was hypothesized that smokers in precontemplation would differ 
significantly from those in contemplation and in internally motivated action by having significantly lower perceptions of the advantages of nonsmoking (hypothesis 2). Furthermore, it was expected that smokers in precontemplation would encounter significantly less social support toward nonsmoking than internally motivated actors (hypothesis 3). With respect to self-efficacy expectations, it was expected that internally motivated actors would have significantly higher levels of self-efficacy expectations than smokers in precontemplation, smokers in contemplation, and externally motivated actors (hypothesis 4). The third goal was to analyze whether the previously detected $\varnothing$ pattern could be replicated for cardiac inpatients. It was hypothesized that the $\varnothing$ pattern could be replicated for the sample consisting of smokers in precontemplation, smokers in contemplation, and internally motivated actors, but not for the externally motivated quitters since they would feel forced into action and therefore would have different motivational characteristics than internally motivated actors (hypothesis 5).

Since smoking cessation can improve the prognosis of cardiac patients more than any other treatment (Deckers et al., 1994; U.S. DHHS, 1990), it was important to obtain insight into motivational determinants of smoking cessation among these people and to find out how they can be stimulated to quit smoking. A search of the literature revealed no such studies.

\section{Methods}

\section{Recruitment}

Patients recruited were hospitalized in the cardiology wards of four Dutch hospitals during the period from November 1995 until November 1996 and had smoked at least one cigarette per week prior to their admission. Inpatients with unstable complaints (i.e., unstable hemodynamic situation manifested in tightness of the chest and chest pain) and terminal patients were excluded.

Patients were informed about the study by means of a letter and were asked to give their informed consent. Afterward, patients who were willing to participate were asked to complete a questionnaire. It had been agreed with the medical ethical committees of the hospitals that the researcher was not allowed to ask the nonparticipants for their reasons to refuse or about their demographic characteristics.

\section{Questionnaire}

The questionnaire was derived from a Dutch questionnaire used to assess the stages of change and the psycho-social determinants of the general Dutch population (Dijkstra et al., 1996) and from a study of literature on the psycho-social determinants of cardiac inpatients (Breteler et al., 1988; Devalle \& Norman, 1992; Kristeller et al., 1992; Marshall, 1990; McCauley, 1995). 
Attitude was assessed by means of a subscale of 11 advantages (Cronbach's ou = .85) of non-smoking (e.g. "It I stop smaking, my risk of lung cancer will decline", "If I stop smoking, my physical condition will improve"), scored on 4-point scales ranging from no $(0)$ to wery much (3), and a subscale of 4 disadvantages (Cronbach"s $\alpha=57$ ) of norrsmoking (e.g. "If I stop smoking, I will gain weight"). The disadvantages were scored on 4-point scales ranging from no (0) to very much $(-3)$. See Table 2.2 for an overview. Such advantages and disadvantages are referred to as positive and negative outcome expectations in Bandura's Social Learning Theory (Bandura, 1986).

social infuences from partners, children, relatives, cardiologists, friends, colleagues, and ward nurses were assessed by measuring their perceived smoking behavior (Cronbach's $\alpha=50$, see also Table 2), as well as their social support. Smoking behavior of the social environment was scored on a 3-point scale ranging from - 1 (everyone smokes) to 1 (no one smokes). Social support was measured on 7 -point scales and expressed as "Do you experience support or discouragement from your .... towards non-smoking?" (Cronbach's $\alpha=84$ ).

Self-efficacy expectations were measured by elght items on 7-point scales, referring to how difficult smokers thought it would be to refrain from smoking in socially, emotionally, and habitually addictive situations (Kok el al., 1991; Mudde et al., 1995b). These items were expressed as "How easy or difficult do you find it to refrain from smoking in...." (Cronbach's $\alpha=93$ ). (See Table 2.2. for an overview).

Motivational phases in behavior were assessed by the Prochaska and Diclemente typology (Prochaska \& DiClemente, 1992; Prochaska et al., 1992). Questions determining the phases were expressed as "Are you planning to quit within...." and "Did you smoke during your hospital admission?" Three alterations to this typology were necessary to make it fit the situation of cardiac inpatients. First, our sample did not include maintainers because the study only included patients who had smoked in the week prior to their admission. Second, as described in the Introduction, the group of actors was divided into internally and externally motivated actors. Third, due to the small sample size of smokers in the contemplation phase $(N=11)$, they were combined as a group with those in the preparation phase. This resulted in the following four groups: (1) Smokers in precontemplation phase; those who had been smoking since hospital admission and were not planning to quit within the next 6 months.

(2) Smokers in contemplation phase (combined with smokers in preparation phase); those who had been smoking since hospital admission and were planning to quit within the next 6 months.

(3) Extemally motivated actors, those who had not been smoking since hospital admission due to hospita! regulations or for medical reasons and intended to start smoking again as soon als possible.

(4) Intemally motivated actors; those who had not been smaking since hospital admission and were planning to maintain nonsmoking. 
The degree of addiction was measured by the Fagerstrom Test for Nicotine Depen dence (Heatherton, Kozlowski, Frecker, \& Fagerström, 1991), which assesses smoking habits on the basis of questions such as: How much do you smoke? How soon after awakening? Do you find it hard not to smoke in public places were smoking is not allowed? Do you smoke when you are ill? Do you smoke in the morning? Which cigarette is the most difficult to give up? The minimum score was 0 and the maximum 10. Cronbach's $\alpha$ was. 73, which is a satisfactory reliability in comparison with the findings of Heatherton et al. (1991) and Pomerleau et al. (1994).

Demographic measures included age, gender and educational level. Educational level was categorized as (1) primary or basic vocational school, (2) secondary school, (3) higher vocational school, (4) uniwersity.

Finally, some specific characteristics of this population, such as previous admission for cardiovascular disease and diagnosis, were recorded.

\section{Results}

\section{Subjects}

A total of 532 patients signed the informed consent form and completed the questionnaire, while 20 patients $(4 \%)$ refused. Half of the latter came from one hospital ward and had been admitted for only one day, for a specific treatment.

\section{Demographics and Smoking Status}

The 532 participants in the four motivational phases did not differ with regard to age, daily consumption of cigarettes, age at which they had started smoking, educational level or gender distribution. They had the following characteristics.

Precontemplation. The average age of the 34 participants in this phase was 56 years. They smoked an average of 19.9 cigarettes a day and had started at an average age of 15.6 years.

Contemplation. The average age of the 56 participants in this phase was 55 years. They smoked an average of 15.6 cigarettes a day and had started at am average age of 17.4 years. Externally motivated actor phase. The average age of the 147 participants in this phase was 56 years. They used to smoke an awerage of 19.1 cigarettes a day and had started at an average age of 17.6 years.

Internally motivated actor phase. The average age of the 274 participants in this phase was 55 years. They used to smoke 20 cigarettes a day and had started at an average age of 17.3 years. 


\section{Differences between the Groups}

Table 2.1 summarizes the differences between the four groups representing the various motivational phases, analyzed by means of variance analysis on standardized scores with Tukey-HSD contrasts (Hays, 1981). With regard to hypothesis 1, externally motivated actors perceived significantily fewer positive consequences of non-smoking, showed lower levels. of self-efficacy expectations and experienced less social support than internally motivated actors. However, they did not perceive significantly more disadvantages of nonsmoking.

With respect to hypothesis 2, internally motivated actors expected significantly more advantages of nonsmoking than smokers in precontemplation. Smokers in contemplation scored higher on the pros than smokers in precontemplation, although the difference was not significant. The internally motivated actor group expected fewer cons than smokers in precontemplation. Social support was positive only for internally motivated actors. Smokers in contemplation reported neither support nor discouragement from their environment.

With regard to hypothesis 3 , smokers in precontemplation had the lowest levels of social support and differed significantly from internally motivated actors. Smokers in contemplation did not differ significantly from the latter group, but they did differ from smokers in precontemplation in the level of perceived support toward nonsmoking. Only the externally motivated actors reported significantly less smoking behavior in their environment. With respect to self-efficacy expectations, internally motivated actors experienced significantly higher levels of self-efficacy than the other groups (hypothesis 4).

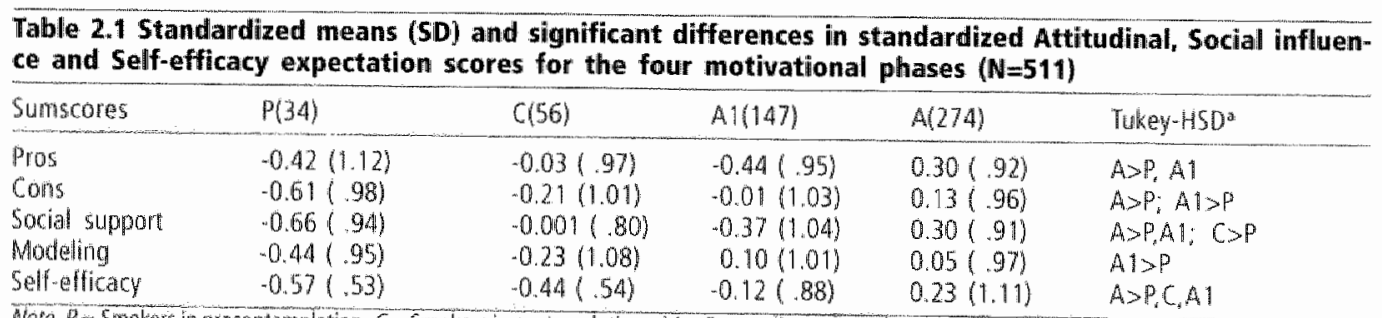

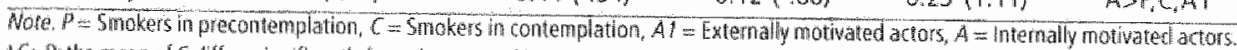

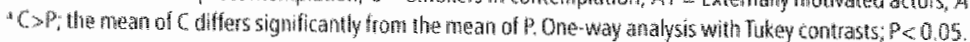

\section{Attitudinal Differences}

Differences in group means with respect to beliefs about the advantages and disadvantages of nonsmoking were analyzed with univariate analysis of variance. The Tukey-HSD method was used for the pairwise comparisons if the overall $F$ test was found to be significant at $p<.05$ (Hays, 1981). The same procedure was used for the scores on social support, perceived behavior of important others, self-efficacy expecta- 
tions, and intentions. On the whole (see Table 2.2), internaly motivated actors were significantly more convinced of the positive consequences (pros) of nonsmoking than the other groups (hypothesis 2). The difference was particularly obvious in comparison with externally motivated actors and smokers in precontemplation. In general smokers in contemplation did not differ from internaly motivated actors with respect to their perception of the pros of nonsmoking. However, smokers in contemplation were significantly less convinced than internally motivated actors that quitting could reduce their heart complaints, that quitting could reduce the fear of recurrence of the heart complaints, and that it could reduce the risk of hospital readmission.

With respect to the negative consequences (cons) of nonsmoking (Table 2.2), the internally motivated actors were significantly less convinced than precontemplators that nonsmoking would make it harder for them to relax. Both actor groups were less convinced that quitting would result in withdrawal symptoms. There were no differences between the groups in their perception of gaining weight and missing the conviviality of smoking.

\section{Differences in Social Influences}

In general, all inpatients experienced weak levels of social support (see Table 2,2). With respect to support from their partners, only the internally motivated actors reported a moderate level of support. Furthermore, all groups scored higher on social support by the medical staff (cardiologist and ward nurses) than on support by others. With regard to all sources of social support, internally motivated actors experienced significantly more support than smokers in precontemplation (hypothesis 3). Smokers in contemplation also experienced a moderate level of social support.

Overall, the role model of others was judged to be neutral (scores all close to zero). There were no significant difierences between the groups in their perceptions of the smoking behavior of partners, children, relatives, cardiologists, friends, colleagues, or ward nurses.

\section{Self-Efficacy Differences}

Table 2.2 shows, that smokers in precontemplation, smokers in contemplation, and externally motivated actors generally perceived nonsmoking behavior as more difficult than did internally motivated actors (hypothesis 4). Scores on situational selfefficacy expectations showed nearly the same picture (see Table 2.2). In comparison with the other groups, internally motivated actors expected significantly fewer difficulties in refraining from smoking at parties where others are smoking, in situations of tension or 


\section{Table 2.2 Means and significant differences in Attitudinal, social influence,} Self-efficacy and Intention scores for the four motivational phases.

\begin{tabular}{|c|c|c|c|c|c|}
\hline Attitude flems & $P(28)$ & $C(54)$ & A $1(129)$ & $A(237)$ & Tukey-HSD* \\
\hline \multicolumn{6}{|l|}{ Pros of not smoking } \\
\hline My heart complaints wall be reduced & 1.14 & 1.48 & 1.54 & 2.11 & $A>P_{n} C_{n} A 1$ \\
\hline The risk of lung cancer will be reduced & 1.85 & $2: 17$ & 1.76 & 2.23 & $A>A 1: C>D$ \\
\hline Less coughing & 1.45 & 1.53 & 1.37 & 1.76 & $A \rightarrow A_{1}$ \\
\hline More satistied about myself & 4.63 & 2.02 & 1.77 & 2.22 & $A>A 1, P$ \\
\hline Less fear of recurrence of heart complaints & 1.03 & 1.46 & 1.36 & 1.93 & $A>P, C, A 1$ \\
\hline Seeing myself as a sensible person & 2.15 & 2.29 & 2.03 & 2.35 & $A>A 1$ \\
\hline Risk of rehospitalization will be reduced & 1.50 & 1.56 & 1.55 & 2.00 & $A>P, C, A 1$ \\
\hline Less inconvenience for the people around me & 2.01 & 204 & 1.73 & 2.03 & $A>A !$ \\
\hline Better physical condition & $\$ .75$ & 1.96 & 1.73 & 1.99 & $A>A 1$ \\
\hline Better physical health for norwsmokers in my environment & 1.55 & 1.76 & 1.16 & $\$ .69$ & $A>A 1 ; C>A=$ \\
\hline $\begin{array}{l}\text { I am astianed thiat I have to smoke } \\
\text { Cons of not smoking }\end{array}$ & 0.80 & 0.87 & 0.53 & 0.79 & \\
\hline It will be harder to rela $x$ & -1.65 & -1.49 & -1.35 & $-1,12$ & $A>P$ \\
\hline I will gain weight & -1.39 & -1.31 & -1.22 & -1.32 & \\
\hline I will miss the conviviality of smoking & -1.88 & -1.59 & -1.56 & -1.43 & \\
\hline I whll experience withdrawal symptoms & -1.86 & -1.44 & -1.15 & -1.04 & $A>P: A 1>P$ \\
\hline Social influence items & $P(32)$ & $(156)$ & $A 1(146)$ & $A(264)$ & \\
\hline \multirow{2}{*}{\multicolumn{6}{|c|}{$\begin{array}{l}\text { Support or discouragement towards non smoking } \\
\text { Partner }\end{array}$}} \\
\hline & 0.35 & 0.96 & 0.85 & 1.72 & $A>P, C, A$ \\
\hline Children & 0.70 & 1.33 & 0.92 & 1.60 & $A \geq P, A 1$ \\
\hline Relatives & 0.61 & 1.14 & 0.78 & 1.42 & $A>P, A l$ \\
\hline Cardiologist & 0.94 & 1.92 & 1.17 & 1.94 & $A>P, A 1: C>P_{n} A 1$ \\
\hline Friends & 0.38 & 1.18 & 0.73 & 1.35 & $A>P, A \mid C>P$ \\
\hline Colleagues & 0.19 & 0.65 & 0.45 & 0.73 & $A>P: A l$ \\
\hline Ward nurses & 0.73 & 1.46 & 1.12 & 1.87 & $A>P_{*} A^{1}$ \\
\hline \multicolumn{6}{|l|}{$\begin{array}{l}\text { Perceived smoking behavior of: } \\
\text { Partner }\end{array}$} \\
\hline Parther & -0.33 & -0.13 & 0.07 & -0.04 & \\
\hline Children & 0.12 & 0.07 & 0.22 & 0.17 & \\
\hline felatives & 0.03 & -0.05 & 0.25 & 0.18 & \\
\hline Cardiologist & 0.09 & 0.15 & 0.05 & 0.10 & \\
\hline thiends & -0.23 & 0.20 & 0.07 & 0.04 & \\
\hline Colleagues & 0.19 & -0.01 & -0.02 & 0.12 & \\
\hline Ward nuirses & 0.07 & 0.11 & 0.07 & 0.10 & \\
\hline thens on self-eflicacy expectations & $P(34)$ & $C(56)$ & $A 1(147)$ & $A(274)$ & \\
\hline $\begin{array}{l}\text { How difficult ar easy it would be to refrain from smoking } \\
\text { Is it difficul or easy not to smoke when.. }\end{array}$ & -2.59 & -2.33 & -1.98 & -1.57 & $A>P, C, A$ \\
\hline \multirow{7}{*}{ 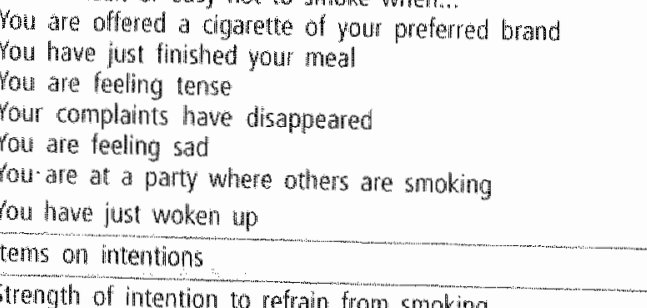 } & -2.12 & -2.13 & -1.71 & .1 .15 & $A>P B C A$ \\
\hline & -2.51 & 2.24 & -1.86 & -1.39 & $A>B, A$ \\
\hline & -2.36 & -2.59 & -2.14 & -1.72 & $A>C, A$ \\
\hline & -2.23 & -1.83 & -1.29 & 0.82 & $A>P, C, A I, A \backslash P$ \\
\hline & -2.35 & -2.17 & -1.73 & -1.32 & $A \times P, C, A 1$ \\
\hline & -2.22 & -2.12 & -1.98 & -1.32 & $A>B, C, A$ \\
\hline & .232 & -1.69 & -1.27 & -0.87 & $A>P ; C ; A 1>P$ \\
\hline terns on intentions & $P(33)$ & $C(54)$ & A. 143$)$ & $A(26 \pi)$ & \\
\hline \multirow{2}{*}{$\begin{array}{l}\text { trength of intention to refian from smoking } \\
\text { fres discharge from hosplat } \\
\text { iobability of reframing from snoking } \\
\text { fler discharge from hosphtal }\end{array}$} & 3.75 & 6.27 & 6.03 & 8.64 & $\begin{array}{l}A P P: C>P \\
A>P, C, A 1\end{array}$ \\
\hline & 3.19 & 6.43 & 5.89 & 8.12 & $\begin{array}{l}A>P, C, A 1: \\
A 1>P, C>P\end{array}$ \\
\hline
\end{tabular}

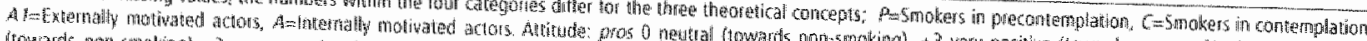

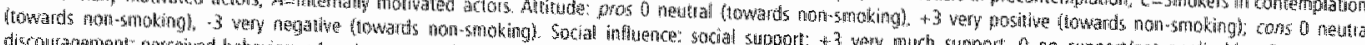

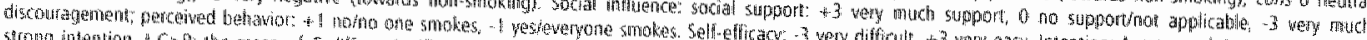

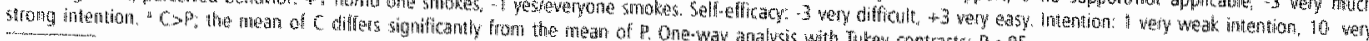


sadness, after finishing a meal, or upon being offered a cigarette of their own preferted brand. In some situations, for example after their complaints had disappeared, externally motivated actors expected far fewer difficulties with nonsmoking than did smokers in precontemplation. In general, patients expected the greatest urge to smoke in tense situations. Overall, all groups reported low levels of self-efficacy.

\section{Differences in Intentions}

Smokers in contemplation and both groups of actors had significantly stronger intentions than smokers in precontemplation about not smoking after hospital discharge (see Table 2.2). The same pattern was found with respect to the probability of refraining from smoking. Furthermore ${ }_{n}$ internally motivated actors reported significantly stronger intentions to maintain nonsmoking behavior than all other groups. They also reported a greater probability of remaining nonsmokers after hospital discharge.

\section{Discussion}

This study assessed whether externally and internally motivated cardiac inpatients in action differed from each other with respect to their psycho-social determinants. Furthermore, it analyzed differences in psycho-social factors between subjects in the warious motivational phases measured according to the Transtheoretical Model (Prochaska \& DiClemente, 1992; Prochaska et al., 1992). Finally, the study analyzed whether the $O$ pattern that had been found in earlier research could be replicated for Dutch cardiac inpatients.

The findings support the first hypothesis, i.e, that the group of actors consists of two subgroups which differ in their psycho-social determinants and their intentions to refrain from smoking after hospital discharge. Internally motivated actors experienced more positive consequences of nonsmoking than externally motivated actors did. Furthermore, the latter experienced less social support toward maintaining the nonsmoking behavior and experienced significantly lower levels of self-efficacy than the internally motivated group. These results could be explained by the fact that it was external circumstances which forced these patients into their nonsmoking behavior, rather than their own matiwation. These findings suggest that externally motivated actors have to be approached in a different way than internaly motivated actors. Unilke what was suggested in the paper by Kristeller et al. (1992), externally motivated actors cannot be seen as smokers in precontemplation, because they reported fewer cons of nonsmoking and significantly higher levels of intention toward non-smoking.

With regard to the second research question, it was expected that subjects in the different motivational phases would differ in their determinants of behavional inten- 
tions (hypothesis $2,3,4$. Athough several significant differences were found the results were not all in line with the findings of previous research. In contrast with hypothesis 2 and other findings (De Vries \& Backbier, 1994; DiClemente et al., 1991; Dijkstra et al. 1996; Strecher, DeVellis, Becker, \& Rosenstock, 1986; Velicer, DiClemente, Prochaska, \& Bandenburg, 1985f, smokers in precontemplation did not show a significantly lower perception of the pros of nonsmoking than did smokers in contemplation. No previous studies were found to support this finding and the only explanation seems to be the small sample sizes of the two groups, since the means did differ in the present study. In line with studies by De Wries \& Backbier (1994) and Dijkstra ef al. (1996), no differences were found between the attitudes of smokers in contemplation and those of internally motivated actors. However, there were interesting differences between these two groups with respect to their beliefs about the reduction of heart complaints, the fear of recurrence of complaints, and the risk of hospital readmission. These findings suggest that smokers in contemplation are less convinced of the relationship between their complaints and their smoking behavior than internally motivated actors.

With respect to social influence thypothesis 3), internally motivated actors experienced significantly more social support than smokers in precontemplation. In addition, smokers in contemplation experienced more support than smokers in precontemplation. No significant differences were found with respect to madeling.

With regard to self-efficacy expectations, intenally motivated actors perceived significantly fewer difficulties with nonsmoking than all other groups (hypothesis 4). in contrast with the fincings by Snow et al. (1992) and DiClemente et al. (1991), smokers in contemplation did not report higher self-efficacy expectations than smokers in precontemplation. This could be due to small sample sizes. However, our results do confirm hypothesis 4 and the results of previous Dutch research (De Vries \& Backbier, 1994; Dijkstra et al. 1996). The findings of this study suggest a Q-shaped pattern with respect to smokers in precontemplation, smokers in contemplation and internally motivated actors (hypothesis 5, Figure 2.3).

The results of the present study generally support strategies previously suggested by other researchers concening behavioral change programs (Brug et al «, 1997; De Vries \& Backbier, 1994; De Vries \& Mudde, 1998; De Vuies et al., 1998; DiClemente et al., 1991; Dijkstra et al., 1996; Velicer et al., 1985). Smokers in precontemplation need attitude enhancing information (e.g. information on positive consequences for personal health) to become more convinced of the advantages of nonsmoking. This would help them move to the contemplation phase. Smokers in contemplation need selfefficacy emhancing information to become convinced of their ability to quit (e.g., intormation showing them how to quit and how to cope with difficult situations). However, unlike what has been suggested earlier, cardiac inpatients in the contemplation phase will also need attitude enhancing information on the positive consequences of smow king cessation for their prognosis, since the results of the present study suggest that smokers in contemplation were not convinced of the relationship between the com- 
plaints and their smoking behavior. This proposition is supported by recent findings of Difkstra and colleagues (1998b). These researchers used an experimental design to show that people in the contemplation phase, in moving toward the action phase benefit most from an intervention containing attitudinal and self-efficacy enhancing information. Moreover, longitudinal research by Dijkstra et al. (1998a) suggested that such an intervention might be most effective on smoking cessation. To enhance beha. vioral change in externally motivated actors, patients have to become internally motivated and have to acquire more positive self-efficacy expectations. This might be achieved by convincing these patients of the benefits of quitting (attitude enthancing information, e.g., quitting smoking reduces your risk of recurrence of heart complaints by $50 \%$ ) combined with self-efficacy enhancing information (e.g., how to cope when you feel tense). Modeling with guided enactment would be the optimum method for enhancing self-efficacy (Bandura, 1986).

The present study has some limitations. First, the study yielded only cross-sectional data, making it difficult to assess whether differences in determinants preceded or followed the motivational phases. A Dutch (De Vries \& Mudde, 1998) and an American (Prochaska, DiClemente, Velicer, Ginpil, \& Norcross, 1985) study have provided langitudinall support for the $\theta$ pattern in the general population "but since the present study involved a specific population, longitudinal research will still be necessary. A related problem is the validity of the measurement of motivational phases according to the $T M$, since the empirical evidence is mainly based on cross-sectional research, which makes subdivisions and cut-off points rather arbitrary. This is particularly important for the present study because the literature reveals only very little information on the mativational phases of people suffering from cardiac diseases. Two studies (Kristeller et al., 1992; Ockene et al., 1992) have provided suggestions for the measurement of motivational phases of change among cardiac patients, based on the typology of Prochaska and DiClemente. However, their suggestions were not followed, because they classified motivational phases by combining self-reported smoking behavion and attitudes toward quitting. We believed that motivational phases had to be measured by self-reported smoking behavior combined with plans to quit (e.g., "Are you planning to quit within..." $)$. Longitudinal research among cardiac patients is recommended to make subdivisions and cut-off points more valid. In addition, several other studies have indicated that the measurement of phases using the TM algorithm needs revision (Lechner, 1998; Lechner, Brug, De Vries, Van Assema, \& Mudde, 1998). Second, the small number of smokers in the precontemplation and contemplation phases may have reduced our ability to detect differences between the groups (type II error). Moreover, the group of smokers in contemplation mainly consisted of smokers in preparation. It is therefore recommended for future research to include sufficient numbers of cardiac inpatients in all motivational phases. Third, the reliability of the modeling subscale was modest (Cronbach's $\alpha=50$ ). The results showed only that externally motivated actors perceived a significantly better example in their environment than smokers in precon- 
templation. Because of the modest reliability, these results should be interpreted with caution. A future study will have to pay attention to the reliability of the modeling scale. Fourth, no maintainers or selapsers, were included in the present study. Effective aftercare and a relapse prevention strategy therefore require further research for these groups. Fifth, the present study design did not allow comparison between participants and patients who refused to participate, and could thus not assess the possibility of nonresponder bias. Finally, the measurement took place within 96 hours after admission. It is conceivable that patients become less motivated to quit smoking as they start to feel better. For the purpose of intervention development, it would be interesting to know how stable the patients' considerations are over the period from admissian to discharge.

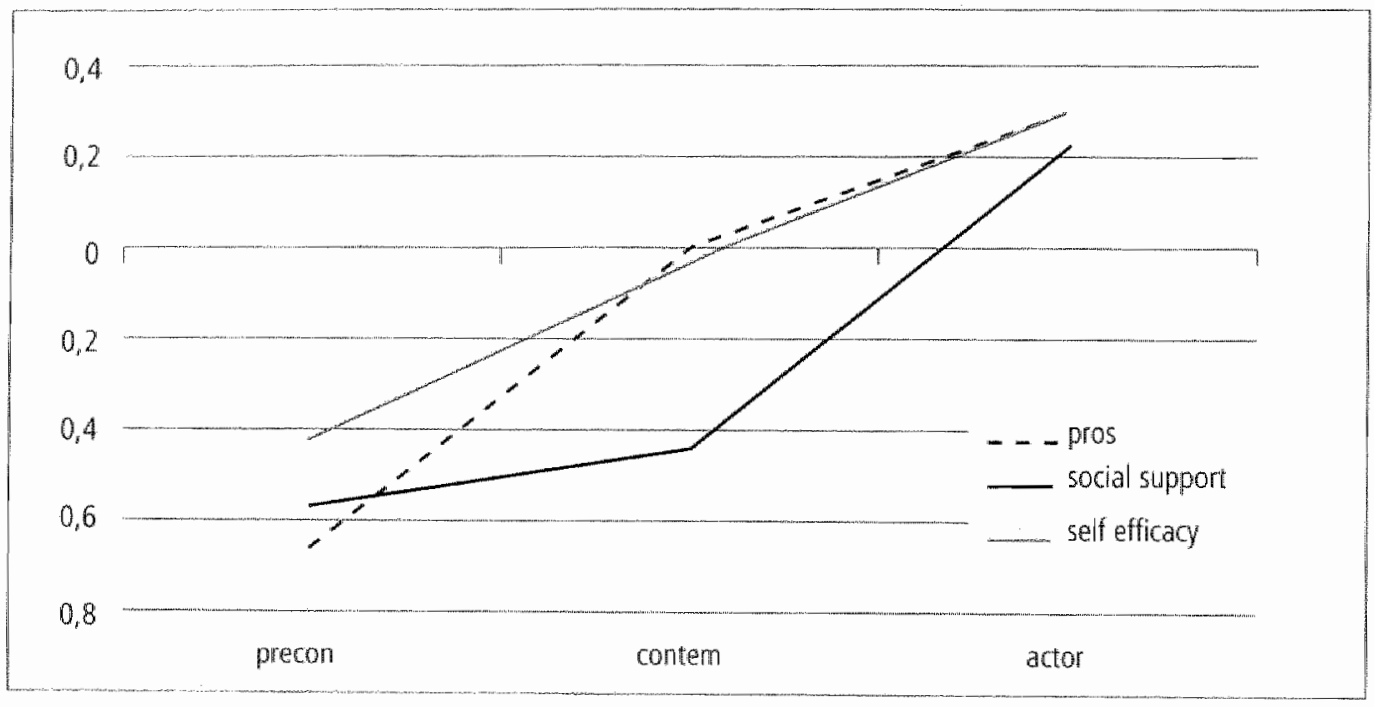

Figure 23.

The e pattem for the smokers in precontemplation, contemplation and for internally motivated ators.

\section{Conclusions}

With respect to the development of interventions for the population of cardiac inpatients, the following suggestions cam be derived from the present study.

First, the differences in psycho-social determinants between internally and externally motivated actors imply that the latter subgroup has to be acknowledged as a separate group in developing and implementing interventions. 
Second, attitude enhancing information should be given to smokers in precontemplation to motivate them to contemplate behavioral change. Self-efficacy enhancing information should be made avalable to subjects in the contemplation phase to mot:vate them to start changing their be havior. In addition, the latter also need information on the relationship between theil complaints and the smoking behavior linformation on pros of quitting).

Finally, the psycho-social differences between the various motivational phases lead to the recommendation to develop phase-tailored interventions. At present, it is unclear whether such an intervention should be implemented by interpersonal counse. ling, or whether written or computer-generated materials tailored to the motivational phase would also have a satisfactory effect, while requiring less manpower. 


\section{EVALUATION OF A NURSE-MANAGED MINIMAL- CONTACT SMOKING CESSATION INTERVENTION FOR CARDIAC INPATIENTS*}

\section{Abstract}

Background. This study examined the effectiveness of a nurse-managed milimalcontact smoking cessation intervention for patients hospitalized for cardiac disease.

Methods. A Pretest-Posttest quasi-experimental design was used. Patients who smoked prior to admission to cardiac wards of 5 hospitals $(N=388)$ received the intervention, whereas smoking patients in 6 other hospitals were given usual care $(N=401)$. The intervention was initiated at the hospital and continued after discharge. The core elements were stop-smoking advice from the cardiologist; a short bedside consultation with a nurse; administration of self-help materials and aftercare by the cardiolo gist. Smoking cessation was assessed after 3 months by self-report.

Results. Lagistic regression analysis excluding dropouts, controlling for covariates including baseline differences showed significant intervention effects (one-tailed significance test) on point prevalence abstinence (OR $=2.11$ ) and continuous abstinence $(O R=1.41)$. Intention-to-treat analysis including dropouts as smokers showed a significant effect on point prevalence abstinence (OR=1.35).

Conclusions. Compared to usual care, the low-intensity smoking cessation intervention for cardiac inpatients was more effective in achieving smoking cessation. However, the small effects and the process evaluation suggest that improvements are needed.

*In press as: Bolman, C., De Vries, H. Van Breukelen, G. Evaluation of a nurse-managed minimal-contact smoking cessation intervention lor cardiac inpatients. Health Education Research. 


\section{Introduction}

Smoking cessation is known to improve the prognosis of patients with coronary heart disease (CHO) more than any other treatment (Deckers et al., 1994; Mehta \& Eagle, 1998; Smith, 1997; U.S. DHHS, 1990): A meta-analysis has shown that smoking cessation among patients with newly established $\mathrm{CHD}$ reduces the risk of mortality after one year by almost 50\% (Deckers et al.. 1994), while smoking cessation after a first myocardial infarction reduces the risk of recurrence by half (Mulcahy. 1983; Voors et al., 1996).

Despite these promising effects, 20-60\% of cardiac patients who smoked prior to their haspital admission persist in their smoking behavior or relapse after hospital discharge (Deckers et al., 1994; Huijbrechts et al., 1996; Ockene et al., 1992; Orleans et al., 1990; Perkins, 1988; Rigotti et al., 1994; Rigotti et al., 1991; Rose \& Colwell, 1992; Taylor et al., 1990).

Admission to a cardiology ward is often referred to as an excellent opportunity for a smoking cessation intervention, because at that time patients are more aware of their personal vulnerability to the dangers of smoking, are highly motivated to quit and are more receptive to a smoking cessation intervention than in other circumstances (Emmons \& Goldstein, 1992; Miller, Smith, DeBusk, Sobel, \& Taylor, 1997; Orleans et al., 1993b; Orleans \& Ockene, 1993; U.5. DHHS, 1996). In addition, patients often have been smoke-free for a period of days and may therefore be more willing to continue nonsmoking.

Unfortunately, coronary care nurses and cardiologists show little effort in supporting patients to quit smoking. Although cardialogists usually adwise patients to stop smoking (Van Berkel et al., 1999a), only a few actually support patients in the smoking cessation process (Duncan et al., 1991; Emmons \& Goldstein, 1992; Frank, Winkleby, Aitman, Rockhill, \& Fortmann, 1991; Goldstein et al., 1987; Sanders et al., 1986; Tessier et al., 1995). Dutch research has revealed that only one-third of all coronary care nurses addressed the smoking belhavior of their patients, while only $10 \%$ of the nurses and specialists assisted patients in their attempts to quit (N:PO, 1995; Wiebing \& Baan, 1997).

The objective of the present study was to assess the effectiveness of a nurse-managed minimal-contact smoking cessation intervention for patients hospitalized for CHO. A minimal-contact approach was chosen for three reasons. First, a needs assessment among Dutch coronary care nurses has revealed that nurses were only willing to spend 15 to 30 minutes on smoking cessation assistance (unpublished data). Second, cardiac inpatients have expressed minimal interest in formal treatment (Emmons \& Goldstein, 1992; Fiore et al., 1990; Glynn, Boyd, \& Gruman, 1990), while showing considerable interest in self-help guidance and brief counseling (Emmons \& Goldstein, 1992). Third, several reviews have suggested that brief treatments including stopsmoking advice and brief counseling, combined with self-help and brief follow-up care are acceptable to patients, cost-effective and feasible within existing hospital practices (Miller, 1993; Orleans, Glynn, Manley, \& Slade, 1993a; Orleans et al., 1993b). Meta- 
analyses constituting the basis for the development of the Clinical Pactice Smoking Cessation Guidelines, released in the U.S. in 1996 (U.5. DH.HS, 1996) and in the U.K. in 1998 (Raw, Mckeill, \& West, 1998) confirmed that these steps were essential and ef. fective.

The Minimal Intervention Strategy for cardiac inpatients (C-MIS), which is the topic of the present paper, is based on the Minimal Intervention Strategy for smoking cessation in general practice (MIS). MS has proved its efficacy and feasibility in general practice in a large randomized controlled trail, where it resulted in twelve-months cessation rates of $17.5 \%$, compared to $8.5 \%$ in the usual care group (Pleterse et al. 1994). MI $\varsigma_{4}$ which consists of 5 to 10 minutes of stage-tallored personal counseling combined with self thelp quidance, is based on the Health Counseling model, a model developed as a specific tool melping health care providers to prepare patients for and assist them in the process of behavioral change (Gerards, 1993; Gerards, 1997; Gerards \& Hospers, 1991).

Although several studies have reported on the efficacy of smoking cessation programs for cardiac inpatients (Johnson et al. 1999; Ockene et al., 1992; Rigotti et al.; 1994; Rosal et al. 1998; Taylor et al., 1990) and for hospitalized patients in general (Miller et al., 1997; Orleans et al 2,1990 ; Rigotti et al., 1997; Sirota et al., 1985; Strecher et al., 1985; Taylor et al., 1996), this mainly concerned interventions involving at least one hour in-hospital counseling, combined with intensive follow-up contacts by telephone after discharge. The intervention in the current study "however, involved a minimal approach, tested under typical practice conditions.

Besides the effectiveness study, a process evaluation was conducted to assess the fidelity of the delivery of the intervention by measuring the level of utilization of the intervention and the quality of its implementation. Process evaluations facilitate understanding why interventions were successful or failed. Moreover, their results can be viewed as accumulating evidence together with the results of the effectiveness evaluation. This combination of evidence indicators is referred to as data-triangulation, which is especially important in quasi-experimental effectiveness studies (Green \& Kreuter, 1991; MacDonald \& Muir, 1996; Nutbeam, 1998; Nutbeam, 1999; Tones, 1997; Windsor et al, 1994).

\section{Methods}

\section{Design, Study Population and Procedure}

Twenty-one hospitals were approached for participation in the study by contacting the managers of the cardiac wards. Ten hospitals refused to participate for various reasons, induding a merger of wards, current participation in other time-consuming studies, staff turnover and personnel shortage. Initially, the remaining eleven hospitals 
were selected for random assignment to the intervention or control group. However two hospitals only wanted to serve as controls. Similarly, two other hospitals were willing to co-operate only if they were induded in the intervention group:. This situation resulted in self-selected assignment of four hospitals and random assignment of the remaining seven hospitals, which meant a Pretest-Posttest quasi-experimental design ex post (Rossi \& Freeman, 1993). Five hospitals constituted the intervention group, in which patients were assisted by means of the C-MIS. Six other hospitals served as the control group, in which patients received usual care. Patients nested in hospitals were the unit of analysis.

From November 1995 to May 1997, smoking patients admitted for CHD to the cardiac wards of the selected hospitals were recruted by ward nurses. Patients who reported having smoked at least one cigarette in the seven days prior to admission, and were not in a life-threatening condition or in a terminal phase were eligible for inclusion. During the enrollment period, patients" smoking behavior in the seven days prior to adrnission were routinely assessed by murses at the intake. For emergency admission, the patient's smoking behavion was assessed later on, but within 96 hours from admission. The two other criteria were also judged by nurses. Every day during the briefing at shift change it was communicated whether a patient was eligible for study inclusion or whether the smoking behavior of a patient still needed to be assessed. internal program co-ordinators coordinated and supervised the process of inclusion. These co-ordinators and nurses were explicitly instructed to ask all patients who were eligible for inclusion, and to register refusals. Patients were informed about the study by mears of a letter provided by ward nurses and were asked to volunteer for the study, including the possibility of being a control group member. Patients who were willing to participate had to give informed consent.

Baseline measures were obtained by means of a questionnaire administered by ward nurses within a few hours of the patients signing the informed consent. Outcome measures were assessed by means of questionnaires malled to patients' home addresses three months after inciusion. Postal reminders were sent thee and eight weeks after the mailing of the questionnaire. If no response occurred, patients were phoned up by the researcher with the request to return the questionnaire.

\section{Intervention}

The MIS served as a blueprint for the intervention used in the present study. A few aiterations were however made for the target population. The first was the inclusion of a stop-smoking advice by the cardiologist, which has been found to be essential for Cardiac inpatients (Burling et al., 1984: Fiore, 1997; Fowler, 1997; Miller, 1993; Orleans \& Ockene, 1993; Onleans et al., 1990; Raw et al. 1999). The second alteration concerned the aftercare, which is not structurally embedded in MIS. In the C.MIS, it was embedded in the patient's check-up visit at the cardiology outpatient clinic 4 to 6 
weeks after discharge since a large proportion of cardiac inpatients are known to rew lapse after discharge (Breteler et al., 1988; Kottke et al., 1988; Oneans et al., 1990; Rigotil et al., 1991; Taylor et al. "1990).

The final protocol consisted of the following (see Figure 3.1). Firstly, patients received stop-smaking advice from their cardiologists. Secondly, a ward nurse assessed the patients" smoking behavior, theil degree of addiction and their motivation to quit. This "readiness for change" formed the base of further counseling provided by nurses. Step

\section{Nurse}

\section{Cardiologist}

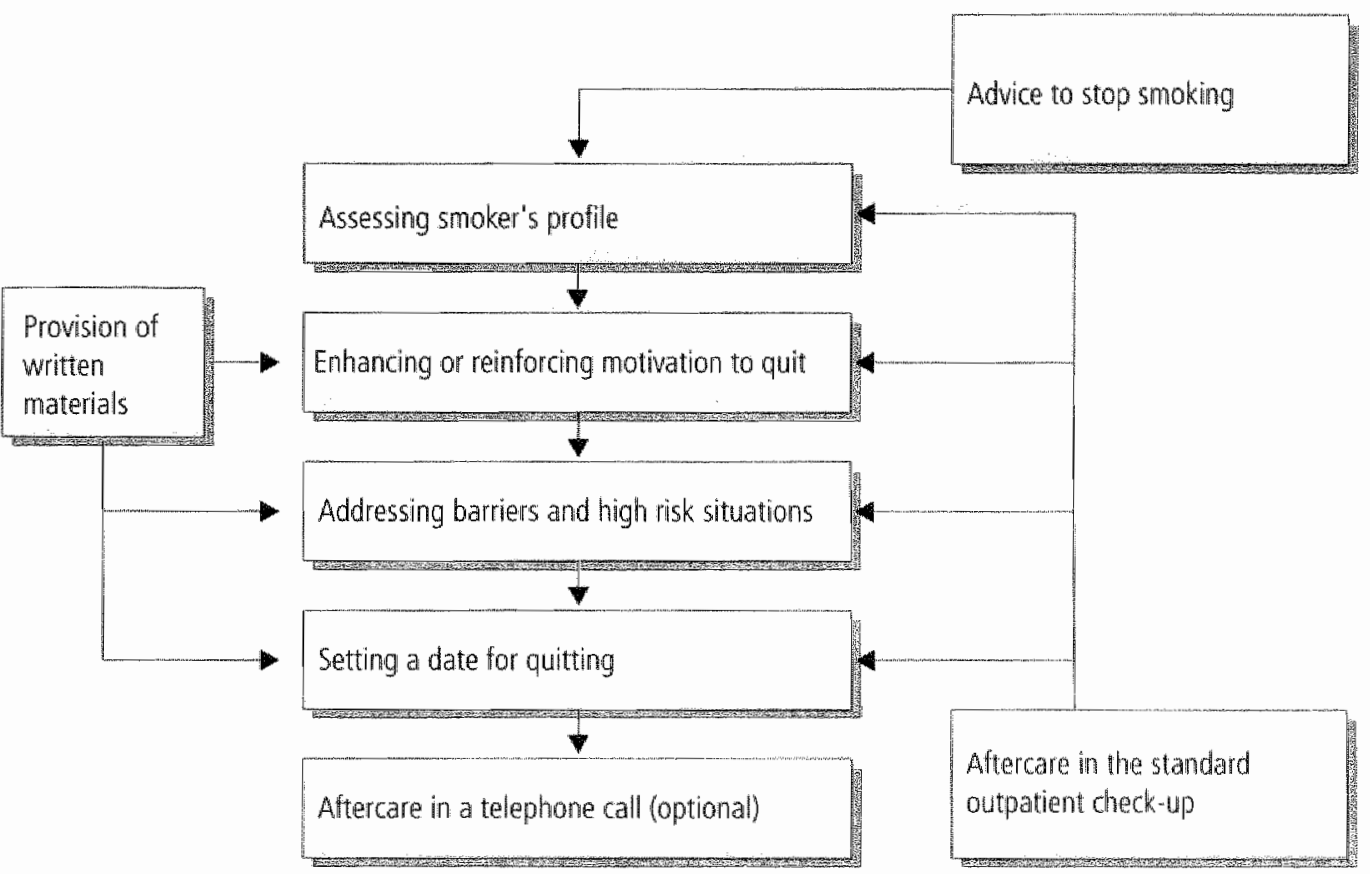

Figure 3.1. C-MIS protocol.

3 was only employed if a patient was unmotivated to quit: the nurse focused on the positive and negative consequences of smoking cessation by means of strategies deri. yed from motivational interviewing (Miller \& Rolnick, 1991). If patients were already motivated to quit, nurses could proceed to step 4 , addressing the perceived barriers (expected problems and high-risk situations) and perceived self-efficacy expectations for smoking cessation. The nurse helped the patient select problem areas (e.g. withdrawal symptoms, stress, mood states). Subsequently, the patient was encouraged to consider what would work to cope and was helped to clevelop coping strategies. If a 
patient was motivated to quit and prepared to overcome high-risk situations, the nurse could proceed to step 5: encouraging the patient to set a date for quitting. Depending on the situation, nurses could complete the steps 2 to 5 in one or more (short) conversations. Before the patient's discharge from hospital, the nurse discussed the patient's progress and experiences so far. After discharge, the cardiologist addressed the patients' smoking behavior again at the cardiology outpatient clinic, to prevent relapse or to motivate a patient to make (another) attempt to quit. To remind the cardiologist, patients' hospital charts were stamped with a prompt and included a short report on the content of the in-hospital part of the intervention (on a so-called intervention card). In addition, a copy of the intervention card and a letter in which the protocol was explained were sent to the patient's general practitioner. in the letter the general practitioner was asked to pay attention to the patient's smoking behavior if visiting the general practice after hospital discharge.

Patients also received a self-help manual and a brochure to read in the hospital and to take home. The self-help manual was partly based on the American Lung Association's "Freedom From 5moking" guide (Strecher, Rimer, \& Monaco, 1989), structured in the same way as the protocol and pretested and evaluated in two Dutch projects (Mudde ett al., 1994; Willemsen \& De Vries, 1995). The brochure focused on the relationship between smoking and the progression of CHD. In the case of more severe nicotine addiction or if a patient preferred intensive treatment, nurses pointed out possibilities to participate in a smoking-cessation group program after hospitalization at a cost of $\$ 45$. Initially, the protocal also included an optional telephone call by nurses two weeks after discharge from hospital. Ultimately, ward managers refused to indude this due to time constraints.

Patients of the control group received usual care, which consisted of occasional attention to their smoking behavior. There was no organized intervention.

\section{Training on C-MIS}

All the nurses in the intevention group received a standardized two-hour training. The training included information on the rationale of and the theory behind the intervention. The protocol was explained and practiced in roleplays. In addition, every ward received a training manual with instructions and a poster with a description of the protocol, while every nurse got a pocket-size reminder chart including an overview of the protocol and standardized messages on the pros of quitting and how to cope with high-risk situations. To ensure quality control, each ward appointed two internal program comordinators who supervised the interventions and to whom nurses could turn with questions. Moreover, meetings between the co-ordinators and the researcher were held every three months to discuss progress, difficulties and experiences. Although cardiologists were not specifically trained for their part of the intervention, they received instructions from the internal program co-ordinators, a letter in which the protocol was explained and a flowchart with a description of the protocol and brief phase-related instructions. 


\section{Measurements}

At follow-up, experimental patients" exposure to the intewention components (process evaluation) was assessed by asking whether the patient had laked with the nurse (during hospitalization) and the cardiologist (at the outpatient dinic) about smoking cessation and whether they had received and read the sel-help materials. The latter was assessed separately for both materials by asking: "To what extent did you read the .......?" (answer scale ranging from "not read it' (1) to 'read it all (10)). Questions referring to the personal counseling included: "Has the nurse spoken with you about smoking cessation during your hospital stay?" (yesino) and "Has the cardiologist assessed your smoking habits at the outpatient dinic visit?" (yes/no). Furthermore, experimental patients were asked whether they had perceived more pros and less cons after the conversation with the nurse, whether they had learned how to cope with difficult situations and how to obtain social support (answer scale ranging from "not at all' (1) to "to a large extent" (10)". Process evaluation questions were derived from program exposure measurements in earlier Dutch smoking intervention studies (Dikkstra et al., 1998b; Mudde, De Vries, \& Dolders, 1995a; Willemsen et al., 1998). To obtain the patients" evaluation of the intervention, patients in the intervention group were asked to rate the in hospital and outpatient dinic parts of the intervention separately on an evaluation scale from poor (1) to excellent (10).

To assess whether patients in the intervention group had experienced greater attention for their smoking behavior than patients in the control group, the latter were also asked whether they had spoken about smoking with the cardiologist and the nurse. Patients in both groups were furthermore asked whether the conversations with these two types of health care providers had motivated them to quit or to sustain their attempt to quit. An example of such a question is." "Has the conversation with the nurse motivated you to make an attempt to quit?" (answer scale ranging from "not at all" (1) to 'to a large extent' (10)).

The primary outcome measurement on intervention effectiveness was self-reported point prevalence abstinence ("Have you smoked during the last seven days, even if only one puff?" (yes/no)). The secondary outcome was self-reported continuous abst:nence (no smoking since hospital discharge).

At baseline, nurses documented the patients' reason for admission according the following categories: myocardial infarction; angina pectoris; decompensatio cordis; dysthythmias; coronary angioplasty; and a remaining category other.

The baseline questionnaire that patients had to complete assessed prior hospital admission for cardiovascular disease (CVD); Fagerström Test for Nicotine Dependence (FTND) (Heatherton et al., 1991); sociodemographics (age, gender \& educational level: motivational phases of behavioral change and proximal cognitions (attitude toward quitting, social support for quitting and perceived smoking behavior in the environment (modeling), self-efficacy expectations, intention to quit). 
The proximal cognitions were measured according to the ASE model of behavioral determinants, a cognitive model which is commonly used to understand and predict health behavior (De Vres et al. 1988; Kok et al., 1991), and is quite similar to the Theory of Planned Behavion (Azen, 1991; Godin \& Kok, 1996). Reliability of all cognitive scales was sufficient (cronbach's $a>60)$ and has been reported elsewhere (Bolman \& De Vries 1998 .

Motivational phases were assessed by using an adaptation of the Prochaska \& DiClemente Stage of Change (SoC) typology (Prochaska \& DiClemente, 1992; Prochaska et al. 1992). In the current paper, stages are referred to as phases of chiange, because the term may be used inappropriately within the SoC concept. The reason is that a genuine stage theory has several characteristics, such as mon-reversibility and invariant sequence, which are not met in the SoC concept (Bandura, 1997). Three alterations to the original typology were necessary to make it fit the situation of cardiac inpatients. First, there were no patients in the maintenance phase, because we only included patients who had smoked in the week prior to admission. Second, patients in the action phase were divided into patients situated in internal or external motivational phases, since patients could have been smoke-free at the time of measurement because they wanted to be, but also because of ward regulations 7 or physical restraints. at the time. A previous study among cardiac inpatients had suggested that this subdivision was necessary (Bolman \& de Vries, 1998). Thirdly, patients in the preparation and contemplation phases were combined because of the small sample size of the latter $(\mathrm{N}=11)$. This resulted in four groups at baseline:

smokers in precontemplation phase, had been smoking since admission and were not planning to quit within six months.

Smokers in contemplation phase, had been smoking since admission and were planning to quit within six months.

Patients in external motwation phase; had not been smoking since admission, but intended to start smoking again as soon as possible.

Patients in internal motivation phase, had not been smoking since admission and were planning to maintain non-smoking.

\section{Analyses}

Baseline differences between the two conditions were tested using chi"square statistics for the categorical and dichotomous variables (gender, educational level, prior admission for CVD, reason for admission, 24-hour attempt to quit in the year prior to admission, motivational phase of change, type of hospital) and T-tests for the continuous variables (age, age of smoking initiation. FTND score, intention to quit, pros and cons of quitting, perceived self-efficacy, social support and modelingl.

in order to determine whether selective loss to follow-up had taken place during the follow-up period, attrition between pretest and posttest was assessed by logistic 
regression analysis, using attrition as the dependent varialale and condition and baseline characteristics as predictors.

The effectiveness of the intervention was tested by logistic regression analysis. Condition and cowariates (age; gender; educational level; prior admission for CVD; reason for admission; FTND score; intention to quit; motivational phase; pros and cons of quitting; modeling; social support; self-efficacy; previous quit attempt; age of smoking initiation, type of hospital) were the independent variables. Covariates were always included to correct for baseline differences and to increase the statistical power by reducing unexplained wariance (Robinson \& Jewell, 1991). Based on previous research among CHD patients (Ockene et al., 1992; Rosal et al., 1998), interactions between 1) reason for admission and condition and 2) motiwational phase and condition were tested. Condition and the covariates were entered first as one block, 10 llowed by the interaction terms Interaction terms were then removed one by one $(\mathbb{P}<.10)$, using a hierarchical backward elimination procedure. Because the intervention was supposed to be superior to usual care (i.e, among others it was based on the results obtained with the MIS), an one-sided test was used to test the intervention effect. As regards the covariates, two-tailed tests were used since the literature was ambiguous about the influence of these factors. Furthermore, since four of the eleven hospitals had se. lected their own treatment instead of being ramdomized to the experimental or control condition, an indicator of self-selection of treatment (yes/no) was constructed. The final logistic regression models were re-run with this indicator and its interaction with treatment included as predictors to check whether the self-selection had influenced the treatment effect or the smoking outcomes.

Logistic regression analysis assumes that the outcomes are statistically independent. However, our design was a nested design, with patients nested under hospitals, which may have led to intra-class correlation (i.e. outcomes in the same hospital are related due to hospital effects on the outcome). Since entire hospitals, rather than individual patients within hospitals, were assigned to treatment conditions, ignoring intra-class correlation would lead to underestimation of the standard error of the treatment effect, too narrow confidence intervals and possibly to type 1 errors (Bryk \& Raudenbusch, 1993; Goldstein, 1995a; Longford, 1995\%. We therefore re-ran oul final logistic regression models with mixed effect logistic regression using EGRET version 1.02.10 (Statistics and Epidemiology Research Corporation, 1993), treating patients nested within hospitals and hospitals as random effects.

\section{Sample Size}

Based on previous research, it was assumed that $40 \%$ of the control patients would quit, against $50 \%$ in the intervention group. For a significance level of .05 (onetailed) and a power of $80 \%$, this implied a sample size of 650 patients. Assuming $20 \%$ dropouts, the sample needed to include 815 smoking patients at baseline. 


\section{Results}

\section{Response and Attrition Analysis}

From November 1995 to May 1997, 891 hospitalized smokers were identiffed. Thirty-eight patients (4\%) refused to participate. Reasons for refusal were not obtained since medical-ethical policies did not allow this. Furthermore, 64 patients $(8 \%)$ had to be excluded because $30 \%$ or more of the baseline questionnaire had not been filled in.

\section{hospitalized smokers $(N=891)$}

control group $(N=458)$

refused to participate $(N=19)^{\text {a }}$ missing baseline data $(N=38)^{a}$

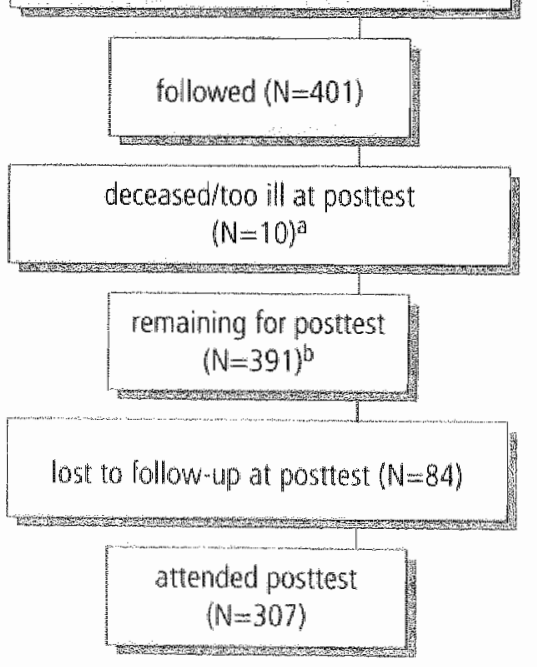

intervention group $(\mathrm{N}=433)$

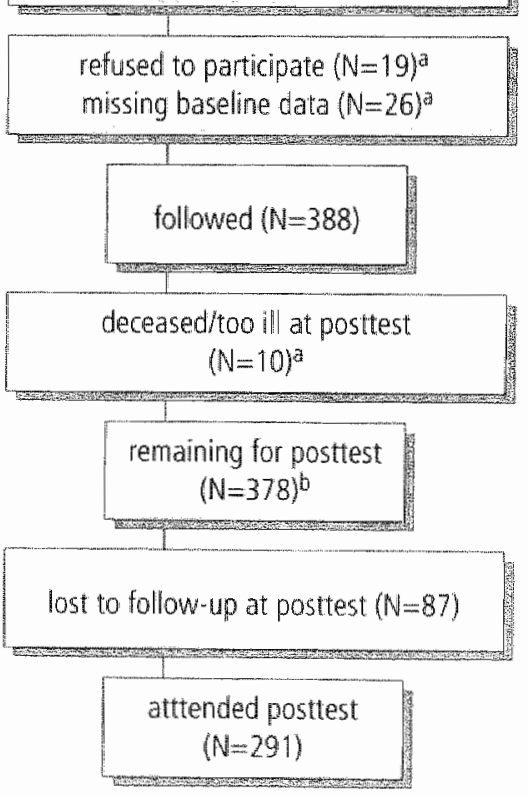

a excluded from all analyses $(N=122)^{\circ}$ included in intention-to-treat anallysis $(N=769)$

Egure 3.2. N of patients

Of the remaining patients $(\mathrm{N}=789), 401(51 \%)$ were in the control group and $388(49 \%)$ belonged to the intervention group (see Figure 3.2). 
Table 3.1 Baseline characteristics for interuention and control cardiac wapatients $(N=789)$

Frior admission for CVD, \%

Edutational level, up to secondary vocational school, $\%^{\mathrm{a}} \quad 86 \%$

Reason for admission. \%

Myocardial Infarction

Angina Pectoris

Unknowntio

Other:

Intention to quit, mean $\pm S D$

$(1=$ wery weak, wery strong $=10$ )

Motivational phase, \%

precontemplation

contemplation

external motivation phase

internal motivation phase

Pros, mean \pm 50 ( $0=$ no pros, $3=$ many pross

Cons, mean $\pm S D \quad(0=$ no cons. $-3=$ many cons $)$

Social support, mean \pm SD

( $3=$ much discouragement, $3=$ much support)

Modeling, mean \pm 50

$(-1=$ everyone smokes, $1=$ no one smokes $)$

Sell-efficacy expectations, mean $\pm S D$

$(-3=$ very difficult, $3=$ very easy $)$

Age at smoking initiation, mean \pm 50

Level of addiction, Fagerström, mean \pm 50

( $1=$ no physical addiction, $10=$ serious addiction)

Prewious quit attemp? \%

Age, mean $\pm 5 D$

Self selection of treatment, \%

Type of hospital (general hospital), \%

Men, \%

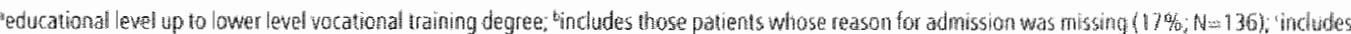

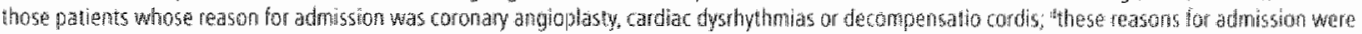

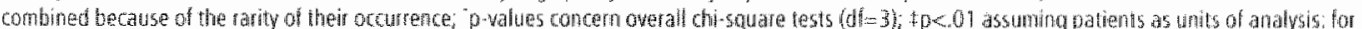

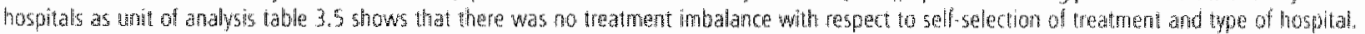

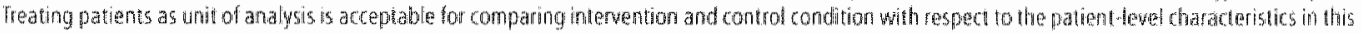
table, but not tor the wo hospital- level characteristics.

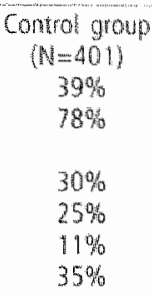

$<.05$

$<.01^{\circ}$

$37 \%$

$16 \%$

$6.36 \pm 2.9<.01$

$10 \%$

$12 \%$

$35 \%$

$3 \%$

$\begin{array}{rrr}1.78 \pm 0.5 & 1.68 \pm 0.6 & <.05 \\ -1.28 \pm 0.7 & -1.34 \pm 0.7 & \text { N.S. } \\ 1.38 \pm 0.9 & 1.04 \pm 1.0 & <.01 \\ 0.09 \pm 0.4 & 0.06 \pm 0.4 & \text { N.S. }\end{array}$

$-1.55+1.3$

$-1.66 \pm 1.3 \quad$ N.S.

$17.2 \uplus 4.5$

$17.1 \pm 4.3$

N.S.

$4.4 \pm 2.4$

N.S.

$55 \%$

N.S.

$57.3 \pm 10.9$

N.S.

$46 \%$

1

$79 \%$

$78 \%$

$78 \%$

N. 5

At the posttest, 10 patients (2\%) in the intevention group and 8 patients $(2 \%)$ in the control group were deceased and therelore excluded from the analyzes. Two control patients were too ill to fill in the questionnaire and were also excluded. Eightyseven (23\%) patients in the intervention group and $84(21 \%)$ in the control group were lost to follow-up. In sum, from pretest to posttest $78 \%(M=598)$ of the remaining 769 
responders were reached (see Figure 3.2). Logistic regression analysis on attrition revealed that patients lost to follow-up were more often from the intervention group $(O R=1.55,95 \%$ confidence interval $(C)=1.04-2.29, p<.05)$, perceived more pros of quiting $(O R=1.52, \mathrm{C}=1.06-2.19, \mathrm{p}<.05)$, had weaker intentions not to smoke after hospital discharge (OR=0.91, $\mathrm{Cl}=0.85-0.99, p<.05)$, perceived fewer non-smokers in their dired environment $(O R=0.48, C l=0.30-0.78, p<.05)$, and were less often in the internal motivation phase $(O R=0.33, C l=0.15-0.73, p<.05)$.

\section{Comparison of Groups at Baseline}

As can be seen in Tabie 3.1, the intervention group contained significantly more patients with a lower level of education, fewer patients who had been admitted earlier for CVD, more patients admitted for a myocardial infarction and less patients for the category 'other' than the control group. Patients in the intervention group also reported significantly stronger intentions to quit smoking, reported more positive consequences of quitting, perceived more social support and were more often in the internally motivated action phase.

\section{Process Evaluation}

With respect to the degree of patient exposure to the intervention components, the process evaluation among patients in the intervention group revealed that $76 \%$ of the patients had received the self-help manual, $65 \%$ had received the brochure, $84 \%$ reported having spoken about smoking cessation with the nurse during their hospitalization and $75 \%$ reported that the cardiologist had assessed their smoking behavior during the aftercare. Patients in the intervention group reported that the conversation(s) with the nurse had helped them to perceive more pros and fewer cons of quitting $(x=7.0 ; 5 D=2.89$; scale range: 1 (not at all) to 10 (to at large extent)). As regards the extent to which the conversation had enabled patients to cope with highrisk situations and to obtain support in their immediate environment (cimilar answer scales were used), patients were less positive, with average scores of 5.7 (SD-3) and $5.9(50=2.99)$ respectively. Self-help materials were read moderately well: patients scored averages of $6.39(S D=3.64)$ and $6.23(S D=3.85)$ for the self-help guide and brochure respectively. Intervention patients also rated their appreciation for the way the issue of smoking had received attention during admission and after discharge. The inhospital attention was given an average score of $7.78(S D=2.68)$, while the aftercare was given a $6.57(50=3.35)$.

Compared to control patients, table 3.2 shows that patients of the interwention group indicated significantly more often that nurses and cardiologists had addressed 
their smoking behavior. They furthermore indicated significanty stronger that the conversations with the nurse had motivated them to make an attempt to quit and had made it easier to sustain such an attempt. The same was reported about the conversation with the cardiologist during the aftercare.

\begin{tabular}{|c|c|c|c|c|}
\hline Results of process & 5 evaluation & & & \\
\hline & $\begin{array}{l}\text { Intervention group } \\
(\mathrm{N}=291)\end{array}$ & $\begin{array}{l}\text { Contiol group } \\
(N=217)^{\circ}\end{array}$ & $x^{2}$ test $/$ test & $p<$ \\
\hline $\begin{array}{l}\text { Yes, the nurse has spoken with me about } \\
\text { smoking cessation during my hospital admission }\end{array}$ & $84 \%(n=284)$ & $49 \%(n=184)$ & $x^{3}(1)=68.04$ & .0001 \\
\hline $\begin{array}{l}\text { Yes, the cardiologist addressed my smoking } \\
\text { habits during the outpatient dinic vishl }\end{array}$ & $75 \% \quad(n=278)$ & $67 \%(n=179)$ & $x^{2}(1)=4.07$ & .05 \\
\hline $\begin{array}{l}\text { Has the conversation with the nurse motivated } \\
\text { you to make an altermpt to quit? }\end{array}$ & $6.19(n=243)$ & $5.23(n=159)$ & $1(400)=\cdot 2.89$ & .01 \\
\hline $\begin{array}{l}\text { Has the conversation with the nurse made it } \\
\text { easier for you to sustain your artempt to quit? }\end{array}$ & $6.34(n=239)$ & $5.38(n=152)$ & $1(389)=-2.77$ & .01 \\
\hline $\begin{array}{l}\text { Has the corversation with the cardiologists } \\
\text { motivated you to make an attempt to quit? }\end{array}$ & $6.27(n=209)$ & $5.59 \| n=148$ & $t(355)=-1.90$ & N.S. \\
\hline $\begin{array}{l}\text { Has the conversation with the cardiologist made } \\
\text { it easier for you to sustain your attempt to quit? }\end{array}$ & $6.41(n=210)$ & $5.45(n=155)$ & $(363)=-2.80$ & .01 \\
\hline
\end{tabular}

\section{Effectiveness of C-MIS}

An "intention-to-treat" procedure treating patients lost to follow-up (dropouts) as failures, i.e., as posttest smokers is often recommended to evaluate treatment effects because it is not affected by self-selection issues (Hajek, 1994; Lando, 1993; Lichtenstein \& Glasgow, 1992, Windsor, Boyd, \& Orleans, 1998). This option might, however, be too conservative in studies with high dropout rates. Therefore, and in line with several other studies among cardiac inpatients, we performed one analysis without dropouts and one with dropouts regarded as smokers (Rigotti et al., 1994; Taylor et al., 1990).

Logistic regression analysis excluding dropouts revealed no interaction effects ( $p$ values $>.25$ ). Table 3.3 shows the final logistic regression model with a significant intervention effect on point prevalence abstinence $(O R=2.11,90 \% \quad \mathrm{Cl}=1.50-2.98$, $p<.001$, one-tailed). The unadjusted OR for the intervention effect was slightly higher $(\mathrm{OR}=2.48,90 \% \mathrm{Cl}=1.76-3.50, \mathrm{p}<.001$, not presented in the table). Point prevalence abstinence was furthermore significantly associated with the following baseline variables (Table 3.3): being an internally motivated actor, having high self-efficacy expectations, being admitted for a myocardial infarction and making a first attempt to quit. 


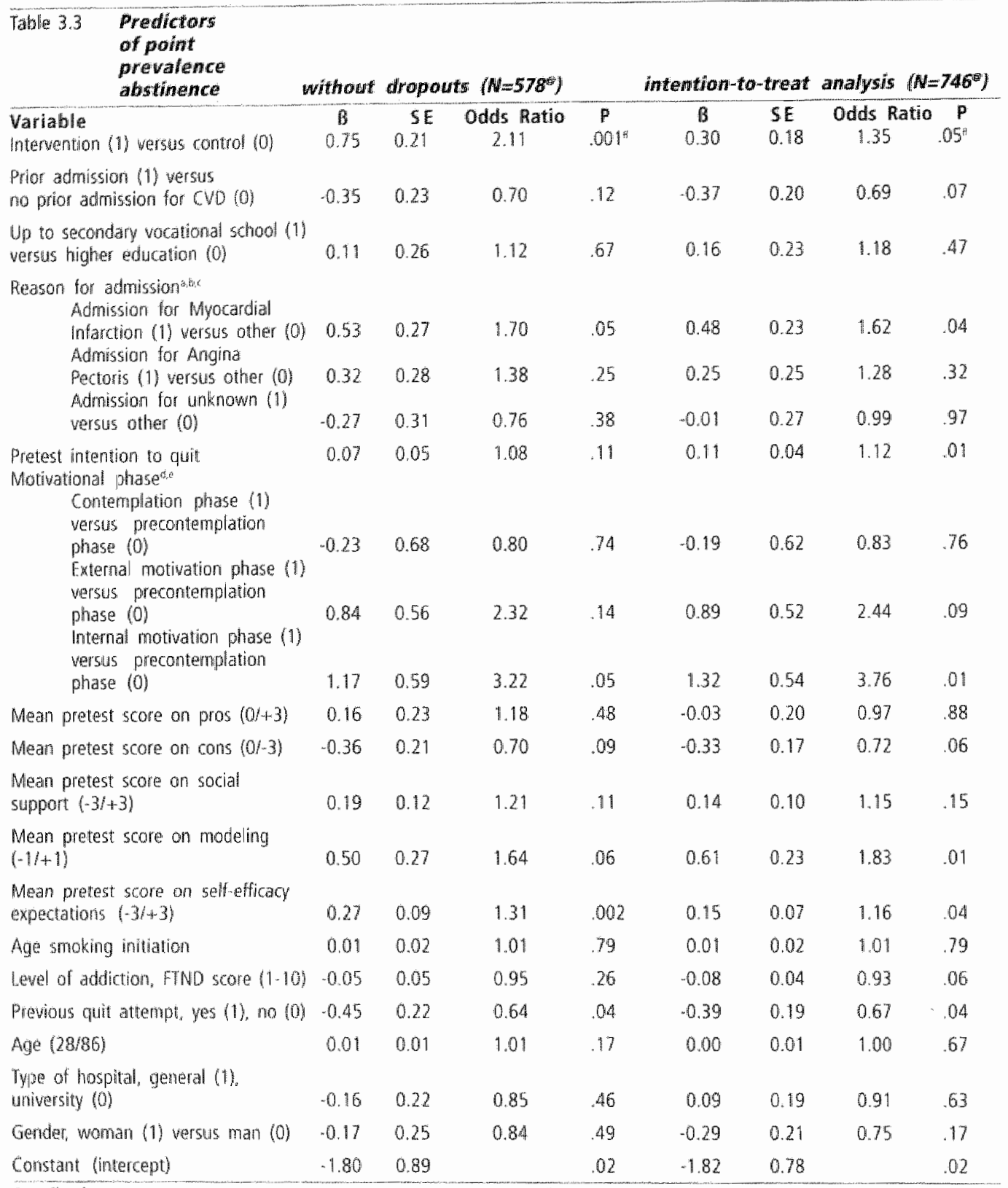

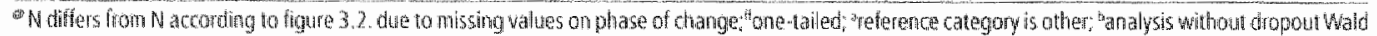

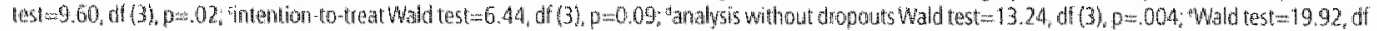

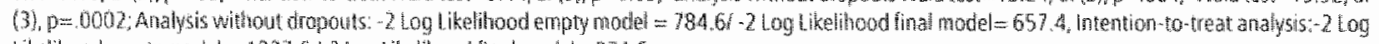

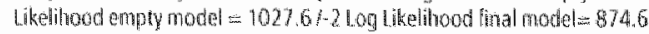




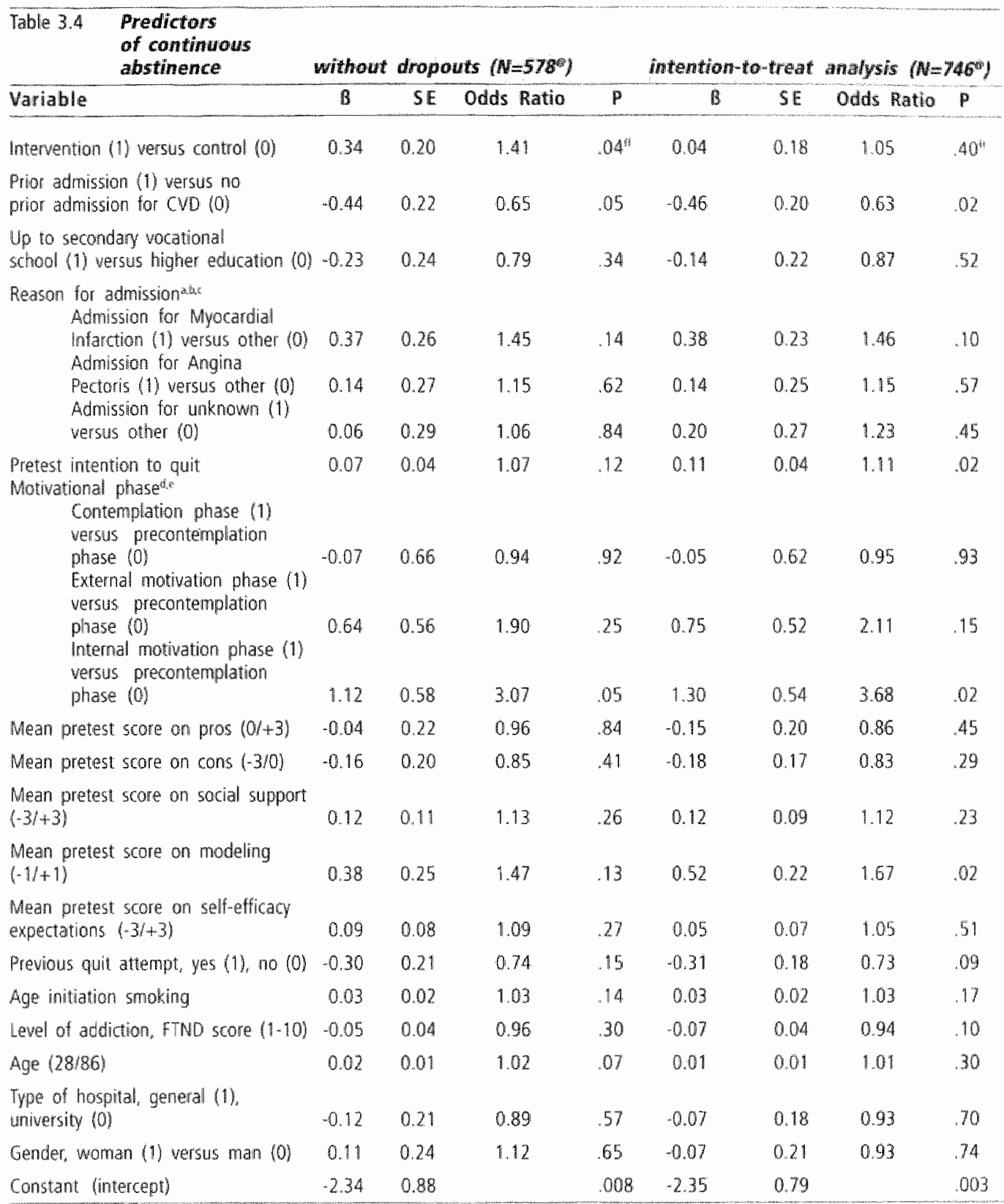

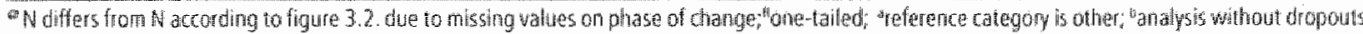

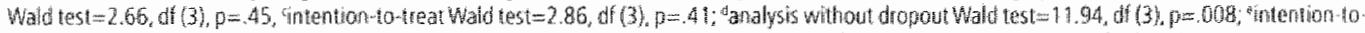

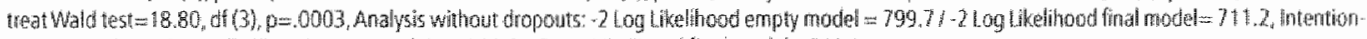

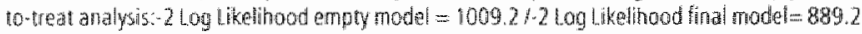


With regard to continuous abstinence, no interaction effects were found either ( $p$-walues $>79)$. As table 3.4 shows, a significant intervention effect was found $\angle O R=1.41,90 \% \mathrm{Cl}=1.02-1.95, p<.05$, one-tailed in the regression model adjusted for covariates. Again, the unadjusted $\mathrm{OR}$ for the intervention effect was higher (OR $=1.95$. $90 \% \mathrm{Cl}=1.45-2.61, \mathrm{p}<.001$, not presented in the table). Continuous abstinence (Table $3.4)$ was associated with not having been admitted for CVD before $(p<05)$ and with being an internally motwated actor $(p<.001)$.

\section{Table 3.5 smoking cessation per hospital $(\%)$}

\section{dropout (\%)}

\section{without dropouts}

continuous abstinence

\section{intention-to-treat}

point prevallence continuous absinence abstinence

\section{intervention condition.}

hospital $1^{\text {an }}(\mathrm{H}=80 \mathrm{0})$

hosplital $2^{\circ}(\mathrm{n}=162)$

thospital $3(\mathrm{~N}=40)$

hospital $4(\mathrm{~N}=48)$

hospital $5(N=47)$

$\begin{array}{ll}24 & 69 \\ 19 & 6 \\ 30 & 8 \\ 25 & 6 \\ 30 & 6\end{array}$

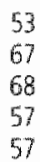

93

67

68

57

57
52

40

$60-2-10$

$52 \quad 42$

$40 \quad 32$

control condition:

hospital itis $(\mathrm{N}=117)$

hospital $2^{2}(N=63)$

hospital $3^{\circ} \quad(N=62)$

hospital $4(1 \mathrm{~N}=79)$

hospital $5(N=64)$

hospital $6(\mathrm{~N}=7)$

mean $\%$ trve interwention hospitals 26

mean $\%$ six control hospitals

$\begin{array}{ll}24 & 5 \\ 20 & 30 \\ 21 & 39 \\ 16 & 5 \\ 33 & 5 \\ 0 & 67 \\ 26 & 7 \\ 19 & 50\end{array}$

54
30
39
56
51
67
71
50

51
24
37
52
47
50
60
44

stelfosedected hospital; "university hospital.

Logistic regression analysis according to the intention-to-treat procedure and treating dropouts as smokers also revealed no interaction effects on both outcomes ( $p$-values $>$.41). A significant intervention effect on point prevalence abstinence $(O R=1.35,90 \%, C \mid=1.01-1.81, p<.05$, one-tailed) was found if covariates were included (see Table 3.3). The unadjusted of for intervention effect was $1.95(90 \% \mathrm{Cl}=1.45-2.61$, $p<.001$, not presented in the table). Covariates associated with point prevalence abstinence were similar to those found in the analysis excluding dropouts, with the exception of a significant association between modeling and the outcome and intention and the outcome. For continuous abstinence (see Table 3.4), no significant intervention effect was found if covariates were included $\angle O R=1.05,90 \% \mathrm{Cl}=0.78-1.40, p=.40$, onetailed). The unadjusted $O R$ was, however, significant $(O R=1.65,90 \% \mathrm{Cl}=1.23-2.22$, $p<001$, not presented in the table). Effects of covariates were similar to those in the analysis without dropouts, except for the significant associations of the outcome with intention and modeling.

Forced entry of the indicator for self-selection of treatment condition by the hospital at hand and its interaction with treatment into the final models (see method sec- 
fion) suggested that self-selection had neither intuenced the treatment effects (as interaction effects; $p$-values $>43$ ) nor influenced the smoking outcomes (as main offects; $p$-values $>.22$ after eliminating the interaction from the model). Magnitude and significance of the treatment effects were not substantially different from the regres. sion analyses without the indicator for self-selection included.

A check of the assumptions for lagistic regression gave satisfactory results for all outcome variables reported. Comparison of the logistic regression output with re-analysis of the final models using random effect logistic regression in EGRET (Statistics and Epidemiology Research Corporation, 1993) did not show random hospital effects for the outcome variables (all p-values > 21). Magnitude and significance of the treatment effects were comparable to those obtained in SPSS.

Detailed insight into the quitting behavior in each individual hospital can be deriwed from table 3.5 . The table shows that the intervention hospitals generally showed higher point prevalence and continuous abstinence cessation rates than the control hospitals, though the differences were substantially smaller in the intention-to-treat analysis.

\section{Discussion}

The process evaluation data on program exposure showed that most patients in the intervention group had indeed received the intervention. Patients in this group also reported that the conversations with the nurse had motivated them to quit smoking. They were, however, less pasitive about the guidance they had experienced regarding social support and coping, which suggests that nurses need to improve their performance in this respect. Previous studies also reported that nurses experienced difficulties in helping patients to cope with high-risk situations (Kviz et al., 1995; Nagle et al., 1999). Therefore, it seems desirable to improve the nurses' training, for example by supplementing it with feedback sessions to review and improve skills acquisition. Several studies (Miller et al., 1997; Ockene et al., 1992: Taylor et al., 1990) have suggested that nurses' training should include follow-up, which was not posible in the present study due to financial and time constraints.

Significant intervention effects were found on both outcomes when dropouts were not included. However, the intention-to-treat analyses, which included dropouts as smokers, only showed a significant effect on point prevalence abstinence. These results were probably not distorted by baseline differences, nor by possible covariates, since they were accommodated for by induding them in the regression model. Evidence that no distortion was introduced can also be found in the fact that the unadjusted odds Ratios for the intervention effect were higher and significant for all outcomes. No evidence was found that the differences in the randomization procedure, resulting in self- 
selection by four of the eleven hospitals, influenced the treatment effect (as an interaction effect) or the smoking cessation outcomes (as a main effect). Furthermore, the random effect logistic regression analyses did not show a random hospital effect, and confirmed the results of the SPSS analyses. Intervention effects were not modified by the two patient characteristics that were suspected to interact with treatment, since interactions were absent.

In sum, considering the intention-to-treat results as the most appropriate to evaluate the effectiveness (due to the substantial dropout rate and its relation to treatment and some baseline characteristics), it can be concluded that the intervention produced a small but significant intervention effect on point prevalence abstinence. It is conceivable that greater effects can be reached. The present results were obtained under conditions with moderate intervention coverage. Moreover, nurses' efforts in helping patients to obtain social support and to cope with high-risk situations were not always successfully employed. Finally, the intention-to-treat results reflect the most conservative effect estimation.

In contrast to our intention-to-treat intervention effects, three other studies among cardiac inpatients failed to find significant differences in (intention-to-treat) point prevalence abstinence after four (Rigotti et al., 1994) and six months (Johnson et al., 1999; Orleans et al., 19901. Ockene and colleagues (1992), however, reported intervention effects on (intention-to-treat) point prevalence abstinence that were comparable to those found in the present study, though they were measured after six months and only for patients admitted with severe myocardial infarction. Two other studies reported stronger (intention-to-treat) intervention effects than those found in the present study, using one year follow-up measurements (Miller et al., 1997; Taylor et al., 1990). Taylor's study, however, concerned only myocardial infarction patients, whereas our intervention also aimed at patients admitted for less severe complaints such as angina pectoris, who are less likely to quit smoking than myocardial infarction patients (Ockene et al., 1992; Van Berkel et al., 1999a). All other studies referred to here involved more extensive inteventions than ours. Furthermore, the Odds Ratios we found were comparable to those reported in the Cochrane Review on the efficacy of nursedelivered interventions in general (Rice \& Stead, 1999). This review, however, only included randomized trials with a follow-up period of at least six months. The effects we found were less strong than the twelve-months effects of the MIS, on which our inter. vention was based (Pieterse, 1999; Pieterse et al., 1994).

Our process evaluation revealed that the implementation of the protocol and the training of nurses need to be improved, as does the protocol used. Miller and colleagues (1997) found that repeated telephone support (four calls) after discharge increased the cessation rate significantly after one year. Hence, we would recommend intensifying the aftercare with several follow-up telephone calls. Furthermore, we agree with recommendations to include nicotine replacement therapy (NRT) as a formal aid in future interventions for cardiac patients (Fiore et al., 2000); this was not 
included in the intervention in the present study. Finaly, disease-prone personality factors such as state-anxiety and depressive symptoms, which often chatacterize the target population (Hemingway \& Marmot, 1999; Huijbrechts et al. 1996; Mckenna \& Higgens, 1997), may need greater attention in the intervention. CHD patients with anxiety and depressive feelings have shown greater persistence with regard to smoking (Huijbrechts et al., 1996). Although it is a challenge to intervene successfully on these factors in a minimal intervention like that used in the present study, efforts have to be made to find out how smoking patients who suffer from psychological problems can be approached successfully by using an additional counseling module for this group.

In line with previous findings (Glasgow el al. 1991; Ockene et al. 1992; Orleans et al. 1990; Rigotti et al., 1994; Rosal et al., 1998; Taylor et al., 1990), smoking cessation was associated with the reason for admission, previous admission for CVD, intention to quit, modeling, self-efficacy and the motivational phase that patients were in at the pletest.

The present study is subject to a number of limitations. First, although a full randomized control trial was planned at the start of our study in order to strengthen the internal validity of the study (Murray, Hannan, \& Zucker, 1989) and to awoid betweengroup contamination (Sackett, Haynes, \& Tugwell, 1988), the randomization procedure failed for four hospitals. In depth-analyses showed, however, that similar treatment effects were found for patients in the self-selected hospitals and the randomized hospitals. Second, attrition was high and selectivity occurred. Because the selectivity concerned treatment condition and baseline characteristics which were also independently related with smoking cessation, the effects found in the analyses wit hout dropouts might have been overestimated. The selective dropout may also have lowered the external validity. The high attrition rate was partly caused by patients changing their home addresses without informing us. Utilization of a tracking system based on hospital records (which was not done in the present study) is therefore recommended. Telephone fallow-up assessments are also recommended, since this procedure may result in attrition rates of 10\% or less (Miller et al, 1997; Ockene et al. 1992; Rigotti et al., 1994; Taylor et al., 1996). Third, despite our elforts to obtain a representative sample of smoking cardiac inpatients, the long period needed to include sufficient numbers of patients suggests that not all smoking patients were approached. Nurses confirmed this, mentioning time constraints and forgetfuness as the most important causes. Hence, the patien sample may not be representative.

Fourth, no procedures were used to validate self-reported smoking behavior. Patients may have under reported or falsely reported their smoking behavior. However. several intervention studies in the general population (Glasgow, Mullooly, Vogt. Stevens, \& el al., 1993; Kotke et al., 1988; Murray, Connett, Lauger, \& Voeker, 1993) as well as among CHD patients (Van Berkel et al. submitted b; Miller at al. 1997; Ockene et al., 1992; Taylor et al., 1990) have reported that treatment groups are not more 
mely to misepresent their smoking behavior than controls. Therefore, we assume that any bias that nay have occurred did not significantly affect the differences found between the groups, Moreover, some researchers (Hansen, Malotte, \& Fielding, 1985; Lichvenstein \& Glasgow, 1992) have reported that self-reports are accurate when anonymity and confidentiality are assured and an identification coding system is used. which was the case in the present study. Biochemically valdated studies among patients with coronany artery diseases found that self-reports were walid for this specific population (Rice et al., 1994; Sirota et al., 1985).

A fifth limitation concerns the power of the study. It is unclear whether the study had enough power to detect interactions in the regression analysis, since we did not include them in our power calculations. Furthermore, when planning the sample size of the study, the nesting of patients within hospitals was ignored. This may have led to an underestimation of the standard error of the treatment effect and thereby also of the sample size needed. However, the data analysis showed neither hospital effects nor underestimation in analyses that ignored the nesting. 


\section{A MINIMAL-CONTACT INTERVENTION FOR CARDIAC INPATIENTS: LONG-TERM EFFECTS ON SMOKING CESSATION*}

\section{Abstract}

Background. This study examined the one-year effectiveness of a minimal-contact smaking cessation intervention for cardiac inpatients.

Methods. The multi-center study included cardiac inpatients who had smoked prion to hospitalization. A Pretest-Posttest quasi experimental design was used, in which hospitals were assigned to the intervention or control group. Patients' experimental condition depended on the hospital they were assigned to. The design was partially randomized: 4 of the 11 hospitals selected the experimental condition themselves $(2$ experimental, 2 control), while the remaining 7 hospitals were randomly assigned. The experimental group consisted of patients of 5 hospitals $(N=388)$. Patients of 6 other hospitals served as the control group ( $N=401)$. The intervention included stop-smoking advice by the cardiologist, brief counseling by the nurse, the provision of self-help materials and aftercare by the cardiologist.

Results. Logistic regression analyses controlling for baselime differences and covariates showed significant intervention effects on point prevalence and continuous abstinence. Intention-to-treat analysis, however, failed to corroborate these effects. The study also showed that the outcomes were not significantly related to the way hospitals were assigned to the experimental condition.

Conclusions. Although the results are encouraging, there was no unequivocal evidence for the effectiveness of the intervention. Efforts should be made to improve the intervention, especially the aftercare.

*Submitted as: Bolman, C, De Vries, H. Van Breukelen, G. A minimal contact intervention for cardiac inpatients: long-term effects on smoking cessation. 


\section{Introduction}

Smoking cessation after the development of coronary heart disease (CHD) improves a patient's prognosis more than any other treatment (Cameron, Davis, \& Rogers, 1995; Deckers et al., 1994; Mehta \& Eagle, 1998; Smith, 1997; U.5. DHHS, 1990). It is therefore desirable to assist cardiac inpatients in attempts to stop smoking. The hospital setting presents an excellent opportunity for cessation interventions. During hospitalization, cardiac patients are more aware of their personal vulnerability to the dangers of smoking, and their serious illness makes them more motivated to quit and more receptive to interventions (Emmons \& Goldstein, 1992; Miller et al., 1997; Orleans et al., 1993b; Orleans \& Ockene, 1993; U.S. DHHS, 1996). Moreover, patients have often been smoke-free for some days and may therefore be more wiling to continue to refrain from smoking. Several randomized controlled studies have examined the efficacy of in-hospital smoking cessation interventions for patients with CHD (Miller et al., 1997; Ockene et al., 1992; Rigotti et al., 1994; Rosal et al., 1998; Taylor et al., 1990; Taylor et al., 1996). One year posttest measurements in these studies revealed point prevalence abstinence rates ranging from $35 \%$ to $61 \%$ in experimental groups, compared with rates ranging from $28 \%$ to $54 \%$ in control groups. However, these interventions were characterized by at least one hour of behawioral counseling and several brief follow-up contacts after discharge, which made them very laborious.

A working group of the Netherlands Society of Cardiovascular Nursing who focused an smoking cessation guidance, however, indicated that cardiac nurses could only spend a maximum of 30 minutes on a smoking cessation intervention. Therefore, a minimal-contact smoking cessation intervention was developed and tested, an adaptation of the Miminal Intervention Strategy (MIS) that had already proved its efficacy in general practice (Pieterse, 1999; Pieterse et al., 1994). The adapted wersion (called C-MS), requires a time investment of $15-30$ minutes. A search of the literature revealed no evaluation of such a minimal-contact intervention for hospitalized CHD patients.

The C-MIS was developed in order to stimulate smoking patients to quit and to prevent relapse among patients who have not smoked since hospital admission. Since the efficacy of the MIS had already been tested, the present study focused on assessing the effectiveness of the protocol in actual clinical circumstances (Windsor et al., 1994). For this purpose, the behavioral effects of the intervention after twelve months were assessed, as well as the predictors of quituing. 


\section{Methods}

\section{Patient Recruitment and Design}

Twenty-one hospitals, were approached for participation in the study by contacting the managers of the cardiac wards. Ten hospitals refused to participate for various reasons, induding a ward merger, current participation in other time-consuming studies, staff turnover and personnel shortage. Initially, the remaining eleven hospitals were to be randomly assigned to the experimental and control conditions. However, two hospitals only wanted to serve as controls, whille two other hospitals only wanted to be inwolwed in the experimental condition. This situation resulted in self-selected assignment of four hospitals and random assignment of the remaining seven hospitals. Five hospitals served as the experimental group and six as the control group. Assignment of patients consequently depended on the hospital to which they had been admitted. Hospitals rather than patients were thus used as the units of assignment in order to avolid between-group contamination (Sackett, Haynes, Guyatt, \& Tugwell, 1991), since the latter would threaten the internal validity of the study (Murray et al., 1989). The units of analysis were patients, nested within hospitals.

The study included three university and eight general hospitals. The number of beds in the hospitals ranged from 440 to 1174 , while the number of beds on the coronary care wards ranged from 8 to 41 . The cardiac treatments offered in the hospitals were comparable.

From November 1995 to May 1997, all patients who had smoked in the week prior to admission to the cardiac wards were recruited by ward nurses. During the enroll. ment period, patients' smoking behavior in the seven days prior to admission was routinely assessed by nurses at intake. In emergency admissions, the patients' smoking behavior was assessed later, but within 96 hours from admission. Every day during the briefing at shift change it was communicated whether a patient was eligible for inclusion in the study or whether his or her smoking behavior still needed to be assessed. Internal program co-ordinators coordinated and supervised the process of inclusion. These co-ordinators and nurses were explicitly instructed to ask all patients who were eligible for inclusion, and to register refusals. Nurses informed patients about the study and asked them to wolunteer for a project on smoking cessation, including the possibility of being a contral group member. Nurses also handed patients a letter containing the same information as that prowided oraly by the nurse. Patients who were willing to participate had to give informed consent. Since nurses in the experimental condition could not be blinded for the treatment status (as they implemented the inter. vention), they were explicitly instructed by the researcher and internal program coordinators not to inform patients about the content of the control and experimental treatments. In the control group, only the internal program co-ordinators were infor- 
med about the treatment condition to which their ward had been allocated. In order to blind the control ward nurses for the condition to which their ward had been allocated, the co-ordinators were instructed to give their ward nurses only a minimum of information about the study design and not to inform them about the treatment condition to which the ward had been ahlocated.

A pretest was conducted by means of a questionnaire administrated by ward nurses at the hospital atter patients had signed their informed consent forms; posttests were conducted by means of questionnaires that were maled to patients' home addresses at three and twelve months after hospitalization. Results of the posttest after three months have been reported elsewhere (Bolman, De Vries, \& Van Breukelen, in press).

\section{Intervention}

Control patients received the usual care, implying that no systematic attention was given to smaking. The experimental group received C-MIS, which consisted of stop-smoking advice by the cardiologist, followed by 15-30 minutes of standardized individual counseling and the provision of self-help materials by the ward nurse, and aftercare by the cardiologist. Ward nurses informed the cardiologists which patients needed cessation advice. The cardiologist provided this advice when seeing a patient during his daily ward visit. After this advice, the nurse visited the patient and provided more in-depth counseling, which was attuned to the patient's phase of change.

Counseling by the nurse included the following: standardized assessment of the patients" smoking behavior, their degree of addiction and their motivation to quit; addressing the positive and negative consequences of quitting, especially in the case of patients who were unmotivated to quit; addressing the barriers to cessation (expected problems and high-risk situations) and patients' self-efficacy expectations concerning smoking cessation; and encouraging patients to set a date for quitting.

Aftercare was provided by the cardiologist four to six weeks after hospital discharge, during the first check-up at the cardiology outpatient clinic. He or she addressed various aspects of smoking, depending on the new assessment he or she had made of the patient's smoking behavior. To remind the cardialogist, patients' hospital charts were stamped with a prompt by the nurse and included an intervention card briefly reporting the content of the in-hospital part of the interwention. This card was developed specifically for the present study and was used by nurses to record what aspects of the intervention had been completed. They recorded, for example, whether the cardiologist had advised a patient to quit. In addition, a copy of the intervention card and a letter in which the protocol was explained were sent to the patient's general practitionel: The letter asked the general practitioner to pay attention to the patient's smoking behavior if he or she visited the general practice after discharge from the hospital. 
The intervention incorporated the principles of Bandura's 50 cial Leaming Theory (Bandura, 1986) combined with the ASE model (De Vries et al., 1998), theories on relapse prevention (Marlatt \& Gordon, 1985), the Stage of Change Theory (Prochaska \& Di. Clemente, 1983; Prochaska et al., 1992) and motivational interviewing strategies (Miller \& Rollnick, 1991).

\section{Training on the Use of C-MIS}

Prior to the start of the study, the nurses in the experimental group received a standardized two-hour training from the research team. The training included informa* tion on the rationale of and the theory behind the intervention. The steps of the protor col were explained and practiced in roleplays. In addition, every ward received a training manual with instructions on how to implement the pratocol and a poster with a description of the protocol, while every nurse received a pocket-size reminder chart including an overwew of the protocol and standardized messages on the pros of quitting and on coping with highth-risk situations. To ensure quality control, each ward appointed two internal program co-ordinators (see also the previous subsection) who supervised the interventions and to whom nurses could turn with questions. Moreover, meetings between these co-ordinators and the researcher were held every three months to discuss progress, difficulties and experiences. Cardiologists and general practitioners received the protocol and written instructions on how to use it. Cardiologists also received instructions from the internal program co-ordinators.

\section{Measurements}

The posttest assessed point prevalence abstinence by asking: "Have you smoked during the last seven days, even if only ane puff?" (yes/no). Secondly, continuous abstnence was assessed by asking: "Have you smoked since your hospital discharge, even if only one puff?" (yes/no). The words 'ewen if only one puff" were included in the question to indicate that absolute abstinence from smoking was required for both outcomes to be categonized as abstinent. Financial restraints precluded biochemical validation, so only self-reports were used. To enhance the validity of these self-reports, a bogus pipeline procedure was used (Hansen et al., 1985; Murray et al., 1993; Velicer et al. 1992). According to this procedure, patients were informed that they could be invited for a saliva test to validate their self-report. Simultaneously, they were asked to give permission for such a test. 
Baseline measurements included age, gender, educational level, prior admission for CVD, reason for admission, 24-hours quiting attempts in the year prior to admission, age of smoking onset, hospital type and nicotine dependence. The latter was measured by means of the Fagerstrom Test for Nicotine Dependence (FND), which includes the dally number of cigarettes (Heatherton et al. 1991). Cognitive factors measured at baseline included an assessment of perceived positive (pros) and negative consequences (cons) of quitting, social support, modeling, self-efficacy expectations, intention to quit and motivational phases of behavioral change. Most cognitive factors were measured according to the ASE model of behavioral determinants (De Vries et al., 1988; De Vries \& Mudde, 1998). Reliability of all cognitive scales was sufficient (Cronbach's $\alpha>60$ ), as has been reported elsewhere (Bolman \& De Vries, 1998). Motivational phases were assessed by using an adaption of the Stage of Change typology (Prochaska et al, 1992). Some alterations were necessary to make this typology fit the situation of Dutch cardiac inpatients, since some patients could be forced into action by non-smoking regulations in force at the ward or by physical constraints, while others acted because they were motivated to quit. Therefore, a distinction was made between externally and internally motivated actors. Furthermore, the study did not include maintainers, since only patients who had smoked during the week prior to admission were included. Finally, the category of preparators was combined with that of contemplators, due to the small group size $(\mathbb{N}=11)$. These alterations resulted in the following motivational phases at baseline:

Smokers in the precontemplation phase had smoked since hospital admission and were not planning to quit within sik months.

smokers in the contemplation phase had smoked since hospital admission and were planning to quit within six months.

Externally motivated actors hald been abstinent since hospital admission due to ward regulations or because of physical constraints, and intended to start smoking again as soon as possible.

Internally motivated actors had been abstinent since hospital admission and intended to continue to refrain from smaking (Bolman \& De Vries, 1998).

\section{Analyses}

To check the comparability between the groups at baseline, chi-square statistics were used for categorical and dichotomous variables (gender, educational level, prior admission for CVD, reason for admission, 24 -hour quitting attempts in the year prior to admission, motivational phase, hospital type), while T-tests were used for continuous variables lage, intention to quit, pros, cons, self-efficacy expectations, sociall support, modeling. FTND score, age of smoking onset). 
In order to determine whether the follow-up population showed selective loss to follow-up", attrition between pretest and posttest was assessed by logistic regression with attrition as the dependent variable and baseline characteristics as predictors.

The effect of the intervention was tested by means of logistic regression analyses on point prevalence abstinence and continuous abstinence. For both outcomes we performed two types of analysis, one excluding patients lost to follow-up and one regarding patients lost to followiup as smokers (intention-to-treat procedina). Pretest variables were always included as covariates to correct for baseline differences and to increase the statistical power by reducing unexplained variance (Robinson \& Jewell. 1991). Furthermore, since four of the eleven hospitals had selected their own treatment instead of being randomized to the experimental or control condition, a dummy coded indicator of self-selection of treatment was constructed as afi additional covariate to correct for self-selection in the analyses. The 'treatment' factor and the covariates were entered first as one black, followed by possible interactions between (1) treatment and reason for admission, (2) treatment and motivational phase and (3) treatment and self-selection of treatment. The rationale for the first two interactions was derived from the literature (Ockene ett al., 1992; Rosal et al., 1998). The last interaction assessed whether the treatment effect differed between the self-selected and randomized hospitals. To avoid the problem of multiple testing, we did not test for any other interactions. Non-significant interaction terms were removed using a backward elimination procedure $(\alpha=10$, two-tailed). One-tailed test statistics $(\alpha=.05)$ were used to test the treatment (intervention) effect because it was hypothesized that the intervention would be superior to usual care. Cowariates were tested with two-talled significance ( $\alpha=.05$ ).

Logistic regression assumes that outcomes are statistically independent. However, this study had a nested design with patients nested within hospitals, which could lead to intra-class correlation. Since entire hospitals, rather than individuals within hospitals, were assigned to treatment conditions, ignoring intra class correlation might cause underestimation of the standard error of the treatment effed, as well as too narrow confidence intervals and possibly type 1 errors (Bryk \& Radenbusch, 1993; Goldstein, 1995b; Longford, 1995). Therefore, final logistic regression models were re-run using EGRET random effect logistic regression (Statistics and Epidemiology Research Corporation. 1993), treating patients as nested within hospitals and hospital as a random effect.

Since the regression analyses of treatment on abstinence was adjusted for all ban seline patients variables recorded, we were also able to examine which patient characteristics were predictive of quitting, while controlling for treatment $(\alpha=.05$. two-tailled). This procedure was carried out sepallately for the two outcome variables (point prevalence abstinence and continuous abstinence), with and without regarding patients lost to lollow-up as smokers. 


\section{Results}

\section{Response and Attrition}

We identified 891 hospitalized smokers. Registration by ward nurses of patients who were not willing to participate showed that 38 patients $(4 \%)$ refused. Reasons for refusal could not be obtained since medical ethics policies did not allow this. Furthermore, 64 patients $(8 \%)$ were excluded because the baseline questionnaire had not been filled out. Of the remaining patients $(\mathbb{N}=789)$, $401(51 \%)$ were control group members, while $388(49 \%)$ belonged to the experimental group.

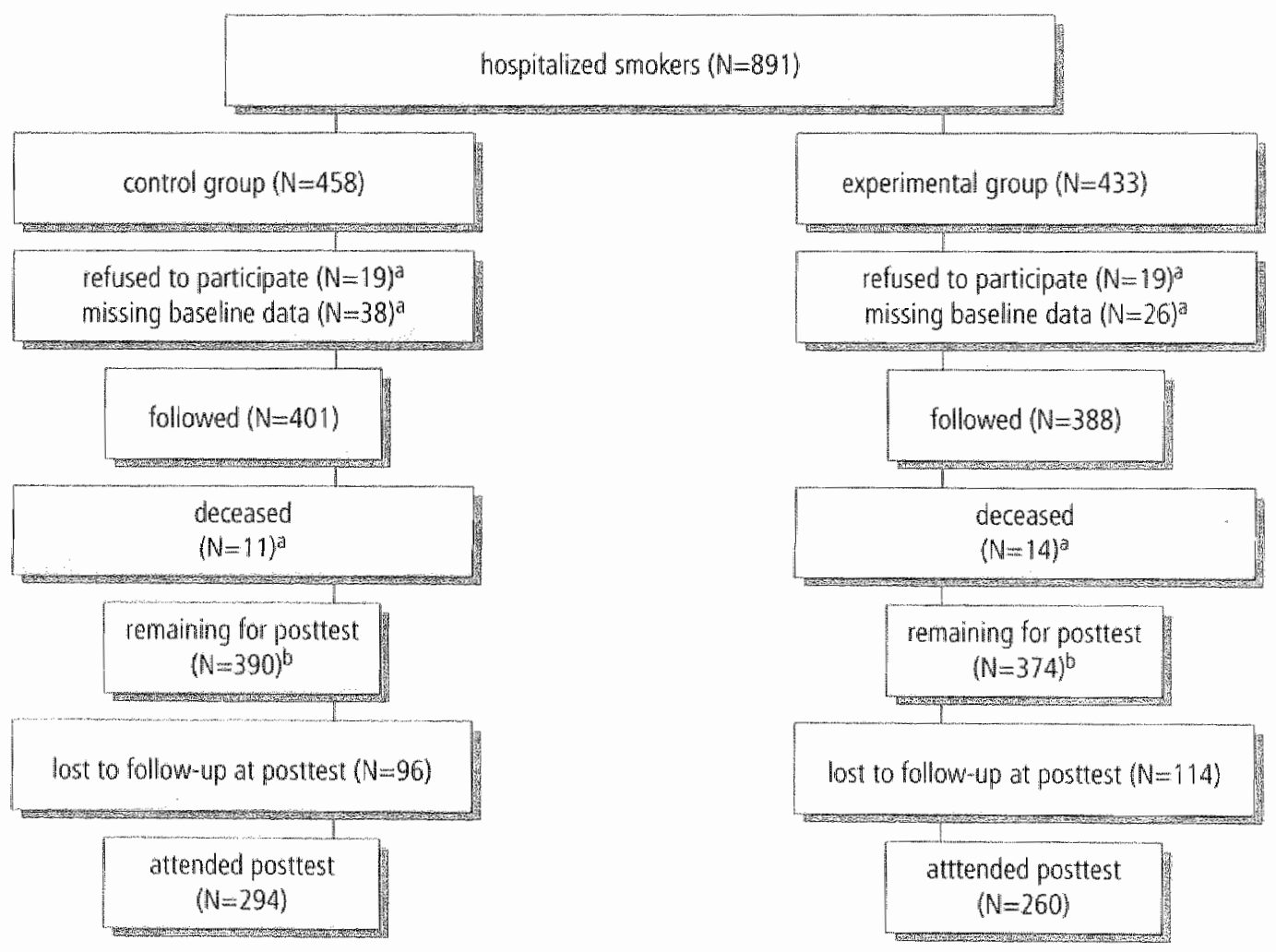

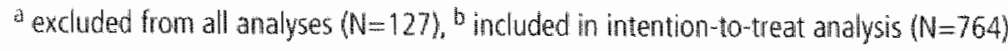

Egure 4.1. Numbers of patients. 
As shown in figure 4. 1, fourteen experimental patients (4\%) and eleven control patients (3\%) were deceased after one year and therefore excluded. One hundred and fourteen experimental patients (30\%) and 96 control group members $(25 \%)$ were lost to follow-up, resulting in a study sample of 554 of the 764 living responders $(73 \%)$

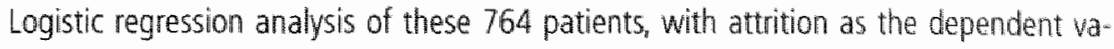
riable and baseline variables and treatment as predictors, revealed that patients lost to follow-up were more often from the experimental group (odds ratio $(O R)=1.81,95 \%$ confidence interval $(\mathrm{Cl})=1.26-2.61, \mathrm{p}<.01)$, had made more attenpts to quit prior to the study $(\mathrm{OR}=1.52,95 \% \mathrm{Cl}=1.06-2.18, \mathrm{p}<.05)$, had experienced more pros of quitting at the pretest $(\mathrm{OR}=1.44,95 \% \mathrm{Cl}=1.04-1.98, p<.05)$, had experienced fewer non-smokers in their environment (modeling) $(O R=0.48,95 \% \mathrm{Cl}=0.30-0.75, p<.01$ ) and had weaker intentions to quit $(O R=0.86,95 \%, C l=0.80-0.92, p<.001)$.

\section{Baseline Characteristics}

We found a number of baseline differences between the experimental and control groups (see Table 4.1). An overall chi-square test showed a significant difference in the reasons for admission. Although the groups differed only slightly with respect to admission for myocardial infarction and angina pectoris, substantial differences were found in the two remaining categories. Motivational phase also differed significantly between the groups. Most importantly, the experimental group consisted of more patients in the inter. nai motivation action phase and fewer patients in the precontemplation phase. The experimental group also included significantly more patients who had a low educational llevel, i.e., up to secondary wocational school, and fewer patients who had previously been admitted for CVD. As regards the cognitive factors, experimental patients reported significantly stronger intentions to quit smoking after hospital discharge, reparted more pros of quitting and perceived more social support. Finaly, the experimental group included more patients who were admitted to a general hospital and whose treatment condition was self-selected by the hospital. Because the latter two factors concerned hospital characteristics, while patients were the units of analysis, type I errors could have occurred due to ignoring the nested design. However, this could not have been the case for other baseline characteristics, because they were measured at patient level. All baseline variables were included as covariates in the regression analysis. 
Tablte 4.1

Baseline characteristics for experimental and control cardiac inpatients $(N=789)$

Daseline characteristes tor experimenta

$\begin{aligned} & \text { Experimental group Control group } \\ & (N=388)\end{aligned} \quad$ P-value
$(N=401)$

Pror admisson for $6 \mathrm{DO}$ \%

$25 \%$

$39 \%$

$<.01$

Educational level, up to secondan wocational school \%

$86 \%$

$78 \%$

$<.05$

Reason for admission. $\%$

Myocardial intarction

Angina Pectors

Unknown

Orheri,

$37 \%$

$23 \%$

$24 \%$

$16 \%$

$<.01^{*}$

Intertion to quit mean $\pm \$ 0$

(1)wery weak, very strong $=10$ )

$7.74 \pm 2.2$

$30 \%$

$25 \%$

$11 \%$

$35 \%$

Motwatinal phase, \%

precontemplation

contemplation

external motivation action phase

internal motiation action phase

$\begin{array}{ll}1 \% & 10 \% \\ 7 \% & 12 \% \\ 29 \% & 35 \% \\ 63 \% & 43 \% \\ 178+05 & 168\end{array}$

Pros, mean \pm SO $10=$ no pros, 3=many pros)

$1.78 \pm 0.5$

$1.68 \pm 0.6$

$<.05$

Cons, mean \pm SD (0wa cons, $3=$ many cons)

$-1.28 \pm 0.7$

$-1.34 \pm 0.7$

N.S.

Soctal support, mean \pm 50

( 3 -mich discouragement, $3=$ much support)

$1.38 \pm 0.9$

$1.04 \pm 1.0$

$<.01$

Madeling, mean \pm SD

(- leeveryone smokes, 1 =no one smokas)

selfelficacy expectations, mean \pm 50

( $3=$ very dillicult, 3 -very easy)

$0.09 \pm 0.4$

$0.06=0.4$

N. 5 .

$-1.55 \pm 1.3$

$-1.66 \pm 1.3$

N.S.

$17.2 \pm 4.5$

$17.1 \pm 4.3$

N.S.

Level of addiction, Fagerstrom, mean st $5 \mathrm{D}$

( $1=$ no physical addiction, $10=$ serious addiction)

$4.3 \pm 2.3$

$45 \%$

$56.2 \pm 10.6$

$65 \%$

$79 \%$

$78 \%$
$4.4 \pm 2.4$ N.S

$55 \%$

N.S

$57.3 \pm 10.9 \quad$ N.S.

$46 \%$

$54 \%$

$78 \%$
$<.01$

Self selection of treatment, \%

Type of hospltal fgeneral hospital). $\%$

Mens, we

foducational level up to lower level wocational traning degree; indudes those patients whose reason for admission was missing inclucies those patients whose reason for admission was coronary angioplasty cardiac dyshythmias or decompensa. tio cordis, "hese reasons for admission were combined because of the rarly of their occurrence, "p-walues concern overall chisquare tess $(d t=3)$; $\$$ < 01 assuming patients as wnits of analysis: for hospitals as unit of analysis table 4.3 shows that there was no treatment irbalance with respect to seff-selection of treatment and type of hospitat.

\section{Effects on Smoking Cessation}

As can be seen in table 4.2, chi-square analysis excluding patients lost to follow-up or regarding such patients as smokers showed significantly higher percentages of point prevalence and continuous abstinence in the experimental group. 


\section{Table 4.2 Smoking cessation after welve months}

without patiens lost to follow-up $(\mathrm{N}-554)$ intention-to-lreat $(\mathrm{N}=76 \mathrm{~s})$

point prewalence abstinience*

63.1

55.8 continumos abstinence *

42.2

33.3 point prewatence abstinamen

439

38.8 continuous abistinence

31.8

25.1

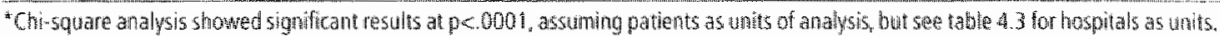

Vewing the percentages of quitting for each individual hospital, table 4.3 shows that the experimental hospitals generally showed higher point prewalence and continuous abstinence cessation rates than the control hospitals, though the differences were substantially smaller with respect to the intention-to-treat analysis. However, one control hospital (hospital 4) showed substantially higher cessation rates than the other control hospitals. Furthermore the mean cessation percentages calculated for the individual experimental and control hospitals (see Table 4.3) were in the analysis excluding patients lost to follow-up like those found in the chi-square analyses, but were slightly smaller for the intention-to-treat analyses (see also Table 4.2). The table is only descriptive, however since its contents are raw percentages unadjusted for baseline differences and without significance test.

\section{Table 4.3 Smoking cessation per hospital (\%)}

\begin{tabular}{|c|c|c|c|c|c|}
\hline & \multirow[t]{2}{*}{ dropout $1 \%$} & \multicolumn{2}{|c|}{ without patients lost to follown up } & \multicolumn{2}{|c|}{ intention-to-treat } \\
\hline & & $\begin{array}{l}\text { point prevalence } \\
\text { abstinerce }\end{array}$ & $\begin{array}{l}\text { continuous } \\
\text { abstinence }\end{array}$ & $\begin{array}{c}\text { point prevalence } \\
\text { abstimence }\end{array}$ & $\begin{array}{l}\text { continuous } \\
\text { abstinence }\end{array}$ \\
\hline \multicolumn{6}{|l|}{ experimental condition: } \\
\hline hospital $1^{\circ}$, is $(\mathrm{N}=80)$ & 29 & 61 & 56 & 44 & 40 \\
\hline hospital $2^{2}(N=162)$ & 29 & 68 & 58 & 48 & 42 \\
\hline hospital $3(N=39)$ & 28 & 64 & 57 & 46 & $4 \|$ \\
\hline hosphtal $4(N=48)$ & 33 & 56 & 47 & 38 & 31 \\
\hline hospital $5(N=45)$ & 38 & 54 & 54 & 33 & 33 \\
\hline \multicolumn{6}{|l|}{ control condition: } \\
\hline hospitat ia $(N=117)$ & 28 & 44 & 36 & 32 & 26 \\
\hline hospital $2^{\circ} \quad(N=62)$ & 24 & 28 & 23 & 21 & 18 \\
\hline hospital $3^{\circ} \quad(N=62)$ & 26 & 35 & 22 & 26 & 16 \\
\hline hospital $4(N=78)$ & 19 & 60 & 51 & 49 & 41 \\
\hline hospital $5(N=64)$ & 27 & 36 & 26 & 27 & 19 \\
\hline mospitial $\overrightarrow{6}(N=7)$ & 0 & 43 & 43 & 4 & 43 \\
\hline \multicolumn{6}{|l|}{ mean $\%$ the } \\
\hline experimental hospitals & 31 & 61 & 54 & 42 & 38 \\
\hline mean $\%$ six control & 21 & 41 & 33 & 33 & 27 \\
\hline
\end{tabular}

scaf-selected hospital; university hospital. 
Logistic regression of both outcomes with both methods (including and exduding patients lost to follow-up) revealed no interaction between treatment and reason for admission, motivational phase or self-selection of treatment (all $p>.40$ using likelihood ratio tests).

The logistic regression analysis for point prevalence abstinence excluding patients lost to follow-up (Table 4.4) showed a significant intervention effect $(O R=1.63,90 \%$ $\mathrm{Cl}=1.13-2.33, \mathrm{p}=.02)$, but this result was not confirmed by the intention-to-treat analysis $1 O R=0.97,90 \% \mathrm{Cl}=0.71-1.34, \mathrm{p}=.45 \mathrm{j}$. Furthermore, as table 4.4 shows, neither of the two analyses showed a main effect of the indicator for self-selection of treatment on the outcome (all $p>.45$ ).

The intervention effect on continuous abstinemce (Table 4.5) was also significant only when patients lost to follow-up were excluded lanalysis excluding patients lost to follow-up: $O R=1.86,90 \% C I=1.30-2.66, p=.003$; intention-to-treat: $O R=1.17,90 \%$ $\mathrm{Cl}=0.85-1.61, p=.43)$. As shown in table 4.5, the main effect of the indicator of selfselection was not significant either $(p>43$, irrespective of whether patients lost to follow-up were included).

Re-analysis of the regression models in tables 4.4 and 4.5 with random effect logistic regression in EGRET (Statistics and Epidemiology Research Corporation, 1993) revealed treatment effects similar to those found with SPSS. The EGRET analysis on point prevalence abstinence showed an odds ratio of $1.63(\mathrm{p}=.03)$ for treatment excluding patients lost to follow-up, and an odds ratio of $0.98(p=92)$ for treatment in the intention-to-treat analysis. EGRET analyses on continuous abstinence showed an odds ratio of $1.86(p=.005)$ for treatment excluding patients lost to follow-up and an odds ratio of $1.17(p=22)$ for treatment in the intention-to-treat analysis. The random hospital effect was not significant in any of the four analyses (all $p>.40$ ).

The absence of interactions between (1) treatment and motivational phase, and (2) treatment and reason for admission were contrary to our expectations. The absence of an interaction between treatment and motivational phase could have been caused by a lack of power, since the variable consisted of four categories, which necessitated the use of df(3) to test the interaction, and had a skew distribution with the first two categories underrepresented (see also Table 4.1). To check whether the absence of significant interaction was due to a lack of power, we re-analyzed the final regression models, including the interaction between phase of change and treatment with phase of change dichotomized. Based on our expectations that internally motivated patients would profit significantly more from the intervention than patients in the other motivational phases, precontemplators, contemplators and externally motivated actors were categorized as one group (reference group, $\mathrm{N}=351$ ) and internally motivated actors. $\langle N=391)$ as the comparison group. These analyses showed no interaction either ( $p>$ s0). Dichotomization of reason for admission was deemed unnecessary because the sample was equally distributed among the various reasons for admission and all $p>.40$ for the interaction between reason and treatment. 


\section{Table 4.4 Predictors of point prevalence} abstinence

\section{Variable}

B SE Odds Ratio $P$

Experimental (1) versus control 10 )

$0.48 \quad 0.22$

1.63

$02^{\text {五 }}$

B

SE Odds matio

Prior admission (1) versus

no prior admission for CVD (0)

$\begin{array}{llll}-0.29 & 0.24 & 0.75 & 24\end{array}$

$-0.03$

0.19

0.97

.45

Up to secondary vocational school (1)

versus higher education 10

$\begin{array}{llll}-0.34 & 0.26 & 0.71 & .20\end{array}$

$-0.42$

$0.21 \quad 0.66$

.05

Reason for admission abc

Admissian for Myocardial

Infarction (1) versus other (0)

Admission for Angina

Pectoris (1) versus other (0)

Admission for unknown (1)

versus other $10 \mathrm{p}$

Pretest intention to quil

0.54

0.27

$1.71 \quad .05$

0.54

$0.24 \quad 1.71$

.03

$\begin{array}{llll}0.52 & 0.29 & 1.68 & 08\end{array}$

0.51

$0.26 \quad 1.66$

.05

$\begin{array}{llll}0.16 & 0.33 & 1.17 & .63\end{array}$

0.18

0.28

0.53

$0.11 \quad 0.05$

$11.12 \quad .03$

0.16

0.04

1.18

.001

Motivationall phase thes

Contemplation phase (1)

versus precomtemplation

phase (0)

$\begin{array}{llll}-0.49 & 0.69 & 0.61 & .48\end{array}$

$-0.38$

0.63

0.68

.54

Externa! motivation

phase (1) versus

precontemplation phase $(0)$

Internal motivation phase (1)

0.39

0.58

1.47

.51

0.41

0.53

1. 50

.44

versus precontemplation

phase (0)

0.63

0.61

189

.30

0.71

0.54

2.04

.19

Mean pretest score on pros $(0 /+3)$

$0.18 \quad 0.20$

$1.19 \quad .39$

$-0.04$

0.17

0.96

.83

Mean pretest score an cons $(0 /-3)$

$-0.04 \quad 0.17$

$0.96 \quad .82$

$-0.001$

0.14

0.99

.99

Mean pretest score

or sociall suppoit $(3 /+3)$

$\begin{array}{llll}0.19 & 0.12 & 1.21 & .11\end{array}$

0.14

0.10

1.15

.16

Mean pretest score on modeling $(-1 /+1) 0.59$

0.27

$1.81 \quad .03$

0.83

0.23

2.28

.009

Mean pretest score on self-efficacy

expectations $(-3 /+3)$

$\begin{array}{llll}0.24 & 0.10 & 1.27 & .01\end{array}$

$0.11 \quad 0.08$

1.12

.14

Age at smoking initiation

$\begin{array}{llll}0.03 & 0.03 & 1.03 & 31\end{array}$

0.01

0.02

1.01

.65

Level of addiction, FTND score (1-10)

$-0.07$

0.05

$0.94 \quad .17$

$-0.09$

0.04

0,91

.03

Previous quit attempt. yes $(1)$, no $(0)$

$\begin{array}{rr}-0.36 & 0.23\end{array}$

$0.70 \quad .12$

$-0.31$

0.19

0.73

.11

Ange (28/86)

$0.02 \quad 0.01$

$1.02 \quad .10$

0.01

0.01

1.01

.45

Sel-selection of treatment.

yes (1), no (0)

$\begin{array}{llll}0.13 & 0.23 & 1.94 & 56\end{array}$

0.14

0.20

1.16

46

Type of hospital, general (1),

uniwersity (0)

$\begin{array}{llll}0.004 & 0.24 & 1.00 & .99\end{array}$

0.03

0.21

1.03

.87

Gender, woman (1) versus man (0)

$-0.17 \quad 0.25$

$0.84 \quad .49$

$-0.29$

0.21

0.75

.17

Constant (intercept)

$-2.68 \quad 0.98$

$-2.38$

0.8

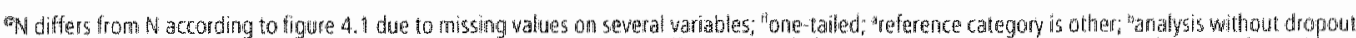

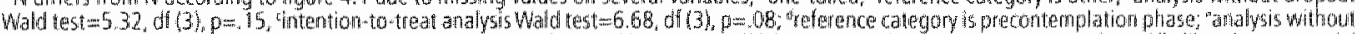

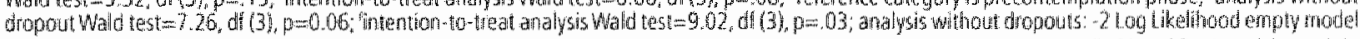

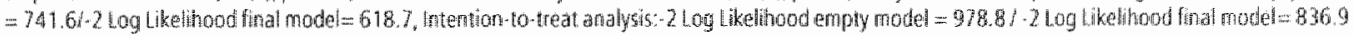




\section{Table 4.5 Predictors of \\ continued abstinence}

without dropouts $\left(N=536^{\circ}\right)$

Variable B SE Odds Ratio $P$

Experhmental 11 wersus control 10 .

0.62

0.22

1.86

$.003^{33}$

$\begin{array}{llll}-0.50 & 0.25 & 0.61 & .05\end{array}$

$-0.57 \quad 0.27$

0.57

.03

0.28

$1.95 \quad .02$

$1.48 \quad .19$

$0.39 \quad 0.30$

$1.04 \quad .90$

0.040 .33

$0.07 \quad 0.05$

$1.09 \quad .21$

Pretest intention to quit

Motivational phaseded
Contemplation phase (1)

wersus precontemplation

phase (o)

External motwation phase (1)

wersus precontemplation

phase (0)

Internal motivation phase (1)

versus precontemplation phase (o)

Maan pretest score on pros $(0 /+3)$

Mean pretest score on cons $(0 /-3)$

$-0.75 \quad 0.77$

0.47

.33

$-0.70$

0.71

0.50

33

$\begin{array}{llll}0.48 & 0.62 & 1.62 & .44\end{array}$

0.46

0.58

9.58

Meari pretest score

on social support $(-3 x+3)$

$0.66 \quad 0.64$

$1.93 \quad .31$

0.110 .20

$1.11 \quad .59$

$\begin{array}{rr}-0.28 & 0.17\end{array}$

$0.76 \quad .10$

$0.16 \quad 0.11$

$1.18 \quad .15$

$1.69 \quad .05$

0.27

$1.27 \quad .01$

$1.04 \quad .14$

$0.97 \quad 60$

$0.78 \quad .28$

Previous auir attempt, yes (1), no (0)

Age 28186)

$0.24 \quad 0.09$

$0.04 \quad 0.02$

0.05

0.23

$1.00 \quad .96$

Selt-selection of veatment.

yess (1), no (0)

Type of hospital, general $(1)$.

uniwersity (0)

$0.01 \quad 0.01$

$1.12 \quad .62$

$0.11 \quad 0.23$

$0.97 \quad .89$

$0.68 \quad .14$

01

$\begin{array}{rrrr}0.70 & 0.59 & 2.01 & .24 \\ -0.07 & 0.18 & 0.93 & .70 \\ -0.18 & 0.15 & 0.84 & .22\end{array}$

$\begin{array}{llll}0.13 & 0.10 & 1.14 & .20\end{array}$

$\begin{array}{llll}0.79 & 0.24 & 2.20 & .001\end{array}$

Gender woman (1) wersus man 10$) \quad 0.39 \quad 0.26$

$-2.44 \quad 0.98$

0.14

0.08

$1.15 \quad .07$

$0.02 \quad 0.02$

$1.02 \quad .4$

$-0.05$

0.04

$0.95 \quad .24$

$0.23 \quad 0.20$

$0.79 \quad .25$

$\begin{array}{llll}-0.01 & 0.01 & 0.99 & .52\end{array}$

Constant (intercept)

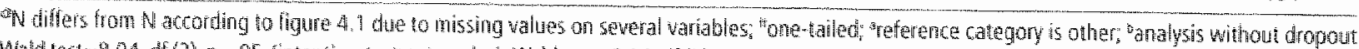

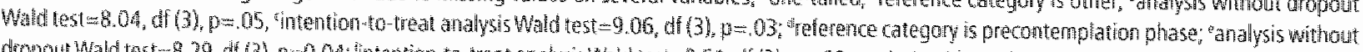

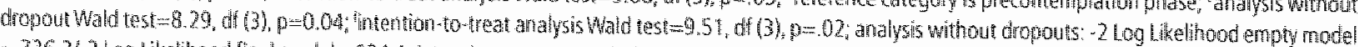

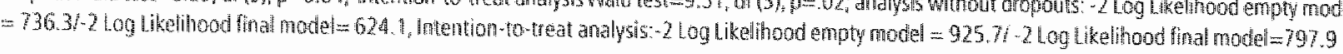




\section{Predictors of Quitting}

Tables 4.4 and 4.5 show which baseline characteristics were significantly associated with patients'smoking status at follow-up. Table 4.4 shows that point prevalence abstinence was positively associated with patients intention to quit smoking and with the perception of more non-smokers in the social environment (modeling), regardless of whether patients lost to follow-up were categorized as smokers. However, inconsistent results were found for baseline self-efficacy expectations and prior admission for CVD. Not having been admitted before for CVD only proved to be associated with point prevalence abstinence in the intention-to-treat analysis, while higher self-efficacy expectations were only significantly associated with this outcome in the analysis excluding patients lost to follow-up.

Slightly different predictors were found for continuous abstinence (Table 4.5). Modeling was the only predictive factor for continued abstinence that was also found for point prevalence abstinence, regardless of the type of analysis. In contrast to the predictors of point prevalence abstinence, prior admission for CVD, level of education and reason for admission were also significant predictors of continued abstinence, regardless of the type of analysis. Although some more predictive variables were found for continued abstinence, they were not consistently reported in the analyses excluding patients lost to follow-up or regarding them as smokers. As can be seen in table 4.5, pretest scores on self-efficacy expectations were only predictive of the outcome when patients lost to follow-up were excluded from the analysis, while intention to quit and gender were only predictive variables in the intention-to-treat analysis. Furthermore, subanalyses with phase of change dichotomized (not presented in tables 4.4 and 4.5 , but see previous subsection for details) showed this factor to be significantly associated with both outcomes in the analyses excluding patients lost to follow-up.

A check of assumptions of logistic regression gave satisfactory results: no independent variables showed collinearity, and none of the patients could be considered an outlier or influential case.

\section{Discussion}

The present study examined the one-year effectiveness of C-MIS on smoking ces. sation in two ways. In the first approach, patients lost to follow-up (27\%) were onitted from the analysis, while the second involved an intention-to-treat procedure. The latter procedure has been recommended by various researchers as a protection against biased treatment effects due to patient selection (Dignan, 1993; Fiore el al., 2000; Rice \& Stead, 1999: Rossi \& Freeman, 1993). On the other hand, treating all patients lost to follow-up as posttest smokers may yield a too consenvative estimate of the effect if 
there is substantial dropout, as in the present study (Dignan, 1993; Little \& Yau, 1998; Rossi \& Freeman, 1993). The intention-to-treat analysis should therefore not be regarded as providing the ultimate truth.

The results showed significant intervention effects on both outcomes when patients lost to follow-up were not included, but the effects were no longer significant when they were regarded as smokers. No evidence was found that the differences in the randomization procedure, resulting in self-selection by four of the eleven hospitals, had influenced the treatment effect (as an interaction effect) or the smoking cessation outcomes (as a main effect). But even if there was a main effect of self-selection on the smoking outcomes, this was adjusted for by retaining the indicator for self-selection in the regression models. This adjustment procedure was also carried out for the baseline differences found and for other possible covariates. Furthermore, the random effect logistic regression analyses failed to show a random hospital effect and confirmed the SPSS analyses in terms of the size and lack of significance of the treatment effects. since no interaction was found between the treatment condition and either the reason of admission or the motivational phase of change, there is no evidence that the treatment effect depended on the reason of admission or the motivational phase.

In sum, it seems that the outcomes have not been distorted by the absence of complete randomization or the presence of certain baseline differences between the two conditions. In view of the substantial loss to follow-up rate and its relation to condition and some baseline characteristics, it seems wise to give more weight to the intention-to-treat analyses. Moreover, most other studies among cardiac inpatients have used intention-to-treat results to judge effectiveness (Johnson et al., 1999; Ockene et all. 1992; Rigotti et al., 1994; Taylor et al., 1990). Hence, it must be conduded that, in contrast to the results after three months of follow-up (Bolman et al., in press), the present study failed to show effectiveness at twelve months after the intervention.

Comparison of our results with those of other studies among cardiac impatients is hindered by differences in the methodologies used. Three of the five studies reported in the literature included biochemical walidation (Ockene et al., 1992; Rigotti et al., 1994; Taylor et al., 1990), while four used intention-to-treat procedures (Johnson et al., 1999; Ockene et al., 1992; Rigotti et al., 1994; Taylor et al., 1990). Furthermore, all interventions were more intensive than ours, while two of the five studies measured the effects at six and eighteen months (Johnson et al., 1999; Rosal et al., 1998), and most studies used a truly randomized controlled trial (Ockene et al., 1992; Rigotti et al., 1994; Rosal et al., 1998; Taylor et al., 1990). Finally, our loss to follow-up rate was higher, which may have led to more conservative estimates in our study on the basis of intention-to-treat analysis.

As in the present study, two other studies also failed to find an effect (Johnison et al., 1999; Rigotti et al., 1994), Johnson and colleagues (1999) measured six-months (intention-to-treat) effects by self-reports in a quasi-experimental study. They attributed the absence of a significant effect to a type 11 error ( $46 \%$ smoking abstinence in the 
intervention group, $31 \%$ in the control group). However, their loss to follow-tup rate was only $11 \%$, compared to $27 \%$ in our study. In their randomized controlled tral, Rigotti and colleagues (1994) found identical twelve-months continuous non-smoking rates in the intervention and control groups $(51 \%)$ and a small, non-significant difference in point prevalence abstinence (61\% in the intervention graup. $54 \%$ in the control group). They reported no loss to follow-up, attributed the absence of effects to low strength of the intervention and bad timing, and suggested targeting patients after discharge from hospital. In contrast to our findings Taylor and colleagues (1990) found a significant intervention effect in their biochemically validated intention-to-treat analysis of point prevalence abstinence $(61 \%$ versus $32 \%$ in the intervention and control groups, respectively). Their intervention was more intensive and used a long period of aftercare. Ockene and colleagues (1992) also reported an intervention effect, but only for patients with severe myocardial infarction. This effect disappeared at the eighteenmonths follow-up measurement (Rosal et al., 1998). Miller and colleagues (1997) showed a significant intervention effect on (intention-to-treat) point prevalence abstinence $(O R=1.6$ compared to usual care). Their repeated telephone support after discharge (four calls) increased the cessation rate significantly after one year. Two meta-analyses showed that high cessation rates can be achieved if health care providers collaborate and use behavioral counseling, micotine replacement therapy and aftercare (Fiore, Jorenby, \& Baker, 1997; Rice \& Stead, 1999).

In conclusion, comparison of our study with the successful sudies described above revealed that our effect estimations were more conservative, so the effect may have been underestimated. The studies also suggest, however, that our intervention could be improved by including intensive aftercare with several follow-up telephone calls and nicotine replacement therapy, while the in hospital counseling might also need to become more intensive. Furthermore, process evaluation results obtained at our three months follow-up and described elsewhere (Bolman et al. in press) also indicate that the implementation of the interwention needs improvement, since the present results were obtained under conditions of moderate intervention coverage (i.e., about onefifth of the experimental patients indicated that they had not been exposed to one or more of the components of the intervention).

Both point prevalence and continuous abstinence were predicted by the perception of fewer smokers in the environment, while a strong pretest intention to quit and not having been previously admitted for CVD were onlly significant predictors in the intention-to-treat analyses on both outcomes. The significance of other factors differed slightly between the outcomes and methods. Application of a Bonferroni type adjustment for intlated type I errors (i.e., $p<.003 ; p=05 / 17$ covariates) may imply that intention-to-treat point prevalence abstinence as well as continuous abstinence were only related to the number of smokers perceived in the environment (modeling) and the intention to quit at the pretest, while no significant predictors were found when patients lost to follow-up were left out of the analyses. Most factors identified in the 
present study were also found in other studies among CHD patients (Greenwood et al., 1995; Mckenina Higgens; 1997; Ockene et al., 1992; Orleans et al. 1990; Rice et al., 1996; Rigotti et al., 1994; Rosal et al., 1998; Taylor et al., 1990; Taylor et al., 1996; Van Berkel et al., 2000; Van Berkel et al., submitted-b), although we unable to replicate the influences of age that was reported in a review paper (Mckenna \& Higgens, 1997). Mast studies that predicted on the predictors of smoking cessation among CHD patients, however, clid not correct for multiple testing. which impedes comparison.

The present study was subject to several weaknesses. First the randomization procedure failed for four hospitals. However our results showed similar treatment effects for patients in the self-selected hospitals and those in the randomized hospitals. Furthermore, although no main effect of self-selection was found either, we corrected for its possible existence by retaining this factor in the regression models. Hence, it is unlikely that the results were distorted by the failure of randomization. Second, loss to follow-up rates were high despite three postal reminders, a telephone reminder and the provision of an incentive by making patients eligible to win a $\$ 25$ prize. Many patients had changed their addresses without informing us. It is therefore recommended that future studies use a tracking system based on hospital records (which was not done in the present study). Furthermore, follow-up assessments by telephone are recommended, since most studies among cardiac inpatients that used this procedure reported attrition rates of 10\% or less (Miller et al. 1997; Ockene et al. 1992: Rigotti et al. 1994; Taylor et al., 1996). Third, selective loss to follow-up occurred on variables that are known to be predictors of smoking abstinence among CHD patients (Johnson et al., 1999; Ockene et al., 1992; Rigotti et al., 1994). Since the effects found in the analysis excluding patients lost to follow-up may therefore have been overestimated, we based our conclusion concerning the effectiveness of the intervention on the intention-to-treat analyses. It might, however, have affected the external validity of the study. Fourth, no biochemical confirmation was used. Efforts were undertaken to overcome this deficiency by using an identification coding system, by assuring patients" anonymity and confidentiality, and by applying a bogus pipeline procedure, which has been justified in a number of studies (Hansen et al., 1985; Murray et al., 1993; Velicer et al.. 1992). However, while several interwention studies in the general population (Glasgow et al. 1993; Kottke et al., 1988; Murray et al., 1993) as well as for CHD patients (Miller et al., 1997: Ockene et al., 1992; Taylor et al., 1990; Van Berkel et al., submitted-b) have reported that treatment groups are not more likely to misrepresent their smoking behavior than controls, patients may have under reported or falsely reported their smoking behavior. Therefore it is unsure whether the abstinence rates we found in both treatment groups reflect real abstinence rates, and this might affect the generalizability of the results. Fifth, nurses in the experimental group could not be blinded for treatment condition. Although the recruitment procedure was supervised by internal program co-ordinators and nurses were explicitly instructed to ask every eligible patient, nurses may have been selective in their recruitment (selection bias). Therefore, 
the experimental group in particular may not represent the average population of CHD inpatients. Sixth, despite our request, neither the numbers of all smoking patients admitted to the hospital, nor nurses' adherence to the intervention were monitored by the internal program co-ordinators. This might be due to the fact that ward nurses rather than research nurses were responsible as is common in the Netherlands. There. fore, it is unclear whether the sample of smoking cardiac inpatients was representati. we. The long period needed to include sufficient numbers of patients suggests that not all smoking patients, were approached. Nurses confirmed this, mentioning time constraints and forgetfulness as the most important causes. Seventh, few follow-up contacts by telephone occurred. Initially, nurses were supposed to call patients once after discharge from hospital. This was ultimately refused by ward managers due to time constraints. Because high-quality aftercare is essential (Lando, Hellerstedt, Pirie \& McGovern, 1992; Lichtenstein, Glasgow, Lando, Ossip-Klein, \& Boles, 1996; Miller et al., 1997; Taylor et al., 1996; Zhu et al., 1996), efforts should be made to make telephone follow-up feasible. An alternative to phone calls may be computer-generated personalized letters (De Vries \& Brug, 1999; Dijkstra et al., 1998a; Velicer, Prochaska, Bellis, Diclemente, \& et al., 1993). Finally, our study did not include a cost-effectiveness assessment. Reviews on the treatment of hospitalized smokers suggest , however $_{i}$ that brief treatments, including interpersonal counseling and self-help, are cost-effective (Orleans et al., 1993b; Raw et al., 1998; U.S. DHHS, 1996).

Despite these limitations, the study provided important insight into the effectiveness of the intervention in real clinical circumstances. It suggests that the intevention is eligible for improvement in order to achieve longterm effects. The study furthermore contributed to the theoretical insights into the factors associated with smoking cessation in CHD patients, which is also relevant for clinical practice. 


\section{Chapter five}

\section{FACTORS DETERMINING CARDIAC NURSES" INTENTIONS TO CONTINUE USING A SMOKING CESSATION PROTOCOL*}

\section{Abstract}

Objective: To facilitate the continued use of a smoking cessation protocol among cardiac nurses by assessing their intentions and motives for continuation. A model combining Attitude, Social influence and Self-efficacy constructs as predictors of intentions (ASE model) served as the theoretical framework.

Setting: Cardiology wards in 5 hospitals.

Respondents: Eighty-five nurses working in the cardiology wards of these hospitals.

Outcome measures: Nurses' intentions and motives for continuing to use a smoking cessation protocol for smoking patients.

Results. Greater perceived simplicity and advantages of the protocol were associated with increased intentions to continue, while perceived social influences and selfefficacy were not. The influence of the level of experience in using the protocol and the nurses' own smoking behavior was mediated by attitude about the smoking cessation protocol.

Conclusions. Nurses not intending to continue using the protocol need to be convinced of the advantages of working with such a protocol, and of its user-friendliness (simplicity).

"Accepted for publication as: Bolman, $\mathrm{C}_{\text {, }}$ De Vries, H., Mesters, I. Factors detêrmining cardiac nurses" intentions to continue using a smoking cessation protocol. Heart and Lung: The journal of acute and critical care. 


\section{Introduction}

Admission to a cardialogy ward is often regarded as an excellent opportunity for health professionals to help patients to quit smoking. During hospitalization, patients are more aware of their personal vulnerability to the dangers of smoking and are more motivated to quit (Emmons \& Goldstein, 1992; Orleans et al s, 1990; Taylor et al., 1990). 5 moking cessation is relevant for patients admitted with coronary heart disease (CHD) since it improves the prognosis more than any other treatment (Deckers et al., 1994; Mehta \& Eagle, 1998; Smith, 1997; U.S. DHHS, 1990\%.

Cardiac nurses have the greatest frequency and duration of contact with patients, are a continuous factor in hospital care, and are recognized patient educators (DeMello et al., 1989; Nagle et al., 1999). Hence, they are considered appropriate intermediaries in assisting cardiac inpatients to quit smoking. For this reason, a smoking cessation protocol, with which Dutch cardiac nurses could assist their patients to quit smoking, was developed and tested for effectiveness. The protocol included the four A's (Ask, Advise, Assist, Arrange), as recommended in the "Clinical Practice Guideline for treating tobacco use and dependence" (Fiore et al, 2000).

The impact of such interventions is, however, not only determined by their effectiveness, but also by the level of continued use after the trial period (De Vries, 1998; Oidenburg, Sallis, French, \& Owen, 1999). According to Rogers (1995), continuation (also often referred to as maintenance or institutionalization), is the final phase in the process of diffusing an innovative program. The likelihood of continued use of an intervention can be increased by improving our understanding of the factors determining continued use (Bartholomew et al., 1998; Oldenburg, Hardcastle, \& Kok, 1997; Oldenburg et al., 1999; Rogers, 1995; Van Assema, Brug, Glanz, Dolders, \& Mudde, 1998).

The present study assessed nurses' intentions and underlying motives for continuing or discontinuing the use of the smoking cessation protocol. While some descriptive studies have analyzed nurses' motives for helping patients to quit smoking (Faullkner \& Ward, 1983; Goldstein et al., 1987; Nagle et al., 1999; NiPO, 1995; Wiebing \& Baari, 1997), no study has yet been conducted to analyze inurses' intentions and motives for providing protocol-based smoking cessation assistance in relation to the process of continuation.

For the purpose of the present study, the Attitude, Social influence and Self-efficacy model or ASE model (Figure 5.1) was used as a theoretical framework (De Vries \& Backbier, 1994; De Vries et al., 1988: De Vries \& Mudde, 1998). The ASE model has been used to explain and predict various health education behaviors of individuals and intermediaries like teachers (Dijkstra, 1995; Paulussen, Kok, \& Schaalma, 1994). The ASE model can be regarded as an extended version of the Theory of Planned Behavior (TPB) (Ajzen, 1991), which has been used as a theoretical framework in explaining nurses' intentions toward providing homecare and assessing patients' pain (Nash, Edwards, \& Nebauer, 1993: Vermette \& Godin, 1996). Like the TPB, the ASE model combines the constructs of 
Atticude, Social influence and Self-efficacy expectations (which is a concept comparable to behavioral control) as predictors of intentions (De Vres \& Backbier, 1994: De: Vies et al. 1988; De Wries \& Mudde, 1998; Kok at al, 1991). In addition, extemal variables such as demographic factors are assumed to influence behavioral intention through these determinants. The ASE madel, however, measures the concept of social influence more extensively (De Vries et al., 1995; De Vries \& Mudde 1998). The ASE model as used in the present study includes the concepts of social support and modeling as social predictors of intention. Compared to the TPB, the ASE model has evolved as a separate model with several differences, predominantly of a methodological natu. re (De Vries \& Mudde, 1998).

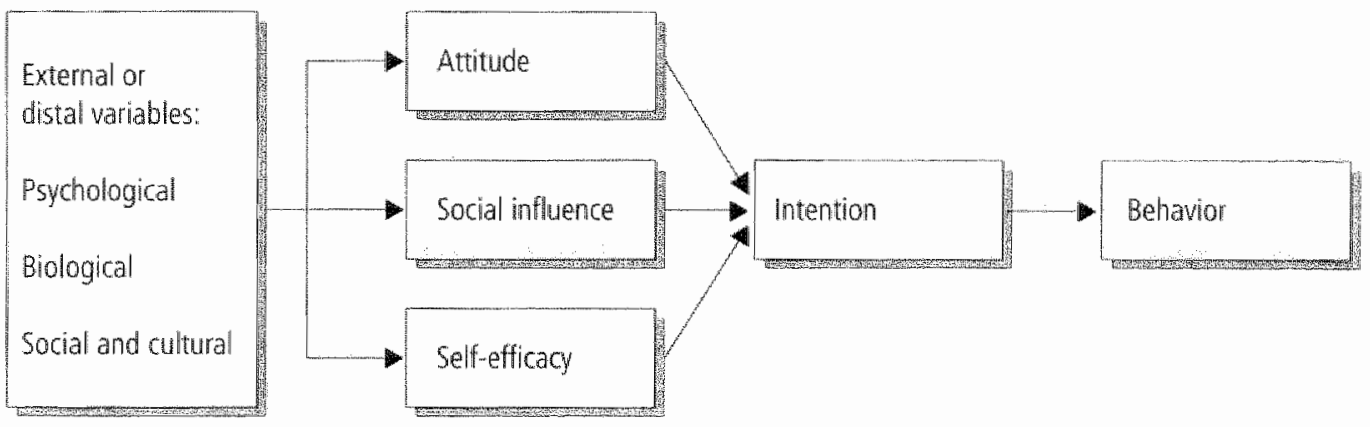

Figure 5.1. ASE model (adapted from De Vries \& Mudde, 1998)

The ASE model as applied in the present study suggests that nurses intentions to continue using the protocol are determined by the three psycho-social factors mentioned above. Whether nurses have a positive attitude toward the continued use of the protocal depends on the extent to which they perceive advantages and disadvantages of its use. Vartous studies have suggested that the perceived superionity of a new health education practice over the existing practice and the perceived complexity of the new practice are important in this respect (Dijkstra, 1995; Mesters \& Meettens, 1999; Rogers, 1995; Stekker, Goodman, Mcleroy, Davis, \& Koch, 1992). In terms of the A5E model, perceived superiority of the protocol can be seen as an advantage to use it: while perceved complexity can be seen as a disadvantage to use it. With regard to the social influences, the support experienced from important others in the work environment (e.g. from superwisory staff), and the behavior perceived in the work environment (e.g., in colleagues), are expected to play a role (De Vries et al., 1995; Duncan et al. 1991; Lechner, 1998; Lllington, 1997). With respect to self-efficacy expectations, it is expected that nurses who perceive themselves to be able to apply the protocol apmpropriately will be more likely to have the intention to continue using the protocol. 
Because the present study focused on the explanation of nurses intentions to continue using the protocol, and nurses had already gained some experience in wor. king with the protocol during an effectiveness study, the level of experience in using the protocol was added to the theoretical model. Several researchers have suggested that continued use may be more likely to occur after a certain degree of routine has been established in daly practice (Goodman \& 5teckler, 1989; Steckler \& Goodman, 1989; Yin, 1979). The ASE model assumes that prior experience with the behavior in question (which relates to level of experience) will impact on the intentions indirectly by influencing attitudes, social influence perceptions and self-efficacy expectations (De Vries \& Mudde, 1998). It was hoped that the present study might provide further insight into the role of the level of experience in explaining nurses' intentions.

In summary, the first goal of the present study was to analyze psycho-social differences between nurses who intend to continue using the protocol and those who do not intend to continue using it. The second goal was to describe the applicability of the ASE model by assessing whether nurses. intentions can be explained by the model.

\section{Methods}

\section{Respondents}

The present study involved nurses ( $N=164$ ) who worked in cardiology wards in the five hospitals that participated as the experimental group in a smoking cessation effectiveness study. The present cross-sectional survey took place one week after the effectiveness study had been concluded. All nurses working in the ward at the time of the survey were eligible for enrollment, except those doing internships and those in training to obtain their nursing degree. Within these criteria, nurses levels of experience in working with the smoking cessation protocol could vary, because nurses who had started to work in the ward at any time during the effectiveness study could participate.

\section{Smoking Cessation Intervention}

The smoking cessation intervention was labeled the Minimal Intervention Strategy for Cardiac inpatients (C-MIS). Its core elements consisted of six steps. First, each patient was briefly advised by their cardiologist to quit smoking. Second, ward nurses made a brief assessment of the patient's smoking behavior, degree of addiction, and motivation to quit (the so-called smoker"s profile). In step 3; nurses discussed the advantages and disadvantages a patient perceived with regard to smoking cessation, in 
order to motivate unmotivated patients to quit smoking. If patients were alleady motivated to quit, the nurse could omit this step and proceed to step 4 , which included discussing expected probiems, barriers, high-risk situations, and self-efficacy expectations concerning smoking cessation. In this step, nurses provided assistance on how to cope with high-risk situations. If the patient was motivated to quit and prepared to overcome high-risk situations and barriers, the nurse proceeded to step 5 , encoura. ging the patient to set a date for quitting. During hospitalization, nurses also provided the patient with printed supportive materials. Nurses were asked to record the subjects discussed with each patient on an intervention card developed especially for this study. This intervention card was added to the patients' hospital chant in order to inform the cardiologist (who performed the aftercare) about the irhospital part of the intervention. Step 6, which was carried out 4-6 weeks after hospital discharge, occurred when the patient visited the cardiology outpatient clinic for a check-up. At this time, the cardiologist addressed the patient's smoking behavior again, to reinforce quitting behavior in order to prevent relapse, and to motivate patients who had already relapsed to make another attempt to quit.

Before the intervention started, nurses received a standard two hour training from the researcher and colleagues. The training included information on the ationale for and the theory behind the intervention. The steps of the smoking cessation protocol were explained and rehearsed in role-plays. Each ward also received a training manual with instructions on protocol usage and a poster with a description of the protocol. Each nurse received a pocket-size reminder chart including an overview of the protocol and standard messages on the advantages of quitting smoking and how to cope with high-risk situations.

\section{Questionnaire}

The questiannaire was partly based on existing questionnaires or relevant subscales. Information derived from interviews with nurses (unpublished) and from previous research was added when needed or when no existing questionnaire or subscale was available. Where applicable, the approach that was used is addressed in the description of the scales below. The questionnaire was reviewed several times by two experts in smoking cessation during its development.

In agreement with the ASE model, the nurses" intention to continue using the smoking cessation protocol was assessed by the question: "Do you intend to use the protocol in future if it becomes available for free? (5-point answering scale, range: $2=y e s$, definitely; $-2=$ no, definitely not).

Attitudes toward continued use were assessed by means of wo subscalles that, according to Rogers Diffusion theory (1995), reflect important attitudinal altributes of 
a person's decision to adopt a new working method, namely its perceved advantages and perceived complexity. The subscales were derived from a validated quiestionnaire developed by steckler and colleagues ladvantages subscale of four items, complexity subscale of three items), based on Rogers findings (Steckler et al. 1992). This questionnaire was also used in a sudy comparable to the present study, held among Dutch community nurses (Mesters \& Meertens, 1999). The subscales were supplemented with items derived from interviews with nurses and a literature search (Clark, Haverty, \& Kendall, 1990; Faulkner \& Ward, 1983; Goldstein et all, 1987; Latter. Clark, WilsonBarnett, Maben, 1992; Orlandi, 1987; Sanders et al., 1986). The 13-items advantages subscale captured beliefs on general advantages of the smoking cessation protocol (e.g. "In general, the protocol has more advantages than disadvantages"), relative advantages compared to standard care (e.g., "In general, the protocol is an improvement compared to the nomal smoking cessation approach") and beliefs on the effectiweness of the protocol (e.g." "In general, the protocol is a useful tool to prevent relapse after discharge"). A 5-point Likert answering scale, ranging from strongly disagree $(-2)$ to strongly agree $(2)$, was used for each item and an average score for the instrument was calculated. Internal consistency was high (Cronbach's $\alpha=.87$ ). The 3 items complexity subscale measured to what extent the protocol was perceived as complex (Cronbach's $\alpha=66$ ). The wording of the items in this subscale, however, avoided the term 'complexity', asking instead whether, for instance, the respondents found the protocol "easy to understand". In the remainder of this paper, we therefore use the term 'simplicity subscale'. A 5-point Likert answering scale was used for each item, with scores ranging from strongly disagree (-2) to strongly agree (2). The simplicity subscale was constructed in the same way as the advantages subscale, with scores on a 5 -point scale ranging from -2 (indicating that the respondent strongly disagreed with the statements on the simplicity of the protocol) to +2 (indicating that the respondent strongly agreed with these statements).

Two types of social influence were measured, namely support and modeling. Since both constructs were designed to measure the perceived opinions and behavior of several persons together and the correlations between the items may therefore be low, thay were regarded as indexes instead of scales. Hence, no cronbach's alpha coefficients were computed. Both indexes were constructed by adding the answers on the individual items. First, the support murses perceived from colleagues, supervisory stalf and cardiologists was measured on a 5-point scale answering the question "Do you perceive support or discouragement from ......?" (scale ranging from 2 , very much discouragement to +2 , very much support). The social support index measured the extent to which nurses encountered support for providing smoking cessation guidance from the persons mentioned. This index ranged from -6 to +6 , with a negative score indicating discouragement and a positive score indicating support. Second, the perceived level of smoking cessation assistance as modeled by colleagues, supervisory staff and cardiologists (modeling) was measured on a 5-point scale answering the question 
"In your opinion, how many ..... provide smoking cessation assistance?" (scale ranging from 0 , none of the ... provide it, to +4 , all of the ... provide it. The modeling index measured the perceived smoking cessation guidance from the persons mentioned. The sum of the three items ranged from 0 to 12 , with a higher score indicating more persons seen to provide smoking cessation guidance. The persons included in both social influence indexes were chosen on the basis of previously conducted interviews with nurses.

Self-efficacy expectations were measured in accordance with the ASE model by asking nurses how difficult or easy they found it to use the smoking cessation protocol. One question measured the overall self-efficacy toward using the protocol (scale ranging from very difficult $(-2)$ to very easy $(+2)$ ). In addition, self-efficacy expectations toward applying the tasks in the protocol as defined for nurses were measured for each task separately using the same question and answer format. Slightly different from the steps described in the subsection Intervention, the following nurse tasks were decided to be useful to include in the measurement of self-efficacy expectations: (1) draw up a smoker's profile, (2) assess the patient's motivation to quit, (3) address the pros and cons of quitting, (4) heip to overcome barriers to quit smoking. (5) set a date for quit. ting, (6) record subjects discussed in the bedside consultation on the intervention card. Subsequently, a self-efficacy scale was constructed, in which the answers on the overall item and the nurse-specific task items were averaged (Cronbach's $\alpha=.79$ ).

The level of experience in using the smoking cessation protocol (an external varia. ble in the ASE model) was measured with the question: "How many smoking patients have you assisted by means of the protocol?" Five answer options were given, with scores in brackets: nobody (0), the minority (1), half of them (2), the majority (3), all eligible patients (4).

In addition, the nurses' age, gender, how long they had already worked in this particular ward, the type of ward, the number of working hours a week, whether or not they had attended the training, and their own smoking behawion were regarded as external variables and therefore also measured.

\section{Questionnaire Distribution}

Questionnaires were sent to the internal program co-ordinators $(\mathrm{PCS})$ of the wards involved, who in turn distributed them among the ward nurses. The IPCs, had to inform the researcher beforehand of the number of questionnaires they needed. A letter providing information on the purpose of the survey and the assurance of confidentiality was attached to each questionnaire. The letter also asked nurses to complete the questionnaire individually and to return it within two weeks in an enclosed postagepaid envelope. Participants returning the questionnaire could win a prize of $\$ 25$. If they 
wanted to be eligible for the prize, the nurses had to fill in their names and the name of the hospital in which they worked. These personal details were, however, deleted after the prize had been awarded. Nurses were informed about this procedure.

Postal reminders were sent to the IPCS at two and four weeks after the first mailing, asking them to renind all nurses to return the questionnaire. We did not send postal reminders to non-responding nurses personally, because we had assured their confidentiality. It would therefore have been incorrect to provide the IPCS with the names of the responding nurses and ask for the names of the nurses who had not responded yet. The importance of stimulating nurses to return the questionnaire was also emphasized to the IPCS in one of the meetings that were regularly held with them to monitar the project.

\section{Statistical Analysis}

Data analyses included descriptive statistics of the respondents. Unequal variance Ttests ${ }^{\text {tio }}$ were conducted to investigate differences in the individual attitudinal, social influence, and self-efficacy expectation beliefs between nurses who intended to continue using the protocol and those who did not. For this purpose, respondents were classified into wo groups: nurses with a positive intention toward continued use $(N=61)$ and nurses with a negative intention $(N=24)$. The latter group combined those nurses who were sure that they would not continue to use the protocol and those who reported being unsure about it. Intenders included nurses who stated that they would probably or definitely continue to use the protocol. Differences between intenders and non-intenders regarding the external variables were also assessed by unequal variance T-tests for continuous variables (age, how long nurses had already worked in this particular ward, number of working hours a week). Chi-square tests were employed for the dichotomous variables (gender, type of ward, having attended training), and a Mann-Whitney U test for the ordinal variable level of experience. To assess whether intenders differed from nonintenders in their smoking behavior, a Fisher's. Exact Test was performed, due to the small number of non-intending nusses who smoked $(N=3)$. Furthermore, Pearson correlations were computed between the ASE concepts and nurses" intentions. The ASE model was tested by means of a two step sequential multiple regression analysis (Tabachnick \& Fidell, 2001). In the first step, all attitude, social influence and self-efficacy expectation concepts were entered into the model. This step was employed to assess whether and to what extent the ASE concepts explained nurses intentions to continue using the protocol. The second step assessed whether external variables such as level of experience could provide a significantly better explanation of nurses intentions than the previous model with only the ASE concepts induded. Only external variables that were significantly related to intention were entered here (see the Questionnaires subsection for an over- 
view). Assumptions for regression analysis and a check on multicollinearity beween the independent variables were found to be satisfactory.

\section{Results}

\section{Characteristics of Respondents}

A total of 164 nurses received a questionnaire of whom $85(52 \%)$ returned it. The final sample consisted of 21 men (25\%) and 63 women (74\%); one nurse did not answer the question on gender. The mean age was 36.7 years $(S D=8.44)$. Nurses had already worked an average of 8.35 years $(S D=5.94)$ in their current ward, and worked an average of 31.9 hours $(S D=7.46)$ a week. Data reveled that $54 \%(N=46)$ had never smoked, $26 \%(N=22)$ had smoked at some stage, and 19\% (N=16) were smokers, while the smoking behavior of one nurse was missing.

With respect to nurses" intentions to continue using the smoking cessation proto$\mathrm{col}, 8 \%(\mathrm{~N}=7)$ stated that they probably would not use it anymore, $20 \%(\mathrm{~N}=17)$ were unsure about its continued use, $47 \%(\mathrm{~N}=40)$ indicated that they would probably continue to use it, and $25 \%(\mathrm{~N}=21)$ were convinced they would continue to use it.

Furthermore, $5 \%(N=4)$ reported that they had never used the smaking cessation protocol during the effectiveness study: $25 \%(N=21)$ had used it for some patients; $20 \%(N=18)$ had used it for half of the patients; $40 \%(N=33)$ for the majority of the patients and $11 \%(\mathbb{N}=9)$ for all eligible patients.

\section{Differences between Intenders and Non-intenders}

As mentioned in the Method section, wo groups were created: intenders ( $N=61)$ and non-intenders ( $N=24$ ). The groups did not differ significantly in their demographics, the time they had worked in the particular ward, the type of ward, the number of working hours a week, or having attended training. No differences in intentions were found bewween smoking nurses $(N=16)$ and nonsmoking nurses $(\mathbb{N}=68)$; only 3 smoking nurses did nat intend to continue using the smoking cessation protocol. Intenders had used the protocal significantly more often $(p<.05)$ than non-intenders. Fifty seven percent $(\mathrm{N}-35)$ of the intenders had used it for more than half of the patients compared to $29 \%(N=7)$ of the non-intenders, while $50 \%(N=12)$ of the non-intenders had used it for a minority to half of the patients compared to $21 \%(\mathrm{~N}=13)$ of the intenders.

With respect to the two attitudinal subscales, Table 5.1 shows that nurses in general were moderately convinced of the advantages of the smoking cessation protocol 
and of its simplicty. Table 5.1 also shows that intenders were more convinced than non intenders that the protocol had more advantages than disadvantages. Intenders were also more convinced of the opportunity the smoking cessation protocol provided to improve their preventive performance. Furthermore, they were more convinced of

\section{Table 5.1 Means of and significant differences in attitude, social intluence and self-efficacy scores for imtenders and non-intenders $(N=85)$}

Attitude irems

Oxerall

non-intenders intenders $(N=24)$ $(\mathrm{N}=69)$

Auturtages of the potocal $(-2 /+2)^{2}$

in general, the protocol...

thas more adwantages than disadwantages

is a useful tool to help patients think about the pros of quitting

is a useful toat to motiwate patients to quit smoking

providers me with an opportunity to improve my performance in prevention

helps me to address patienis' smoking behawior in a systematic way

is a useful tool to prevent relapse after discharge

helps me address the topic in an easier and more pleasant way

is more effective in redincing smoking than nomal smoking cessation care

is an improvement compared to the normal smoking cessation approach

emphasizes patients' own responsibillty for their smoking behavior

is congruent with my present working methods

is feasible in daily practice

is flexible and easy to adjust to daily practice

simplicity of the protocol $(-2 /+2)$

The protocol is easy to understand

The protocol is easy to use

I have had enough instruction on the use of the protocol

social infuence items

Discouragement or stapport perceived from .... $(-2 /+2)$ (a)

$0.61 \quad 0.25 \quad 0.75^{\circ}$

1.09

1.59

0.73

0.99

0.52

0.78

0.48

0.74

0.96

0.44

0.68

0.44

0.82

0.62

0.44

overall
0.88

0.25

0.21

0.67

0.33

0.58

0.25

0.50

0.92

0.01

0.01

0.21

0.58

0.01

0.01

non-intenders
1.18

0.72

$0.93^{\circ}$

1. 11

0.59

0.85

$0.57^{\circ}$

0.84

0.98

$0.57^{\mathrm{F}}$

$0.92^{\circ}$

$0.52^{c}$

0.92

$0.90^{\mathrm{s}}$

$0.64^{\text {th }}$

\footnotetext{
- cardiologists

- candrologists
- supervisory staff

colleagues

perceived smoking

Perceived smoking cessation assistance by ..... $(0 /+4)^{\mathrm{s}}$

cardiologists
sipervisony staft
colleagues
cardiologists colleagues

Hems on setf-efficacy expectations.

$\begin{array}{lll}0.96 & 0.83 & 1.02 \\ 1.25 & 1.17 & 1.28 \\ 0.79 & 0.79 & 0.79\end{array}$

How difficult $(2)$ or easy $(+2)$ dlo you find it to use the CMls protocol in generalt 0.29

\begin{tabular}{rrr}
2.73 & 2.92 & 2.66 \\
2.38 & 2.71 & 2.25 \\
2.53 & 2.75 & 2.44 \\
\hline overall non intenders & intenders \\
\hline lit 0.29 & 0.00 & $0.42^{6}$
\end{tabular}

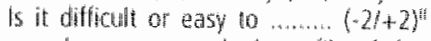

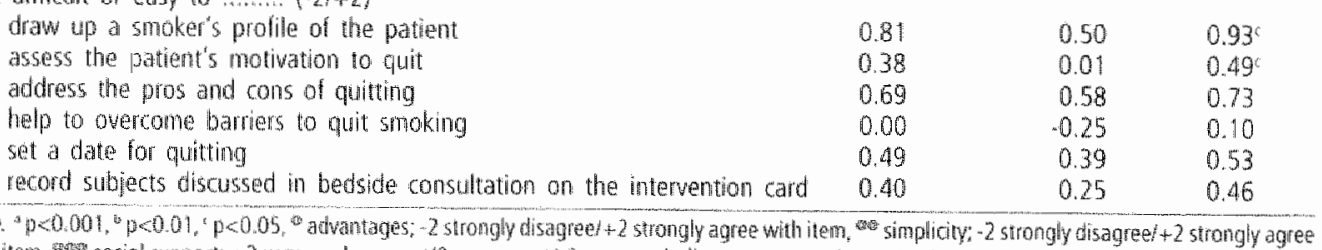

$\begin{array}{lrc}0.81 & 0.50 & 0.93 \\ 0.38 & 0.01 & 0.49 \\ 0.69 & 0.58 & 0.73 \\ 0.00 & 0.25 & 0.10 \\ 0.49 & 0.39 & 0.53 \\ 0.40 & 0.25 & 0.46 \\ \text { percetwed smoking cessation assistance; o no one }\end{array}$

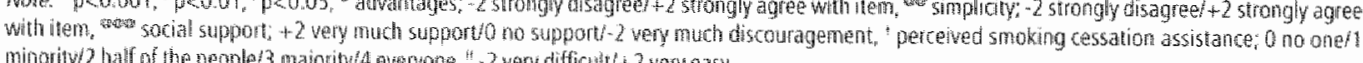

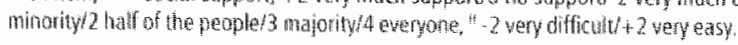

the feasibility of using the protocol in daily practice. Although Table 5.1 also shows other significant differences between intending and non-intending rutrses on the per- 
ceived advantages of the protocol, these differences were all less marked $(p<0.05)$ and are therefore not commented on here. With respect to the perceived simpincity of the protocol, intending nurses differed significantly from non-intending nurses on all the items. Most importantly, intending nurses perceived the protocol to be easier to use than non-intending nurses.

Regarding social influence, Table 5.1 shows that nurses in general experienced some level of suppont from people in their working environment to provide smoking cessation assistance. They also reported that at least half of the people in their working environment provided smoking cessation assistance (also referred to as modeling). However, a comparison of intenders and non-intenders did not reveal significant differences in social support or modeling.

As the Table shows, nurses' selfefficacy expectations were generally moderate and especially low in helping patients to overcome barriers to quit smoking. Intending nurses had a significantly stronger self-efficacy expectation toward using the smoking cessation protocal in general than monintenders did. With respect to the individual tasks defined for nurses in the protocol, intending nurses expressed significantly fewer difficulties in dra* wing up a smoker's profile and in assessing a patient's motivation to quit smoking.

\section{Correlations between Determinants and intended Continued Use of the C-MIS}

Table 5.2 presents the Pearson correlation coefficients between nurses ${ }^{2}$ intentions toward continued use of the smoking cessation protocol and the ASE constructs. Inten" tion correlated most strongly with perceived simplicity of the protocol, followed by the perceived advantages and self-efficacy expectations. Modeling and social support were not significantly associated with nurses' intentions. Furthermore, self-efficacy expectations, advantages and simplicity showed high intercorrelations (Pearson correlation coefficients around, 63), suggesting that the concepts were related. With respect to the external variables (correlations not presented in Table 5.2), intention was only significantly correlated with level of experience $(r=26)$ and nurses' own smoking behavior $(r=.22)$ at $p<.05$. For the non-related external variables, all $p$-values were $>.47$.

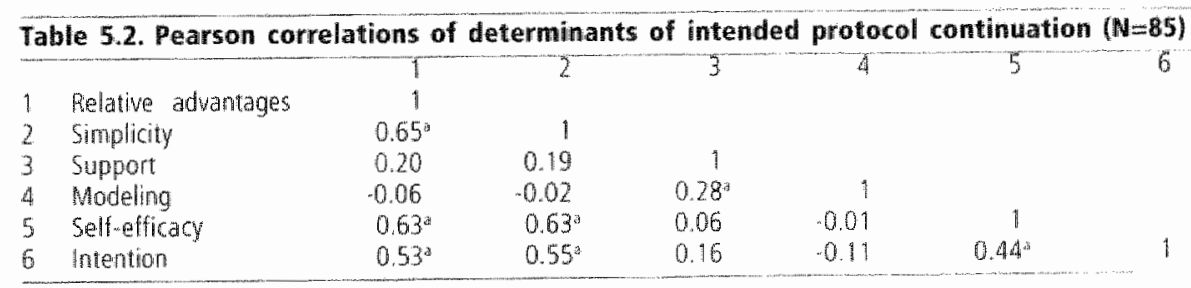




\section{Explanation of Nurses" Intentions toward Continued Use of the C-MIS}

The first step in the regression analysis (entering all ASE constructs) showed that the ASE model explained $38 \%$ of nurses' intentions to continue using the smoking cessation protocol $\left(F(5,77)=9.53, p<.0001, R=0.62, R^{2}=0.38\right)$. However, it was only the attitude subscales which contributed to this explanation, not the social influence and self-efficacy consuructs. The simplicity subscale was the strongest predictor $(B=0.32, p=.01,95 \%$ $C=0.10-0.84)$, followed by the borderline significant advantages subscale $(B=0.25$, $p=05,95 \%, C=-0.01-1.08$ ). Wald statistic $P$-values for the social modeling, social support, and self-efficacy expectation scales were $>24$. Detalled inspection of the partial correlation coefficients of the ASE constructs also indicated that the non-significant factors were not relevant. In the second step, the level of experience and nurses' own smoking behavior were entered. For this purpose, the nurses' own smoking behavior was dichotomized, while four dummy coded variables were created to assess the influence of nurses' level of experience (variable consisted of five categories). This second step showed that neither of these factors added any degree of explanation to the previous model $($ F-change $(5,72)=1.198, p=32)$. The output also showed that the partial correlations between the ASE variables and intention remained unchanged.

In summary, higher perceived simplicity and higher perceived advantages of the protocol were associated with increased intentions toward continued use, while perceived social influences and self-efficacy expectations were not. In agreement with the ASE model, level of experience and nurses' own smoking behavior were mediated by the ASE variables.

\section{Discussion}

The present study analyzed nurses intentions to continue using a protocol to assist cardiac inpatients in smoking cessation. Nurses who did or did not intend to continule using the smoking cessation protocol were compared for their scores on the individual attitudinal, sacial influence, and self-efficacy expectation beliefis regarding the protocol. It was also assessed whether nurses' intentions could be predicted by the ASE model.

Nurses' perceptions of the protocol's simplicity and its percelved advantages explained almost $40 \%$ of the variation in nurses" intentions. Both factors were also found to be important in other studies of the diffusion of health education programs, although they focused on the early adoption or implementation phases (Brink et al., 1995; Mesters \& Meertens, 1999; Parcel et al., 1995; Rogers, 1995). Our finding suggests that perceptions of simplicity and advantages remain important for continued use.

Neither social influence constructs nor self-efficacy expectations contributed to the explanation of nurses' intentions. The lack of contribution of self-efficacy was con- 
trany to our expectations, since it was found to be the strongest predictor in nurses: carewrelated intentions in two other studies (Nash et al. 1993; Vermette \& Godin. 1996. Moreover, low self-efficacy expectations and lack of skills have frequently been reported in the literature as major barriers in the provision of smoking cessation assistance (Buchanan, Huffman, \& Barbour, 1994; Faulkner \& Ward, 1983; Goldstein et al., 1987; Lillington, 1997; Nagle et al., 1999; Orlandi, 1987; Sanders et al., 1986). The Ttest results, however did indicate that intenders had stronger self-efficacy expectations on several activities than non-intenders. Hence, we would recommend increasing the self-efficacy expectations of non-intending nurses, especially in creating a smoker's profile and in assessing a patient's motivation to quit. The literature provides equivocal findings on the role of social influence in providing smoking cessation assistance. One study among physicians showed that they were more likely to counsel patients on smoking if they received staff support (Duncan et al., 1991). However, two studies on other care-related behaviors among nurses did not show any influence of the social enviromment on their performance (Nash et al. 1993; Vermette \& Godin, 1996).

The present study found that neither the level of prior experience with the smoking cessation protocol nor nurses" own smoking behavior influenced nurses' intentions directly if the ASE constructs were taken into account. In line with the assumptions of the ASE model, the impact of these factors was thus mediated by the attitude constructs that explained nurses" intentions. A study of the determinants of continued use of a smoking prevention program by Dutch high school teachers showed comparable findings for prior experience (Dijkstra, 1995). The impact of nurses" own smoking behavior on their attitudes toward providing smoking cessation assistance has been reported in some eartier studies (Adriaanse et al, 1991; Dore \& Hoey, 1988; Goldstein et al. 1987). More recent studies, however, reported no such correlation (Kwiz et al., 1995; Nagle et al., 1999). It is conceivable that nurses" perceptions of their professional performance in providing smoking cessation assistance may have changed over time and have become more independent of their own behavior. However, our findings on the influence of the nurses" own smoking behavior should be interpreted with caution, due to the small number of smokers $(\mathrm{N}=16)$ that were included.

Because perceived simplicity and advantages of the smoking cessation protocol were predictors of nurses" intentions, addressing these factors in a dissemination strategy might facilitate the protocol's continued use. In this respect, the results suggest that it needs to be emphasized that the protocol is easy to use and its use is feasible in daily practice. Changing these perceptions might be achieved by showing nurses examples of protocol use in comparable wards by communicating experiences of nurses who have already worked with the protocol, and by letting them experience protocol use on a trial basis. The results also show that nurses need to become convinced that using the protocol will improve their preventive task performance. This might be achieved by convincing nurses that their efforts can make a difference. Hence, it is 
recommended to provide nurses with feedback on the number of patients who stopped smoking. This has so far been impossible because nurses did not have contact with patients after discharge. It is furthermore recommended to highlight the additional value of the protocol compared to usual care, since the present study, like previous studies, showed that cardiac nurses were not conwinced of the effectiveness of smoking cessation assistance (Buchanan et al., 1994; Faulkner \& Ward, 1983; Orlandi, 1987; Sanders et al., 1986; Wiebing \& Baan, 1997). Believing that these efforts lead to smoking cessation among patients seems a prerequisite for the implementation and continued use of this protocol.

Our study is subject to certain limitations. First, replication of the results in a longitudinal study is needed, since our design precludes conclusions about causes and effects. Second, the results may only reflect the characteristics of those nurses who worked with the smoking cessation protocol. Third, nurses may have provided biased reports since they were expected to have implemented the protocol. Although nurses were asked to report honestly, and to give their own opinions, which would be treated confidentially, responding nurses may still have been indined to exaggerate their intentions, and to underreport their own smoking behavior. Fourth, nurses who were positive about the protocol may have been more likely to respond to the survey than nurses who were not. The low response rate may also have been caused by the fact that we collected the data during the summer vacation period. The response rate may, however, also have been underestimated since IPCs may have asked for more questionnaires than they actually needed". Fifth, the questionnaire did not assess organizational factors. Organizational characteristics (e.g., the size of the organization, its climate, decision making policies, and smoking policies) may also affect continuation (Basch, Eveland, \& Portnoy, 1986; Oldenburg et al., 1997; Orlandi, 1987; Parcel et al.. 1995; Paulussen, 1994; Paulussen, Kok, Schaalma, \& Parcel, 1995; Rogers, 1995; Van Assema et al., 1998). Sixth, it remains unclear whether the attitude subscales reflected two single subsets of items since no factor analyses were employed due to the small sample size. The rellability coefficients (Cronbach's alpha) supported, however, that reliable subscales were formed.

Despite these limitations, the study provided important insights into nurses' individual motives for intending to continue using the smoking cessation protocol. The results can be used to develop a strategy to facilitate the continued use of the protocol in clinical practice in the Netherllands. 


\section{Chapter six}

\section{MAIN CONCLUSIONS AND DISCUSSION}

\section{Abstract}

The subject of this thesis is the development and evaluation of a minimal-contact smoking cessation intervention intended for patients hospitalized with $\mathrm{CHD}$, as well as the factors stimulating or impeding its continued use. The studies described in this thesis were conducted within the framework of the ABC planning model. The aim of this final chapter is to summarize, integrate and discuss the findings of the studies.

The first section summarizes the main findings and conclusions of the studies. The second section reviews their methodological aspects, while the third section includes recommendations for further research. The final section provides recommendations for improvement of the C-MIS, its training, and includes some general recommendations. 


\section{Summary and Discussion of Major Findings}

\section{Why a Smoking Cessation Intervention for Cardiac Inpatients}

The first chapter of this thesis outlined the need to quit smoking for patients with CHD. Although smoking cessation improves the prognosis of these patients more than any other treatment. most patients persist in or resume smoking after a cardiac event (Deckers et al, 1994; Havik \& Maeland, 1988; Hujibrechts et al., 1996; Marshall "1990; Rigotti et al. 1991). Several studies on smoking cessation programs for cardiac inpatients (Condon, 1997; Johnson et al., 1999; Ockene et al. 1992; Orleans et al., 1990; Rigotti et al., 1994; Rosal et al., 1998; Taylor el al., 1990), and evidence-based cinical practice guidelines (Ficre et al. 2000; Raw ef al., 1998; U.S. DHHS, 1996) have shown that it is possible to intervene effectively on smoking. Therefore, it was quite surprising to find that smoking cessation guidance was rarely provided in Dutch cardiology wards (NIPO, 1995: Wiebing \& Baan, 1997\%. It was also surprising that there was no smoking cessation protocol available at Dutch cardiology wards, and none had been tested. To overcome this situation, a smoking cessation protocol (called C-MIS) with which cardiac nurses and cardiologists could assist their patients to quit smoking, was developed and tested for effectiveness (as described in Chapters 3 and 4). The protocol aimed to achieve smoking cessation arnong hospitalized CHD patients, and a $10 \%$ difference between the intervention and the usual care group was estimated to be achievable and clinically relevant. The Minimal Intervention Strategy for general practitioners (called MIS) which had prowed its efficacy in general practice, served as a blueprint for the C-MIS.

Since effective cessation protocols are not yet in place for Dutch cardiac patients, Chapter 1 also indicates the need for research into the determinants of smoking cessation among cardiac inpatients (which are the subject of Chapter 2), and into the motives of cardiac nurses to continue using the C-MIS (discussed in Chapter 5).

\section{Cardiac Inpatients" Phases of Change and Determinants}

First, our findings confirmed that most hospitalized CHD patients are already in the action phase during hospital admission (Kristeller et al., 1992; Ockene et al., 1992). This contrasts with CHD patients visiting the cardiology outpatient dinic of whom only a small proportion are in the action phase (Breteler et al ${ }_{*}$ 1988; Van Berkel et al. submitted-a).

Second, the study showed that patients in the action phase labeled as actors in the stage of change typology) need to be divided into internally and externally motivated patients. Analyses on scale-level showed that the latter group expressed signifi- 
cantly weaker intentions to refrain from smoking, were less convinced of the pros of quitting and more convinced of the cons, perceived less social support and had lower selfwefficacy expectations than internaly motivated patients. Externally motiwated actors showed a pattern of scores on the ASE scales which was comparable to that of contemplators, although their behavior differed. Kristeller and colleagues (1992) already suggested that the group of actors consisted of subgroups. However they categorized patients who had not smoked during hospital admission as actors, contemplators or precontemplators depending on their confidence and desire to remain abstiment. Our findings suggest that externally motivated actors should be considered as a separate group in the stage of change typology. Their behavior differs from that of contemplators, their intentions and most ASE determinants differ from those of internally motivated actors and their intentions, perceptions on cons and smokers in the enviromment differ from those of precontemplators.

Third, the study showed that the relative importance of the psycho-social determinants varied with the phases of change. lt was found that precontemplators, contemplators and internaly motiwated actors generally fitted the $\theta$ pattern with respect to their scores on the ASE scales, while externally motivated actors fitted the $\theta$ pattern as contemplators. The results suggest that the pattern is valid for hospitalized CHD patients. In line with other studies on the $\varnothing$ pattern, we found that social support increases steadily from precontemplation to action, and that intemally motivated actors have higher selfefficacy expectations tham patients in the previous phases (De Vries \& Backbier, 1994; De Vries \& Mudde, 1998; De Vries et al. 1998; Dijkstra el al., 1996). However, in contrast to studies among healthy populations (De Vries \& Backbier, 1994; De Vries \& Mudde, 1998; De Vries et al., 1998; Dijkstra et al., 1996; Velicer, Rossi. Diclemente, \& Frochaska, 1996), we were not able to show that precontemplators perceive significantly less pros of quitting than contemplators, although the scores did provide indications for this. This might, however, be due to the insufficient power of our study, since only a small proportion of the sample were in the precontemplation phase. From another perspective, it could also be that attitude remains unchanged in the transition from precontemplation to contemplation. Malotte and colleagues (2000) found recently that this stage transition is not necessarily preceded by attitude chan* ge. Their research, however, concened condom use and not smoking.

Fourth, more detailled analyses of differences on item level fe.g., separate beliefs on expected pros of the behavior) showed that CHD patients, with the exception of intemally motivated actors, are not convinced of the gains of quitting in terms of reduced heart complaints and reduced risks of rehospitalization. A study among CHD pai" tients in the outpatient clinic setting showed similar findings (Van Berkel ett al., submitted-a). More than a third of these patients stated that they did not know whether their cardiac condition would be improved if they quit smoking. Several other studies, however, reported disease severity and physical complaints as important reasons to quit or as predictors of quitting (Breteler et al., 1988; Glasgow ell al., 1991; 
Goodman et al., 1998; Marshall, 1990; Mckenna \& Higgens, 1997; Ockene et al., 1992). Perceiwed physical complaints were, however, not measured in our study.

In sum, this study provided new theoretical insights into the motivational phases of change and the underlying psycho-social determinants in cardiac inpatients. The firdings concerning the pattern confirm that it is valuable to tallor smoking cessation guidance to patients phases of change in the process of smoking cessation. Hence, the study provided support for the theoretical assumptions of the C-MIS, which tailors cessation assistance to patients' motivational phases of change. The study also showed that the assessment of the phases of change (DiClemente et al., 1991) may need to be adapted for hospitalized populations. Adaptation of this assersment had already been suggested by others, though not specifically for hospitalized patients (Whitelaw, Baldwin, Bunton, \& Flynn, 2000).

\section{The Effectiveness of the C-MIS}

The study aimed to assess the effectiveness of the C-MIS, as well as the predictors of cessation in cardiac inpatients. Regarding the effectiweness, the C-MIS aimed to contribute to a $50 \%$ point prevalence smoking abstinence rate three months after the intervention, compared to an expected $40 \%$ for the usual care. Additional goals with regard to continued abstinence after three and twelve months and point prevalence abstinence after twelve months were not specified in rates. All outcomes were assessed by self-reports in questionnaires.

A Pretest-Posttest experimental design was planned, but turned out not to be achievable due to the demands made by some of the hospitals. This resulted in selfselected assignment of four hospitals and random assignment of the remaining seven hospitals (Pretest-Posttest quasi-experimental design ex post). Five hospitals constituted the experimental group (C-MIS). Six other hospitals served as the control group (usual care). Patients nested in hospitals were the unit of analysis.

With regard to the main outcome, lie, point prevalence abstinence after three months, logistic regression analysis controlling for covariates including baseline differences showed that the intervention was effective. However if dropouts ${ }^{12}(22 \%)$ were treated as smokers, the effect was less pronounced than if they were left out. With regard to continued abstinence, only the analysis without dropouts showed an inter vention effect. Similar analysis of point prevalence abstinence and continued abstinence after twelve months also showed significant interwention effects only if dropouts (27\%) were not included in the analysis. No evidence was found at either of the followup moments that the differences in the randomization procedure (i.e., self-selection by four of the eleven hospitals) had influenced the treatment effects (as an interaction effect) or the smoking cessation outcomes (as a main effect). Furthermore, random 
effect logistic regression analyses failed to show random-hospital effects and confirmed the SPSS analyses in terms of the size and significance of the treatment effects.

The process evaluation among patients in the experimental group showed that they appreciated the way they were approached on smoking. It also showed that not all patients had received the full intervention. About one-fifth of the patients indicated that they had not been exposed to certain components of the in-hospital part of the intervention. About a quarter of the patients indicated that the cardiologist had not addressed their smoking behavior after discharge, while the telephone follow-up (which was initially planned) was rarely applied.

In view of the substantial dropout rate that occurred in the study, which was related to treatment and some baseline characteristics, greater weight was attached to the results of the intention-to-treat analysis (i.e., with dropouts included as smokers). Most other studies among cardiac inpatients have also used intention-to-treat results to judge effectiveness (Johnson et al., 1999; Miller et al., 1997; Ockerie et al., 1992; Rigotti et al., 1994; Taylor et al., 1990). Hence, it must be concluded that the intervention only showed small effects in the short term.

Comparison of our results with those of other studies among cardiac inpatients. may help to explain why the effects were smaller than expected in the short term and no effects persisted in the long term. It might also provide indications for improvement of the intervention. Such comparisons are, however impeded by differences in the methodologies used. Most important, five of the six effectiveness studies found in the literature were randomized controlled trials (Miller et al., 1997; Ockene et al., 1992; Rigotti et al., 1994; Rosal et al., 1998; Taylor et al., 1990). Four of them included biochemical validation (Miller et al., 1997; Ockene et al., 1992; Rigotti et al., 1994; Taylor et al., 1990), while five used intention-to-treat procedures (Johnson et al., 1999; Miller et al., 1997; Ockene et al., 1992; Rigotti et al., 1994; Taylor et al., 1990). Furthermore, most follow-up measurements were conducted after six and twelve months, while our study measured the effects after three and twelve months. All interventions studied were more intensive than ours. Our dropout rate was also higher, which may have led to more conservative effect estimations.

In line with our short-term findings, two studies that also assessed the outcomes in the short term (four or six months of follow-up) reported some behavioral effects (Johnson et al., 1999; Ockene et al., 1992). However, the intervention studied by Ockene and colleagues was only effective for patients with severe Ml, while Johnson and colleagues reported that patients exposed to the intervention were less likely to relapse. These researchers did not find significant intervention effects on smoking cessation, which they attributed to a type $\|$ error (the probability of confirming the effectiveness of an intervention although it is not really effective). Rigotti and colleagues (1994), who used a follow-up period of four months, failed to show intervention effects on (biochemically validated) intention-to-treat point prevalence abstinence. 
Those studies among cardiac inpatients which used follow-up periods of welve months reported their intervention to be effective (Miller et al, 1997; Ockene et al. 1992; Taylor et al., 1990). By contrast, Rigotti and colleagues (1994), who failed to find intervention effects after four months, found no effects after twelve months either. As regards the effectiveness of the interventions. Miller and colleagues (1997) found that repeated telephone support after discharge increased the cessation rate. The study by Dckene and colleagues (1992) reported similar results after six months and twe ve months of follow-up, viz. that the intervention was only effective for patients suffering from severe MI. These effects were, however, not sustained during a five-year follow-up period (Rosal et al., 1998). These three successful interventions were more intensive than ours and included an intensive telephone follow-up guidance for patients. Nicotine Replacement Therapy (NRT) was not included in the interventions studied by others, nor in ours.

The lack of effect after welve months in our study contrasts with the findings of the efficacy study on the MIS, which served as a blueprint for our intervention (Pieterse, 1999). However, similar to our results, another Dutch effectiveness study, applying the MS in the cardiology outpatient clinic (called P-MIS; also failed to find an effect on smoking cessation after one year (Van Berkel et al., submitted-b). These researchers recommended a more intensive program for this population (Van Berkel al al, submitted-b).

Two meta-analyses that included studies with at least five months of follow-up concluded that smoking cessation interventions increase the likelihood of quitting among hospitalized patients (Fiore et al. 1997; Rice \& Stead, 1999). Both recommended to include in hospital behavioral counseling, NRT and aftercare (behavioral reinforcement and relapse prevention) in smoking cessation interventions. In addition, these meta-analyses suggested that several types of heaith care providers need to cooperate.

Several conclusions can be drawn from our study. First, it can be conduded that our intervention is effective in the short term, and our effect estimation may even have been too conservative. A greater short-term effect might have been obtained if the intevention coverage had been complete and had met performance standards. Second, no one-year effects were found, which might be due to several factors: (1) the absence of an intensive program with aftercare aimed at relapse prevention, (2) incomplete implementation of the intervention and (3) overly conservative effect estimations due to the intention-to-treat procedure.

The fact that our intervention (including the aftercare) might not have been intensive enough to produce long-term effects is primarily suggested by the fact that all successful interventions reported in earlier studies were more intensive, induding the after-discharge follow-up, than ours. In addition, it is suggested by the fact that our intervention was able to produce short-term effects, while long-term effects were absent. In this context, it should also be mentioned that the Health Counseling model 
underlying the MIS, C-MIS and P.MIS has recently been criticized for its lack of clarity on how to guide patients in achiewing long-tem behavioral change (i.e. relapse prevention) (Mesters et al. in press). A meta analysis of controlled trials of patient education among CHD patients also indicated that the absence of explicit feedback or reinforcement of a new behavior is related to the lack of long-term behavioral change (Mullen et al., 1992).

With respect to our second explanation for the absence of long-term effects in our study, (i.e. incomplete implementation) several aspects need to be addressed: (a) not all patients received the full intervention, (b) the in-hospital part of the C-MIS was administered by ward nurses who were mostly trained by the researchers, but had to incorporate the intervention into their daily ward program. In other effective interventions, however, it was research nurses or professional counselors who administered the interventions, which means that they were probably administered better and in a: more standardized manner Uohnson et al., 1999; Miller et al., 1997; Ockene et al. 1992; Rigotti et al., 1994; Taylor et al., 1990). Although we have also made efforts to ensure quality control and standardized delivery (e.g., by assigning internal program co-ordinators to supervise the interwentions and by training nurses), it is conceivabie that these aspects need improvement. (c) Cardiologists and general practitioners (GPS), who had to provide aftercare, were not trained in the use of C-MIS nor in relapse prevention techniques. GPS were not trained because they were assumed to be familiar with the protocol, while the cardiologists were not trained because Stivoro wanted to assess whether it would be enough to provide them only written instructions, since this would make large scale implementation in the future easier. Furthermore adequate aftercare might have been hindered by the way both health care providers were approached to perform aftercare. (d) The process evaluation revealed that organizational barriers (ward shifts, very busy schedules) occurred, which may have affected the quality and level of implementation of the C-MIS. In this respect it also needs attention that ward-managers and most nurses disagreed with the implementation of the telep. hone follow-up. Reasons were that it would take to much time and that patients would change the subject to issues related to their disease (which would be hard to avoid). These perceptions were hard to change at the time of the effectiveness study, but need attention in future.

Our third explanation concerns the high dropout rate (27\%), which was considerably higher than in other studies among hospitalized CHD patients (Johnson et al., 1999: Miler et al., 1997; Ockene et al., 1992; Rigotti et al., 1994; Taylor et al., 1990). As a result, our effect estimation may have been too conservative. Unfortunately, this issue could not be clarified from the available data. 


\section{Determinants of Cessation}

An additional goal of the effectiveness study was to analyze the determinants of cessation. For this purpose, information was obtained on the baseline characteristics associated with patients' smoking status after three and twelve months. The predictors differed slightly beween the outcomes (point prevalence abstinence and continuous abstinence) and the follow-up moments. Furthermore, intention-to-treat analyses revealed slightly different predictors. The greatest weight was attached to the results of the analyses that did not include dropouts as smokers, because the sub. stantial dropout rate that occurred in the study was related to some baseline characteristics, which in turn may have led to misclassification of smokers in the intention-to-treat analyses. Seweral other studies that assessed predictors of smoking cessation among cardiac inpatients also used this procedure, for the same reasons (Ockene et al., 1985; Rigotti et al., 1994; Rigotti et al., 1991; Rosal et al., 1998; Van Berkel et al., 2000; Van Berkel et al., submitted-b; Greenwood et al., 1995).

After three months of follow-up, point prevalence abstinence was predicted by the determinants admission for MI, being an internally motivated actor having strong selfefficacy expectations and having made the first attempt to quit in the year prior to admission. Continuous abstinence was only predicted by first admission for CVD and by being an internally motivated actor. After one year of follow-up, it was strong baseline intentions to quit, strong self-efficacy expectations and perceiving fewer smokers in the social environment (modeling) which predicted point prevalence abstinence. Continuous abstinence was predicted by first admission for CVD, higher level of education, admission for $\mathrm{Ml}_{4}$ being an internally motivated actor, perceiving fewer smokers in the social environment and strong self-efficacy expectations. Because these factors were independently related to the smoking status, patients who were characterized by more of these factors were more likely to be abstinent at follow-up. Most of these factors had been reported earlier as predictors among CHD patients (Ockene et al., 1992; Orleans et al., 1990; Rigotti et al. 199.4; Rigotti et al., 1991; Rosal et al., 1998; Taylor et al., 1990; Taylor et al., 1996; Van Berkel et al., 2000; Van Berkel et al., submitted-b; Greenwood et al., 1995). However, it might be argued that significance levels of our tests were not adjusted in order to decrease the probability that significant differences would occur merely by chance due to multiple testing. Application of a Bonferroni type adjustment for both follow-up moments separately (i.e., $p<.003: p=.05 / 17$ cowariates) would only have shown self-efficacy expectations as a predictor of point prevalence abstinence after three months. No other predictors would have been significant related to the outcomes. Most studies that reported on the predictors of smoking cessation among CHD patients, however, did not correct for multiple testing, which impedes comparison.

In sum, the studies presented in Chapters 3 and 4 provided new practical insights into the application of the HC model for cardiac inpatients. They showed that the HC 
model can be applied successfully to achieve smoking cessation in cardiac inpatients, although it may only result in short-term effects. The studies also showed that it is necessary to provide extensive guidance for the health care professionals involved, to help them work with the protocol, and to supplement the HC model and the C-MIS with adequate relapse prevention techniques. The studies also contributed theoretical insights into the factors associated with smoking cessation in cardiac inpatients, which is also relevant for dinical practice.

\section{Cardiac Nurses' Intentions and Motives to Continue Using the C-MIS}

To facilitate the continued use of the C-MIS in a non-research setting (under condition that the C-MIS is effective), cardiac nurses' intentions and their underlying motives to continue using the protocol were studied within the theoretical framework of the ASE model. The survey (described in Chapter 5) was conducted among nurses of the wards that served as the experimental group in the effectiveness study, one week after the interventions for the effectiveness study had been concluded.

The most important finding was that nurses' perceptions of the protocol's simplicity and its perceived advantages explained almost $40 \%$ of the variation in nurses' in. tentions for continued use. This is quite high compared to other studies that applied the Theory of Planned Behavior (which is comparable to the ASE model) to explain and predict health related behaviors (Godin \& Kok, 1996). The predictors we found have also been reported in several other studies on the diffusion of health education programs, although they focused on the earlier adoption or implementation phases (Brink et al. 1995; Mesters \& Meertens, 1999; Parcel et al., 1995; Rogers, 1995; Steckler et al. 1992). Our findings suggest that perceptions of simplicity and advantages of a pro* gram remain important for continued use as well. Furthermore, the study showed that most nurses were not convinced of the effectiveness of the protocol or of their own ability to provide smoking cessation assistance appropriately. This is in line with previous findings (Buchanan et al., 1994; Faukner \& Ward, 1983; Nagle et al., 1999; Orlandi, 1987; Sanders ell al., 1986; Wiebing \& Baan, 1997).

In sum, the results of this study contribute to theoretical insights into psycho-social factors that stimulate or impede nurses' intentions for continued use of the protocol. It also provided new practical information that can be used by Stivoro to develop a strategy to facilitate (continued) use of an improved version of the protocol in clinical practice in the Netherlands. 


\section{Methodological Issues}

\section{Cardiac Inpatients' Phases of Change and Determinants}

The findings of the study on the motivational phases of hospitalized CHD patients and their underlying psycho-social determinants need to be interpreted with caution, mainly due to its cross-sectional mature, which precludes conclusions about causes and effects. A related problem concerns the way patients were dassified within the motivational phases of change. Furthermore, the results concerning the precontemplators need to be interpreted with caution due to the small number of patients included in this motivational phase.

With respect to the external validity of the results (i.e., the extent to which the observations in the study can be generalized to other settings and populations with similar (haracteristics), it needs to be emphasized that generalizations to the whole population of CHD inpatients have to be made with caution. This is mainly due to the fact that it is unclear whether participants to the studies differed from patients who were not willing to participate or were not asked. The long inclusion period and the information gathered among internal program ca-ordinators suggested that not all potential patients were approached for participation. Although the internal program co-ordinators indicated that the non-inclusion of patients was unrelated to patient characteristics, selection bias may have occurred. Furthermore, we were unable to assess the characteristics of refusers because the medical ethics committees of the hospitals did not allow us to interview refusers. Another generalization issue concerns the timing of the measurements. The measurements were conducted within 96 hours after admission. The literature shows that cardiac inpatients experience extremely high levels of anxiety and cognitive impairment in the early days of their hospital stay (Sarafino, 1998; Shaw et al., 1987; Townes et al., 1989). They may therefore be strongly motivated to quit smoking at that time. It is, however, conceivable that patients become less motivated to quit when they feel better and are less anxious. Since hospital admission is often short (commonly 6-8 days), motivational phase and underlying determinants may change shortly after discharge from the hospital. It is unclear to what extent the phases of change and the opattern of determinants found in our study can be replicated when measured shortly after discharge. This may have practical implications for aftercare.

\section{The Effectiveness Study}

Although the internal validity of the effectiveness study (i.e., the extent to which the observed effect can be attributed to the intervention) may have been affected by 
several factors, some of these have been successfully addressed by applying appropitate statistical methods. Mast importantly, as mentioned previously, the randomization problem was addressed by the inclusion in the final regression models of a factor for self-selection of treatment condition and its interaction with treatment. The problem of the nested design (i.e., the unit of analysis (patients) differed from the unit of assignment to treatment (hospitals)). was dealt with by performing additional multi-level analyses. Furthermore, possible covariates including baseline differences were controlled for. Finally, our conclusions on effectiveness were based on the intention-totreat analyses. Hence, we accommodated for the selection that occurred in the follow-up.

The main problem as regards the effectiveness of the intervention, however, was that the observed treatment effects in the analyses without dropouts were subject to selection bias, while the observed treatment effects in the intention-to-treat analyses could have been masked due to the large number of dropouts. Furthermore, it is questionable whether the effectiveness study had enough power to counteract the above problems. Most importantly, this might be due to the fact that possible interactions and the nested design were ignored in our power calculations. The validity of the findings of the effectiveness study was furthermore limited by the fact that the present results were obtained under conditions of moderate intervention coverage. Moreover, the process evaluation suggested that the expected quality of administration of the intervention was not always achieved. It is therefore interesting to know whether the results would have been different if complete intervention coverage and adequate quality standards had been achieved. We have tried to address this problem by running secondary logistic regression analyses including additional factors that could provide an indication of intervention coverage and quality of performance. For this purpose, we constructed the factors "good versus bad implementation" and "have or have not spoken with the nurse about smoking". The first reflected the opinion of the researcher with regard to the way wards organized and performed the interventions. The second reflected the answers of patients to a process evaluation question included in the posttest after three months. In contrast to the results on continued abstinence after three months obtained in the logistic regression analyses that did not include these factors, the factor "good versus bad implementation" significantly predicted intentionto-treat continued abstinence after three months. Results with respect to the other outcomes, however, remained unchanged. The factor "have or have not spoken with the nurse about smoking" was unrelated to all outcomes (Bolman \& De Vries, 1999). The validity of this "ad hoc" procedure may, however, be doubted because adjustment for postrandomization variables such as process data disrupts the randomization and may introduce serious biars in the estimation of the treatment effect and testing (see for example, Robins \& Greenland, 1994). Therefore, these secondary analyses were not reflected in our condusions. 
A final remark conceris the lack of validation of the self-reported smoking behavior. Athough effors were undertaken to overcome this deficiency by using an identification coding system, by assuring patients anonymity and confidentiality, and by the application of a bogus pipeline procedure (applied in the 12-months follow-up) (Hansen et al. 1985; Lichtenstein \& Glasgow, 1992; Velicer et al. 1992), overestimation or underestimation of the smoking behavior may have occurred. Several intervention studies, however, have reported that treatment groups are not more likely to misrepresent their smoking behavior than controls (Glasgow et al. 1993; Kottke et al., 1988; Murray et al., 1993). Intervention studies among CHD patients suggest the same (Miller et al., 1997; Ockene et al., 1992; Taylor et al., 1990; Van Berkel et al., submittedb; Greenwood et al, 1995). Therefore, we assume that any bias that might have occurred has not affected the differences found beween the experimental and control groups.

Some factors may have affected the external validity of the study, and may endanger the generalizability of the results. First, some hospittals were not willing to participate in the study. The reasons reported for refusal concerned issues such as mergers between wards and hospitals, and changes in nurse-delivery systems. However, it cannot be excluded that hospitals in which smoking cessation assistance was problematic were less eager to participate. Second, the long period needed to include sufficient numbers of patients suggests that not all smoking patients were approached. Nurses confirmed this, mentioning time constraints and forgetfulness as the most important reasons. As a result, the target population may not have been fully reflected in the sample.

\section{Cardiac Nurses' Intentions and Motives to Continue Using the C-MIS}

Several methodological issues of the study among nurser deserve attention. As regards the internal validity of the study, it needs to be emphasized that the crosssectional nature of the study predudes conclusions about causes and effects. Second. nurses who were positive about the protocol may have been more likely to respand than nurses who were not. Third, nurses may have provided biased reports, since they were expected to have implemented the protocol. Fourth, it is passible that some significant differences might have occurred merely by chance because we did not correct for multiple testing.

With respect to the external validity of the study, it should be mentioned that the results may only reflect the characteristics of those nurses who worked with the pratocol,

Furthermore, the study allowed no condusions to be drawn about the influence of organizational factors that might also influence nurses" intentions toward continued use of the protocol. As suggested in the literature, organizational characteristics le.g., the size of the organization, its working climate, decision making policies, and smo- 
king policies) may a so affect continuation (Basch et al. 1986; Oldenburg et al., 1997; Orlandi, 1987; Parcel et al. 1995; Paulussen, 1994; Paulussen et al., 1995; Rogers, 1995; Van Assema et al., 1998).

\section{Recommendations for Further Research}

Although the studies presented in this thesis provided answers on important issues, warious other issues remain undear, and further research into them seems warranted.

\section{Cardiac Inpatients" Phases of Change and Determinants}

First, with respect to the motivational phases and underlying determinants in cardiac inpatients, it is recommended to perform a longitudinal study on the motivational phases (including the phase of behavioral maintenance), the processes of change and the psycho-social determinants. Such a study would need to show which changes in psycho-social determinants precede the forward or backward transition along the phases of change. In addition, it might be useful to assess whether particular personality factors are related to the phases and underlying determinants. The latter is suggested by the fact that CHD patients' persistence in smoking is related to anxiety and depres sive feelings (Huijbrechts et al, 1996; Ockene et al., 1985).

Second further research should be started into the stability of patients' motivational phases of change and underlying determinants, from admission to a few weeks after discharge. The fact that patients' preparedness to quit probably falls after discharge from the hospital can be deriwed from studies among cardiac outpatients, which showed that only a small proportion of cardiac outpatients are willing to quit in the short term or are already in the action phase (Breteler et al., 1988; Van Berkel et al. submitted-a).

Third, it is recommended to study why subpopulations of cardiac inpatients are not convinced that smoking cessation would improve their chances of being cured and would help prevent further deterioration of their cardiac condition. This might be duse to a cognitive dissonance process (Festinger, 1976). Other explantions might be that patients simply do not know or understand that smoking cessation improves the cardiac condition, that they underestimate its positive effects, or that they underestimate the threat of cardiac deterioration in themselves. The answer to this question could provide suggestions for improwing smoking cessation interventions for patients with smokingrelated diseases. 


\section{Effectiveness of the C-MIS}

Several aspects of the effectiveness of the C-MIS need further study. First, research is needed to analyze factors associated with relapse in CHD patients in order to strengthen the C-MIS as a tool for preventing relapse. While relapse has been thoroughly studied among general populations (Becona \& Vazquez, 1997; Irvin, Bowers, Dunn, Q Wang, 1999; Ockene et al., 2000), only two, rather dated studies have reported on factors associated with relapse in CHO patients (Shiffman, 1982; Simon, Browner \& Mangano, 1992). The recently published review by Ockene and colleagues suggests that a follow-up period of at least wo years should be used to obtain a complete picture of the relapse curve (Ockene et al., 2000). Although the literature mentions a number of recommended techniques for relapse prevention (e.g., telephone counseling, nicotine replacement therapy, training in identifying and coping with high-risk situations, and booster sessions), results of relapse prevention interwentions have been mixed (Becona \& Vazquez, 1997; Curry \& McBride, 1994; Irvin et al., 1999; Ockene et al., 2000) and have not been specifically studied in CHD populations.

Second assessment is needed of effective methods for aftercare including the roles of health care providers. Several researchers have demonstrated that an intensive telephone follow-up increases the effectiveness of a smoking cessation intervention (DeBusk et al., 1994; Miller et al., 1997; Taylor et al., 1990; Taylor et al., 1996). The telephone follow-up in our study was, however, not implemented successfully. Therefore, it should be investigated which circumstances would make it feasible for Dutch nurses or other health care workers to provide telephone support shortly after hospital discharge. The need for support shorty after discharge is indicated by the fact that relapse is most likely to occur within three weeks after quitting 10 ckene et al., 2000; Zhu et al. 1996), while the control visit at the outpatient dinic cardiology usually takes place later. If a telephone follow-up is not possible, a substitute has to be sought and tested for the weeks following discharge. One option might be to make use of computerized tailoning, in which people receive written personal support. Such written support should emphasize the high-risk situations that a patient has indicated, for example in a questionnaire on high-risk situations. Several studies have suggested the efficacy of such interventions in general populations (Dijkstra et al., 1998a; Dijkstra et al., 1998b; Velicer et al., 1993). Further research on aftercare also needs to address the roles of cardiologists and general practitioners. Because their roles were insufficiently addressed in the present research and seemed to leave room for improvement, further research should examine what kind of smoking cessation guidance they are willing and able to provide, which psycho-social factors influence this, and how continuity of care can be achieved among both types of professionals, as well as continuity between the in hospital part of the C-MIS and the aftercare. A final aftercare issue that needs further investigation is the coordination with cardiac rehabilitation programs. Because a substantial part of CHD patients attend a cardiac rehabilitation program after dis. 
charge, it should be studied how such a program can be coordinated with the smoking cessation activities administered in the hospital.

Third, more research is needed into the additional value of medication. It is recommended to study whether it would be useful to prescribe NRT and/or bupropion hydrochloride (e.g., Zyban) in the C-MIS for CHD inpatients or subpopulations thereof. At the start of the effectiveness study, cardiologists were unwilling to prescribe NRT because of the possible adverse cardiovascular effects (Benowitz \& Gourlay, 1997). Meanwhile, studies on NRT for cardiac inpatients have shown that it can be used safely (Haustein, 1999; Tonstad, 1999) and that it doubles the rate of successful quitting in the general population (Fiore et al., 1997; Raw et al., 1998; U.S. DHHS, 1996). At present, however, it is still not common practice to prescribe NRT. A recently published study among a general population showed that treatment with Zyban (which is currently registered as a medication for smaking cessation), resulted in higher long-term abstinence rates and less severe withdrawal symptoms than the use of a placebo (Jorenby et al., 1999). Another study found the same positive effects of this drug in smokers with and without a history of depression (Hayford et al., 1999). Although it is recommended to assess the additional effectiveness of the C-MIS if these medications are included, a problem will arise if such an additional effectiveness is proved. As yet, NRT and Zyban are not covered by health insurance in the Netherlands. If patients would have to pay for it themselves, it might lead to the situation in which not a!l patients who need it would use it.

Fourth, more research is needed to analyze the impact of more intensive counseing. Research into the need for more intensive counseling is suggested by the fact that our intervention only showed minor effects in the short term and was ineffective in the long term. All successful interventions referred to in the literature included in-hospital counseling which was at least twice as long as ours (Johnson et al., 1999; Miller et all., 1997; Ockene et al., 1992; Taylor et al., 1990). It is, however, undear how the counseling would have to be intensified. Van Berkel and colleagues found in their review on the impact of smoking interventions in coronary patients that interventions are more effective if they include additional group sessions and support devices (Van Berkel et all, 1999b). Therefore, it might be valuable to intensify the intervention with these elements.

Fifth, more research is needed to analyze how smoking patients who suffer from psychological problems (e.g., anxiety and depressive feelings), which is estimated to apply to one-fifth of the CHD population (Falger et al., 2000), can be appropriately addressed. High state anxiety levels and depression are associated with persistent smoking (Huijbrechts et al., 1996; Ockene et al., 1985; Van Berkel et al., 2000). If cigarette smoking is perceived as a way to cope with these feelings, it is unlikely that these patients will quit successfully. They first have to deal with their problems in another way. This might be achieved by psychological treatment. It might also be valuable to combine psychological treatment with medical prescription of anti-depressants (e.g., Bupropion hydrochloride, which was originally an antidepressant, but was recently re- 
gistered as a medication for smoking cessation). Furthemore, as has already been suggested by others (Van Berket et al., 2000), it might be useful to supplement the protocol with an assessment of the psychological profile of patients who smoke. Recently, Falger and colleagues (2000) recommended such an assessment of all CHD inpatients. This would allow the identification of depressive smoking patients so they could be providad with extra support.

In sum, further research should initially aim to identify what is possible and feasible in current cardiology practice with regard to an intensification of the C-MIS. Second, it should be tested whether an extended version of the protocol (including more intensive tools for subpopulations with psychological problems) would lead to better outcomes in terms of sustained abstinence from smoking. Since the C-MIS has already proved to be effective in the short term; and it is conceivable that greater effects could be achieved if intervention coverage is complete and the intervention is administered appropriately, it would seem useful to conduct a well-designed effectiveness study comparing usual care, the minimal version of the C-MS (i.e., the version studied in the present research project) and an extended version.

\section{Implementation and Continuation of the C-MIS}

As regards the implementation and continuation of smoking cessation guidance in cardiology wards, further study should show what is required at the micro (health care providers), meso (ward and hospital) and macro (national health care policies) levels to ensure that smoking cessation guidance becomes an appropriate standard of care. Several researchers and guidelines recommend simultaneous interventions at each of these three levels (Fiore et al., 1997; Orleans et al., 1993b; Orleans \& Ockene, 1993; Raw et al., 1998; Stewens, Glasgow, Hollis, Lichtenstein, \& Vogt, 1993; U.S. DHHS, 1996). A randomized controlled trial has shown that physicians were more likely to counsel patients on smoking when they received staff support in addition to a training on smo. king cessation counseling (Duncan et al., 1991). The American Agency for Health Care Policy and Research issued the general recommendation that hospital administrators should ensure that education, resouices and feedback are provided to health care workers, in order to promote the implementation of smoking cessation interventions (U.S. CHHS, 1996). It seems likely that health care providers would try harder to influemce the smoking behavior of patients if this were regarded as an appropriate standard of care by hospital administrators and national health care administrators as well. Further research should also study the factors that may contribute to the adoption, implementation and institutionalization of the protocol (after it has beem proved effective) among wards that are unfamiliar with the protocol at present. Interesting would also be to study whether and how nurses' own smoking behavior influences nurses" smoking practice attitudes, intentions and behavior. This because we found a positive 
association between murses own smoking behavior and their intentions to continue using the protocol. Although Nagle and colleagues (1999) reported a comparable finding, a Dutch study among nurses (Wiebing \& Baan, 1997) suggested the opposite. Because of the small number of smokers included in our study, it is recommended to study this relationship with sufficient smoking nurses.

\section{Recommendations for Improvement of the C-MIS}

\section{Recommendations for the C-MIS Protocol}

First, assessment of smoking behavior is not yet current practice for cardiologists and nurses in cardiology wards. As recommended in the smoking Cessation Cinical Practice guideline, identification and documentation of smoking habits need to be included in the C-MIS and in the standard hospital intake procedure carried out by nurses (Fiore et al, 2000). In our effectiveness study, this was done prior to the start of the intervention.

Second, our first study (see Chapter 2) demonstrated that externally and internally motivated actors need to be approached differently. Hence, the protocol has to be sup. plemented with instructions for health care providers to identify these groups. For this purpose, step two of the protocol (assessing the smoking behavior and motivation to quit) needs to be adapted. Health care providers have to learn that externally motiwated patients have to become internally motivated first. As chapter 2 showed that internally motivated patients perceive more pros of quitting and have higher self-efficacy expectations, health care providers need to target both in the intervention for externally motivated actors.

Third, since most smokers were not fully aware of the relationship between their own (persistent) smoking behavior and their recovery (see Chapter 2), nurses" conversations with patients in step three of the C-MIS need to emphasize this relationship. Nurses (possibly helped by cardiologists) have to make efforts to increase patients' awareness of this relationship and need to verify whether patients are really convinced.

Finally, the self-help guide that was provided in the C-Mis needs to be adapted for the specific population of CHD patients, since it was developed and tested for use in general practice. 


\section{Recommendations for the Training}

The effectiveness study (Chapters 3 and 4) and the study among nurses (Chapter 5) suggest that the training for nurses needs improvement.

First, and most importantly, the training should inclucle better guidance in assessing a patient's readiness for quitting, and in assisting patients to find and learn adequate coping strategies to overcome high-risk situations. Several researchers have already reported that training should emphasize the latter (Kviz et al., 1995; Nagle et al. 1999). Bandura suggests that modeling with guided enactment is a powerful tool to increase self-efficacy expectations and the skills that are necessary to perform a particular behavioi (Bandura, 1986). Within this modeling with guided enactment approach, skills that nurses need should be divided into subskills that are easy to perform. Nurse should also be provided with good examples of how to carry out the steps in the protocol (modeling). Valuable tools for this purpose might include a videotape or CD-Rom in which the protocol is explained and carried out step by step by models. What is also needed is guided enactment in easy and difficult situations, followed by professional feedback. Although we used a comparable approach in our training, the results indicate that it needs to be improved.

Second, the training should emphasize that smoking cessation guidance is effective in helping patients to quit smoking and is feasible. For this purpose, nurses have to receive new convincing information on the effectiveness and feasibility of the intervention (Eagly \& Chaiken, 1993; Maibach \& Parrott, 1995; McQuire, 1985), which can be derived from most pubished effectiveness studies, including ours, as well as from published meta-aralyses and from evidence-based clinical practice guidelines.

Third, because training needs to be intensified, and because experience in providing the training has shown that a twa-hour training is too short, it is recommended to lengthen the training, while it might also be valuable to include booster sessions. It is also recommended to create several opportunities for specific training on protocol usage. It might for example be necessary to offer group trainings at several moments during the year, combined with a take-home self-instructive video for nurses who are unable to attend group training.

Fourth, it is recommended to provide cardiologists with group training. In the effectiveness study presented in this thesis, they only received written instructions. A review of the effects of group training in providing smoking cessation interventions among health care professionals (most general practitioners) in primary care settings has shown, however, that group training improves their performance in providing appropriate smoking cessation assistance. Training attendance even predicted smoking cessation among patients (Lancaster, Silagy, Fowler, \& Spiers, 1999). For general practitioners, specific C-MIS training may be less necessary because the National Association of General Practitioners (LHV) already provides GP5 with training in the application of the Mis. It should, however, be investigated whether the LHV would be 
willing to include the CMIS in their training program (a large proportion of the GPS still await training). After all, $\mathrm{CHD}$ patients also visit their family doctors. In this context, it needs to be communicated to GPs that the C-MIS is initiated in the hospital and needs to be continued in GP visits, for which they can use their existing knowledge and the skills needed to administer the MIS. That many GPs still need to be trained can be derived from the fact that by the end of 1999, an estimated $24 \%$ of the Dutch GPs had attended MIS training (Duistermaat, 1999), while an estimated 21\% were applying the MIS at that time (Leest, Hulscher, Tsang, Schattenberg, \& Grol, 1999).

Finally, to facilitate continued use of the protocol, it is important that nurses receive feedback on their performance. For this purpose, training needs to incorporate booster sessions in which nurses can share their experiences in working with the protocol and can get some additional practice.

\section{General recommendations}

It needs attention that the implementation of an (improved) smoking cessation intervention for cardiac inpatients is desirable and still in progress at the time of writing the present thesis. To make sure that an improved version of the C.MIS protocol and its training fit the hospital practice and will be supported by the target groups involved, the linkage group as mentioned in chapter one should continue her work. It needs to be extended with representatives of cardiologists, GPs and cardiac rehabilitation programs. It might also be useful to ask representatives of ward-managers for their co-operation since they have to approve the implementation of al smoking cessation protocol. 


\section{References}

Adriaanse, H., Van Reek, J., Zandbelt, L., \& Evers, G. (1991). Nurses' smoking worldwide. A review of 73 surveys on nurses" tobacco consumption in 21 countries in the period 1959-1988. International Journal for Nursing Studies, 28(4), 361-75.

Ajzen, 1. (1991). The theory of planned behavior. Organizational Behavior and Human Decision Processes, 50(2), 179-211.

Bandura, A. (1986). Social foundations of thought and action: A social cognitive theory. Englewood Cliff: Prentice Hall.

Bandura, A. (1997). The anatomy of stages of change [editoria|]. American Journal of Health Promotion, 12(1), 8-10.

Bartholomew, L. K., Parcel, G. S., \& Kok, G. (1998). Intervention mapping: a process for developing theory- and evidence-based health education programs (see comments]. Health Education and Behavior, 25(5), 545-63.

Basch, C. E., Eveland, J. D., \& Portnoy, B. (1986). Diffusion systems for education and learning about health. Family and Community Health, 9(2), 1-26.

Becona, E., \& Vazquez, F. L. (1997). Does using relapse prevention increase the efficacy of a program for smoking cessation? An empirical study. Psychological Reports, $8 \pi(1), 291-6$.

Benowitz, N. L., \& Gourlay, S. G. (1997). Cardiovascular toxicity of nicotine: implications for nicotine replacement therapy. Journal of the American College of Cardiology. 297), 1422-31.

Block, P. C. (1990). Restenosis after percutaneous transluminal coronary angioplasty: anatomic and pathophysiological mechanisms. Strategies for prevention. Circulation, 81( Suppl. 3), IV2-4.

Boekema, A. G., Kooi, L., Seydel, E. R., \& Pieterse, M. E. (1990). Rapportage implementatie project "stoppen met roken". Een kwalitatieve analyse van randwoorwaarden en uitvoerbaarheid. Report on the implementationproject "smoking cessation". A qualitative analysis of preconditions and feasibility] Enschede: IKA Stedendriehoek Twente/Universiteit Twente.

Bolman, C, \& De Vries, H. (1998). Psycho-social determinants and motivational phases in smoking behavior of cardiac inpatients. Preventive Medicine, 27, 738-47.

Bolman, C., \& De Vries, H. (1999). Effectiviteit van de Minimale Interventie Strategie stoppen-met-roken Cardiologie (C-MIS). (The effectiveness of the Minimal intervention Strategy on smoking cessation for Cardiac Inpatients. Maastricht: Capaciteitsgroep Gezondheidswoorlichting.

Bolman, C., De Vries, H. \& Van Breukelen, G. (in press) Evaluation of a nurse-managed minimal-contact smoking cessation intervention for cardiac inpatients. Health Education Research.

Bracht, N. (Ed.). (1999). Health promotion at the community level 2, new advances. Thoasand Oaks, London, New Dehi: Sage Publications. 
Breteler, M. H. M., Mertens, E. H. M., \& Rombouts, R. (1988). De motivatie tot verandering van rookgedrag: modeltoetsing bil patiënten met hart en longklachten. The motivation to change smoking behavior: modeltesting in patients with heart and lung complaints]. Thidschrift voor Alcohol en Drugs, 14(1), 22-31.

Brink, S. G. Basen-Engquist, K. M., O'Hara-Tompkins, N. M., Parcel, G. S., Gottleb, N. H., \& Lovato, C. Y. (1995). Diffusion of an effective tobacco prevention program. Part 1: Evaluation of the dissemination phase. Health Education Research, 103), $283-95$.

Brug, J, \& Van Assema, P. (1995). Factors differentiating between stages of change for fat reduction. Appetite, 24, 296.

Brug, J., Hospers, H. J., \& Kok, G. (1997). Differences in psychosocial factors and fat consumption between stages of change for fat reduction. Psychology and Health. 12(5). $719-727$.

Bryk, A. S., \& Raudenbusch, S. W. (1993). Hierarchical Linear Model. Newbury Park (Calif): Sage.

Buchanan, C. R., Huffman, C., \& Barbour, V. M. (1994). Smoking health risk. Counseling of psychiatric patients. Journal of Psychosocial Nursing, 32(1), 27-32.

Burling ${ }_{r}$. A. Singleton, E. G., Bigelow, G. E., Baile, W. F. \& Gottlieb, S. H. (1984). Smoking following myocardial infarction: a critical review of the literature. Health $P_{S y-}$ chology, 3(1), 83-96.

Cameron, A. A., Davis, K. B., \& Rogers, W. J. (1995). Recurrence of angina after coronary artery bypass surgery: predictors and prognosis (CASS Registry). Coronary Artery Surgery Study. Journal of the American College of Cardiology, 26 4), 895-9.

Carlsson, R., Lindberg, G., Westin, L." \& Israelsson, B. (1997). Influence of coronary nur. sing management follow up on lifestyle after acute myocardial infarction. Heart, $77(3), 256-9$

Clark, J. M., Haverty, S., \& Kendall, S. (1990). Helping people to stop smoking: a study of the nurse"s role. Journal of Advanced Nursing, 15(3), 357-63.

Conner ${ }_{n}$ M. \& Norman. P. (Eds.). (1996). Predicting heath behaviour: research and practice with social cognition models (1st ed.). Buckingham, Philadelphia: Open University Press.

Crumlish, C. M. (1994). Coping and emotional response in cardiac surgery patients. Western Journal of Nursing Research: a forum for communicating nursing research, $1611,57-68$

Cummings, S. R., Stein, M. J., Hansen, B., Richard, R. J., Gerbert, B. \& Coates, T. J. (1989). Smoking counseling and preventive medicine. A survey of internists in private practices, and a health maintenance organization. Archives of Internal Medicine, 1492 ). 345-9.

Curry, S. J., \& McBride, C. M. (1994). Relapse prevention for smoking cessation: review and evaluation of concepts and interventions. Annual Review of Public Health, 15(1), 345-66. 
Dalstra, 1.A.A., \&eitsma, J.B. (1997). Hart-en vadziekten in Nederland 1997, cijfers over ziekte en sterfte. /Cardiovascular diseases in the Netherlands in 1997, rates on morbidity and mortality. Den Haag: Nederlandse Hartstichting.

Damoiseaux, $V . \&$ Kok, G. J. (1993). De planning van gezondheidseducatieve interventies [The planning of health education interventions]. In V. Damoiseaux $x_{s} H$. T. van der Molen, \& G. J. Kok (Eds.), Gezondheidsvoorlichting en gedragsverandering /Health education and behavioral change/ (pp. 75-86). Assen: Van Gorcum.

De Vries, H. (1998). Planning and evaluating health promotion. In D. W. Scott, R. Weston (Eds). Evaluating Health Promotion (pp. 92-108). London: Stanly Thomes.

De Vries, H. \& Backbier, E. (1994). Self-eficacy as an important determinant of quitting among pregnant women who smoke: The 0 pattern. Preventive Medicine, 23(2), 167-174.

De Vries, H. Backbier, E, Kok, G. \& Dijkstra, M. (1995). The impact of social influences in the context of attitude, self-efficacy, intention and previous behaviour as predictors of smoking onset. Journal of Applied Social Psychology 25(3), 237-257.

De Vries, H., \& Brug. J. (1999). Computer-tailored interventions motivating people to adopt health promoting behaviours: introduction to a new approach [editorial] [In Process Citation]. Patient Education and Counseling, 36(2), 99-105.

De Vries, H., Dijkstra, M. \& Kuhliman, P. (1988). Self-efficacy: The third lactor besides attitude and subjective nom as a predictor of behavioural intentions. Health Education Research, 3(3), 273-282.

De Vries, H., \& Kok, G. (1996). Gezondheidsbewordering: een toepassing wan het $A B C$ planningsmodel [Health Promotion: an application of the $A B C$ planning model]. Gedrag en Gezondheid (24), 342-352.

De Vries, H., \& Mudde, A. N. (1998). Predicting stage transitions for smoking cessation applying the attitude-social influence-efficacy model. Psychology and Health, 13 , 369-385.

De Vries, H., Mudde, A. N., Dijkstra, A., \& Willemsen, M. C. (1998). Differential beliefs, perceived social influences, and self-efficacy expectations among smokers in various motivational phases. Preventive Medicine, 27, 681-9.

DeBusk, R. F., Miller, N. H., Superko, H. R., Dennis, C. A., Thomas, R. J. Lew, H. T., Berger, W. E., Heller, R. S., Rompf, J, Gee, D., et al. (1994). A case-management system for coronary risk factor modification after acute myocardial infarction [see comments]. Amals of Internal Medicine, 12099), $721 \mathrm{~m} 9$

Deckers J. W. \& Agema, W. R. P. (1994). Effecten van stoppen met roken van sigaretten biij patiënten met coronairlijden lEffects of smoking cessation in patients with coronary heart diseasel. Hart Bulletin 25, 3-8.

Deckers, J. W. Agema, W. R. P. Hujbrechts, I. P. A. M. Erdman, R. A. M., Boersma, H. \& Roelandt, J. R. T. C. (1994). Quilting smoking in patients, with recently established coranary artery disease reduces mortality by ower $40 \%$ : Results of a meta-analysis. European Heart Joumal, 171, 1008. 
Dekker; H. M. (1990). Roken in de gezondheidszorg [Smoking in health care]. Medist Contact, 45(43), 1283-1285.

DeMello, D. I., Hoffman, L. A. Wesmiller, S. W., \& Zullo, T. G. (1989). Smoking and attitudes toward smoking among clinical nurse specialists, critical care nurses, and me* dical-surgical nurses. Oncology Nursing Forum, 16(6), 795-9.

DeValle, M. N., \& Norman, P. (1992). Causal attributions, health locus of control beliefs and lifestyle changes among pre-operative coronary patients. Psychology and Health $7,201-211$.

DiClemente, C. C., Prochaska, .. O., Fairhurst, S. K., Velicer, W. F. Velasquez, M. M. \& Rossi, J. S. (1991). The process of smoking cessation: an analysis of precontemplation, contemplation, and preparation stages of change. Journal of Consulting and Clinical Psychology, 5942), 295-304.

Dignan, M. (1993). Power analysis: A useful tool for designing health education and health promotion research. Health Values, 17, 55-57.

Dijkstra, A., De Viries, H., \& Bakker, M. (1996). Pros and cons of quitting, self-efficacy, and the stages of change in smoking cessation. Jowmal of Consulting and Clinical P5ychology, 64(4), 758-763.

Dijkstra, A., De Vries, H., \& Roilackers, 1. (1998a). Long-term effectiveness of computergenerated tallored feedback in smoking cessation. Health Education Research, 13(2), 207-214.

Dijkstra, A., De Vries, H., Roijackers, J., \& Van Breukelen, G. (1998b), Tailored interwentions to communicate stage-matched information to smokers in different motivational stages. Journal of Consulting and Clinical Psychology, 66(3), 549-557.

Dijkstra, M. (1995). Development, implementation and evaluation of two Dutch smoking prevention programs. Maastricht:Universitaire Pers Maastricht.

Doerfler, L. A., Pbert, L., \& DeCosimo, D. (1994). Symptoms of posttraumatic stress disorder following myocardial infarction and coronary artery bypass surgery (see com. ments]. General Hospital Psychatry, 16(3). 193-9.

Doll, R., Peto, R., Wheatley, K., Gray, R., \& Sutherland, 1. (1994). Mortality in relation to smoking: 40 years' observationis on male British doctors [see comments]. British Medical journal, 3096959), 901-11.

Domburg, R. T. wan., Meeter, K., Van Berkel, T. F. M., Veldkamp, R. F. Herwerden, L. A. van, \& Bogers, A. J. J. C. (2000). Smoking cessation reduces mortality after coronary artery bypass surgery. Jounal of the American College of Cardiology, 36(3), 878-83.

Dore, K., \& Hoey, I. (1988). Smoking practices, knowledge and attitudes regarding smoking of university hospital nurses. Canadian Joumal of Public Heath, 793), 170-4.

Duistermaat, J. H. (1999). Eindverslag 'stoppen met roken' van tijdelijk project naar continu beleid /Final report 'smoking cessation' 1996-1999 from temporary project toward continue policy. Utrecht: Landelijke Huisartsen Vereniging. 
Duncan, C., Stein, M. $\|_{\&}$ \& Cummings, S. P. (1991). Staff involvement and special followup time increase physicians counseling about smoking cessation: A controlled trial. American Jounal of Public Health, 81(7), 899-901.

Dutch Foundation on Smoking and Health. (1999). Jaarverslag 1998. IAnnual report 19981. Den Haag: Stichting Volksgezondheid en Roken.

Eagly, A. H. \& Chaiken, 5. (1993). The psychology of attitudes. Fort Worth: Harcourt Brace College Publishers.

Emmons, K. M. Goldstein, M. G. (1992). Smokers who are hospitalized: a window of opportunity for cessation interventions. Preventive Medicine, 21(2), 262-9.

Erdman, R. A. M. Duiverwoorden, H. I., Verhage, F, Kazemier, M. \& Hugenholz, P. G. (1984). Hartrevalidatie: een vervolgonderzoek over 5 jaar psychisch functioneren, werkhervating, rookgewoonten en sportieve activiteiten ICardiac rehabilitation: A 5 year follow-study into psychological functioning, resumption of work, smoking haw bits and physical activities. Nedertands Tijdschrift voor Geneeskunde 128, 846-51.

Erica Research Group. (1991). Prediction of coronary heart disease in Europe. The 2nd report of the WHO-ERICA Project. ERICA Research Group. European Heart Jounal, 12(3), $291-7$

EUROASPIRE Study Group. (1997). A European Society of Cardiology survey of seconda" ry prevention of coronary heart disease: principal results. EUROASPIRE Study Group. European Action on Secondary Prevention through Intervention to Reduce Events [published erratum appears in European Heart Journal 1998 Feb;19(2): 356-7]. European Heart Journal, 18(10), 1569-82.

Eurostat. (1997). (Annual Yearbook 1997: A statistical look on Europe 1986-1996). Een statistische blik op Europa 1986-1996]. Luxembourg: Office for official publications of the Europuean communities.

Falger, P. R. J., Sebregts, E. H. W. L., Leuteren, K. S. J. van, \& Bär, F. W. H. M. (2000). Wederzijdse beïnvloeding var de kwaliteit wan leven van patiënten en significante anderen na een hartinfarct of een coronary bypass graft operatie llmpact of depressed mood on mutual quality of life in cardiac patients and partners after a coronary event). Gedrag \& Gezondheid, 2845), 274-287.

Faulkner, A. \& Ward, L. (1983). Nurses as health educators in relation to smoking. Nursing Times, $79(15), 47-8$.

Festinger L. (1976). A theory of cognitive dissonance. Stanford, Califomia: Stanford, U.P. Fiole, M. C. (1997). AHCPR smoking cessation guideline: a fundamental review. Tobacco Control, $6($ Suppl. H), S4-8.

Fiore, M. C. et al. (2000). Treating Tobacco Use and Dependence. Clinical Practice Guideline (AHRQ publication No. 00-0032). Rockville: U.S. Department of Health and Human Services.

Fiore, M. C., Jorenby, D. E., \& Baker, T. B. (1997). Smoking cessation: principles and practice based upon the AHCPR Guideline, 1996. Agency for Health Care Policy and Research. Annals of Behavioral Medicine, 19(3), 213-9. 
Fore, M. C., Novotny, T. E. Pierce, I. P. Giovino, G. A., Hatziandreu, E. J, Newcomb, P. A., Surawiz, T. S., \& Davis, R. M. (1990). Methods used to quit smoking in the United States. Do cessation programs help? (published erratum appears in Journal of the American Medical Association 1991, 16:265(3):358) [see comments]. Jourmal of the American Medical Association, 263(20), 2760-5.

Fishbein, M. \& Ajzen, 1. (1975). Belief, attitude, intention and behavior" an introduction to theory and research. Reading, MA: Addison-Wesley.

Flay, B. R., \& Petraitis, 1. (1994). The theory of triadic influence: a new theory of health behavior with implications for preventive interventions. Advances in Medica/ Sociology 4, 19-44.

Fowler, G. (1997). Smoking cessation: the role of general practitioners, nurses and pharmacists. In C. T. Bolliger \& K. O. Fagerstrom (Eds), The tobacco epidemic (pp. 16577). Basel: Karger.

Frank, E., \& Kunovich-Frieze, T. (1995). Physicians" prevention counseling behaviors: current status and future directions [editorial; comment]. Preventive Medicine, 246 , 543-5.

Frank, E., Winkieby, M. A., Altman, D. G., Rockhill, B., \& Fortmann, 5. P. (1991). Predictors of physician's smoking cessation advice [see comments]. Joumal of the American MedicalAssociation, 266(22), 3139-44.

Freund, K. M., D'Agostino, R. B., Belanger, A. J., Kannel, W. B., \& Stokes, J. (1992). Predictors of smoking cessation: the Framingham Study. American Journal of Epidemiology, 135(9), 957-64.

Frid, D., Ockene, I. 5., Ockene, J. K., Merriam, P., Goldberg, R., Kristeller, J. \& Barrett, 5. (1991). Severity of angiographically proven coronary artery disease predicts smoking cessation. American Jounal of Preventive Medicine, 7(3), 131-5.

Gerards, F. (1993). Health Counseling. In V. Damoiseaux, H. T. van der Molen, \& G. J. Kok (Eds.), Gezondheidsvoorlichting en gedragsverandering / health education and behavioral change) (pp. 353-361). Assen: Van Gorcum.

Gerards, F. (1997). Health Counseling Baarn: H. Nelissen.

Gerards, F. M. \& Hospers, H. (1991). Health Counselling: een strategie voor het individueel begeleiden van adviezen (Health counseling: a strategy to accompany patient guidance'. Nederlands Tyidsctrift voor Dietisten, 46(5), 132-137.

Glasgow, R. E. Muillooly, J. P., Vogt, T. M., Stevens, V. I., Lichtenstein, E., Hollis, I.F, Landa, H.A. Severson, H.H., Pearson, K.A., Vogt, M.R. (1993). Biochemical validation of smoking 5tatus: Pros, cons, and data from four low-intensity intervention trials. Addictive Behaviors, $18(5), 511-527$.

Glasgow, R. E., Stevens, VI., Vogt, T.M. Mullooly, L.P., Lichtenstein, E. 11991). Changes in smoking associated with hospitalization: Quit rates, predictive variables, and intervention implications. American Journal of Health Promotion, 6(1), 24-29. 
Glynn, T. J., Boyd, G. M. \& Gruman, J. C. (1990). Essential elements of self-helpiminimal intervention strategies for smoking cessation. Health Education Quarterly, 17(3), $329-45$

Godin, G. \& Kok, G. (1996). The theory of planned behavior: a review of its applications to health-related behaviors. American Journal of Health Promotion, $11(2), 87-98$.

Goldstein, A. O., Hellier, A., Fitzgerald, S., Stegall, T. S., \& Fischer, P. M. (1987). Hospital nurse counseling of patients who smoke. American Journal of Public Health, 77,10), 1333-4.

Goldstein, H. (1995a). Hierarchical data modeling in the social sciences. Joumal of Educational and Behavioral Statistics, 2022), 201-204.

Goldstein, H. (1995b). Multilevel Statistical Madel. London: Edward Amold.

Goodman, M. J., Nadkarni, M. \& Schorling, J. B. (1998). The natural history of smoking cessation among medical patients in a smoke-free hospital. Substance Abuse, 19(2), 71-79.

Goodman, R. M., \& Steckler, A. (1989). A model for the institutionalization of health promotion programs. Family and Community Health,(Februari), 63-78.

Gourlay, S. G. \& Benowitz, N. L. (1996). The benefits of stopping smoking and the role of nicotine replacement therapy in older patients. Drugs Aging, 9(1), 8-23.

Green, L. W. \& Kreuter, M. W. (1991). Health Promotion Planning: An Educational and Environmental Approach (2th ed.). Mountain View: Mayfield Publishing Company.

Green, L. W. \& Kreuter, M. W. (1999). Health Promotion Planning: An Educational and Ecological approach (3th ed.). California: Mayfield publishing company.

Greenwood, D. C., Muir, K. R., Packham, C. J., \& Madeley, R. J. (1995). Stress, social support, and stopping smoking after myocardial infarction in England. Journal of Epidemiology and Community Health, 49(6), 583-7.

Gupta, R., Gupta, K. D., Sharma, S., \& Gupta, V. P. (1993). Influence of cessation of smoking on long term mortality in patients with coronary heart disease. Indian Heart Journal, 45(2), 125-9.

Hajek, P. (1994). Treatments for smokers. Addiction, 89111), 1543-1549.

Hansen, W. B., Malotte, C. K., \& Fielding, J. E. (1985). The bogus pipeline revised: the use of the threat of detection as a means of increasing self-reports of tobacco use. Journal of Applied Psychology, 70(4), 789-92.

Haustein, K. O. (1999). Smoking, cardiovascular diseases and possibilities for treating nicotine dependence. Wiener Medische Wochenschrift, 149(1), 19-24.

Havik, O. E, \& Maeland, J. G. (1988). Changes in smoking behavior after a myocardial infarction. Health Psychology, 7(5), 403-20.

Havik, O. E., \& Maeland, J. G. (1990). Patterns of emotional reactions after a myocardial infarction. Jounnal of Psychosomatic Research, 34(3), $271-85$.

Hayford, K. E., Patten, C. A.s Rummans, T. A.s Schroeder, D. R., offord, K. P., Croghan, I. T., Glover, E. D., Sachs, D. P. L., Hurt, R. D. (1999). Efficacy of bupropion for smoking cessation in smokers with a former history of major depression or alcoholism. British Journal of Psychiatry 174, 173-8. 
Hays, S. L. (1981). Statistics. New York: Holt Rinehart \& Winston.

Heatherton, T. F., Kozlowski, L. T., Frecker, R. C., \&agerstrom, K. O. (1991). The Fagerstrom Test for Nicotine Dependence: A revision of the Fagerstrom Tolerance Questionnaire. British Joumal of Addiction, 86(9), $1119-1127$.

Hemingway, H., \& Marmot, M. (1999). Evidence based cardiology: psychosocial factors in the aetiology and prognosis of coronany heart disease. Systematic review of prospective cohort studies. British Medical Joumal, 318(7196), 1460-7.

Hollis, J. F., Lichtenstein, E., Mount, K., Vogt, T. M. \& Stevens, V. L. (1991). Nurse-assisted smoking counseling in medical settings: minimizing demands on physicians. Preventive Medicine, 2044), 497-507.

Huijbrechts, I. P., Duivenvoorden, H. I., Deckers, H. W. Leenders, I. C., Pop, G. A. Passchier,

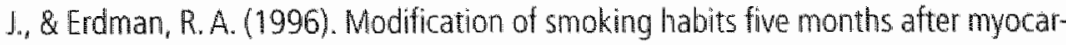
dial infarction: relationship with personality characteristics. Jounal of Psychosomatic Research, 40(4), 369-78.

Irvin, \. E., Bowers, C. A., Dunn, M. E. \& Wang, M. C. (1999). Efficacy of relapse prevention: a meta-analytic review. Joumal of Consulting and Chical Psychology, 67(4), 563-70.

Jaarsma, T., Kastermans, M., Dassen, T., \& Philipsen, H. (1995). Problems of cardiac patients in early recovery. Joumal of Advanced Nursing, 21(1), $21-7$.

Jansen, 1. (1994). Coronary heart diseases and cerebrovascular diseases in the Netherlands (publication no. 442003004). Bilthoven: National Institute of Public Health and the Environment [RIVM].

Johnson, J. L., Budz, B., Mackay, M., \& Miller, C. (1999). Evaluation of a nurse-delivered smoking cessation intervention for hospitalized patients with cardiac disease. Heart and Lung, 28(1), 55-64.

Jorenby, D. E., Leischow, S. J., Nides, M. A., Rennard, S. I., Johnston, J. A., Hughes, A. R., Smith, S. 5. Muramoto, M. L., Daughton, D. M., Doan, K., Fiore, M. C., Baker, T. B. (1999). A controlled trial of sustained release bupropion, a nicotine patch, or both for smoking cessation [see comments]. New England Journal of Medicine, 34099). $685-91$.

Kiesler, C. A. (1971). The psychology of commitment. New York: Academic Press.

Kirk, R. E. (1982). Expermental design procedures for the behavioral sciences (2th ed.). Pacific grove California: Brooks/Cole publishing company.

Kok, G., de Vries, H., Mudde, A. N., \& Strecher, V. J. (1991). Planned health education and the role of self-efficacy: Dutch research. Health Education Research, 6(2), 231-238.

Kok, G. Van den Borne, B., \& Mullen, P. D. (1997). Effectiveness of health education and health promotion: meta-analyses of effect studies and determinants of effectiveness. Patient Education and Counseling, 3011), 19-27.

Konings-Dalstra, J.A.A., Reitsma, J. B. (1999). Hart en vatziekten in Nederland 1999. ciffers over ziekte en sterfte /Cardiovascular diseases in the Netherlands in 1999 rates on morbidity \& mortality?. Den Haag. Nederlandse Hartstichting. 
Kottke, T. E. Battista, R. N. Defriese, G. H. \& Brekke, M. L. (1988). Attrbutes of successful smoking cessation interventions in medical practice. A meta-analysis of 39 controlled trials. Journal of the American Medical Association, 259(19), 2883-9. Kristeller, J. L. Rossil, J. S. Ockene, J. K., Goldberg, R., \& et al. (1992). Processes of change in smoking cessation: A cross-validation study in cardiac patients. Joumal of Substance Abuse, 4(3), $263-276$.

Kviz, F. J. Clark, M. A., Prochaska, T. R. Slezak, J. A, Crittenden, K. S., Freels, S. \& Camplbell, R.T. (1995). Attitudes and practices for smoking cessation counseling by provider type and patient age. Preventive Medicine, 24(2), 201-12.

Lancaster, T., Silagy, C., Fowler G. \& Spiers, 1. (1999). Training health professionais in smoking cessation (Cochrane Review). The Cochrane Library, issue 4. Oxford: Update Software.

Lando, H. A. (1993). Formal quit smoking treatments. In C. T. Orleans \& 1. Slade (Eds.). Nicotine addiction: Principles and management (pp. 221-224). New York: Oxford University Press.

Lando, H. A., Hellerstedt, W. L. Pirie, P. L., \& McGovern, P. G. (1992). Brief supportive telephone outreach as a recruitment and intervention strategy for smoking cessation. American Joumal of Public Health, 82(1), 41-46.

Latter, S., Clark, J. M., Wilson-Barnett, J., \& Maben, J. (1992). Health education in nursing: perceptions of practice in acute settings. Journal of Advanced Nursing, $17(2)$, $164-72$

Lechner, L. (1998). Social psychological determinants of health risk behawors related to cancer and CVD. [PhD thesis]. Maastricht: Universitaine Pers Maastricht.

Lechner, L., Brug. J., De Vries, H., Van Assema, P., \& Mudde, A. (1998). Stages of change for fruit, vegetable and fat intake: consequences of misconception. Health Education Research, 13(1), 1-11.

Leest, L. A. T. M. van, Hulscher, M. E. J. L., Tsang, S, Schattenberg, G., \& Grol, R. P. T. M. (1999). Interim-rapport evaluatie van hel LHVNHG project 'Preventie: maatwerk, hart-en vaatziekten'1998-1999 /interim report evaluation of the $\angle H V / N H G$ project Prevention: tailor-made; cardiovascular diseasel. Nimegen: Katholieke Universiteit Nijmegen.

Lichteristein, E. \& Glasgow R. E. (1992). Smaking cessation: What have we learned over the past decade? Journal of Consulting and Clinical Psychology, 6044), 518 527.

Lichtenstein, E. Glasgow, R. E. Lando, H. A. Ossip-Klein, D. J. \& Boles, S. M. (1996). Telephone counseling for smoking cessation: rationales and meta-analytic review of evidence. Health Education Research, $11(2), 243-57$.

Lillington, L. M. (1997). AHCPR smoking cessation guideline goals and impact: exampw les from the nursing field. Tobacco Control, 6(Suppl. 1), 539-43. 
Little, R. J. A. \& Yau, L. H. Y. (1998). Statistical techniques for analyzing data from prevention trials: treatment of no-shows using Rubin's causal modiel. Psychological Methods, 3, 147-159.

Locke, E. A., \& Latham, G. P. (1990). A theory of goal setting and task performance. Englewood Cliffs: Prentice Hall.

Locke, E. A., \& Latham, G. P. (1994), Goal setting theory. In H. F. O'Neil, \& M. Drillings (Eds.), Motwation: Theory and research. (pp. 13-29). Hillsdale, USA: Lawrence Erlbaum Associates, inc.

Longtord, N. T. (1995). Random Coefficient Models. Oxford: Clarendon Press.

MacDonald, G. Veen, C. \& Tones K. (1996). Evidence for success in health promotion: suggestions for improvement. Health Education Research, 1/(3), 367-76.

MacDonald, V., \& Muir, J. (1996). Implementing innovations in health care settings. Canadian Nurse, $92(9), 31.3$.

Maibach, E. \& Parrott, R. L. (1995). Designing health messages. Thousand oaks: Sage. Malotte, C. K., Jarvis, B., \& Fishbein, M. (2000). Stage of change versus an integrated psychosocial theory as a basis for developing effective behaviour change interventions. AIDS Care, 12(3), 357-364.

Marlatt, G. A., \& Gordon, J. R. (1985). Relapse prevention: Maintenance strategies in the treatment of addictive behaviors. New York: Guildford.

Marshall, P. (1990). "Just one more. . "' A study into the smoking attitudes and behavior of patients following first myocardial infarction. International Jounal of Nursing Studies, 27(4), 375-87.

McCauley, K. M. (1995). Assessing social support in patients with cardiac disease. Journal of Cardiovascular Nursing, 10(1), 73-80.

McGinnis, J. M., \& Foege, W. H. (1993). Actual causes of death in the United States. Journal of the American Medical Association, 270(18), 2207-2212.

MCGuire, W. J. (1985). Attitudes and attitude change. In G. Lindsey \& E. Aronson (Eds.), The handbook of social psychology (pp. 233-346). New York: Random House.

Mckenna, K., \& Higgens, H. (1997). Factors influencing smoking cessation in patients with coronary artery disease. Patien Education and Counseling, 32, 197-205.

Mehta, R. H. \& Eagle, K.A. (1998). Secondary prevention in acute myocardial infarction [see comments]. British Medical Jownal, 3167134), 838-42.

Mesters, I., Creers, T. L., \& Gerands, F. (in press). Self-Management and Respiratory Disorders: Guiding Patients from Health Counseling and Self-Management Perspectives. In A. Kaptein \& T. Creer (Eds.). Respiratory disorders and behavioral research. Harwood Academic Publishers.

Mesters, I., \& Meertens, R. M. (1999). Monitoring the dissemination of an educational protocol on pediatric astma in family practice: A test of associations between dissemination variables. Health Education and Behavior, 26(1), $103-120$.

Miller, N. H. (1993). Tips for smoking cessation. Heart Disease and Stroke, 2(1), 5-7. 
Miler, N. H., Smith, P. M., DeBusk, R. F. Sobel, D. S., \& Taylor, C. B. (1997). Smoking cessation in hospitalized patients. Results of a randomized trial. Archives of internal Medicine, 157(4), 409-15.

Miller, W. R., \& Rollnick, S. (1991). Motivational interviewing: Preparing people to change addictive behavior. New York, USA: Guilford Press.

Miwa, K., Fujita, M., Miyagi, Y., Inoue, H. \& Sasayama, S. (1995). Is smoking behavior in patients with coronary heart disease influenced by whether their attending physician smokes. Tobacco Control, 4, 236-238.

Moser, D. K. \& Dracup, K. (1995). Psychosocial recovery from a cardiac event: the influence of perceived control. Heart and Lung, 24(4), 273-80.

Moser, D. K., Dracup, K. A., \& Marsden, C. (1993). Needs of recovering cardiac patients and their spouses: compared views. International Jounal of Nursing Studies, 30(2), 105-14.

Mudde, A. N "De Vries, H. \& Dolders, M. G. (1995a). Evaluation of a Dutch communitybased smoking cessation intervention. Preventive Medicine, 24(1), 61-70.

Muddle, A. N., De Wries, H., \& Strecher, V. J. (1996). Cost-effectiveness of smoking cessation modalities: comparing apples with oranges? Preventive Medicine, 25(6), 70816.

Mudde, A. N., Kok, G., \& Strecher, V. J. (1995b). Self-efficacy as a predictor for the cessa* tion of smoking: Methodological issues and implications for smoking cessation programs. Psychology and Health, 10(5), 353-367.

Mudde, A. N., Willemsen, M. C. \& Van Assema, P. (1994). Development and utilization of a self-help manual for community smoking cessation interventions. In $R$. Richmond (Ed.) interventions for Smokers: An International Perspective. Baltimore: Williams \& Wilkins.

Mulcahy, R. (1983). Influence of cigarette smoking on morbidity and mortality after myocardial infarction. British Heart Journal, 49(5), 410-5.

Mullen, P. D., Mains, D. A. \& Velez, R. (1992). A meta-analysis of controlled trials of cardiac patient education. Patient Education and Counseling, 192), 143-62.

Murray, C. J. L., \& Lopez, A. D. (1996). The global burden of disease. Geneva: The Harvard School of Public Health.

Murray, D. M. Hannan, P. J, \& Zucker, D. M. (1989). Analysis issues in school-based health promotion studies. Health Education Quarterly, 96(2), 315-20.

Murray, R. P., Connett, 1. E. Lauger, G. G. \& Voelker, H. T. (1993). Error in smoking measures: effects of intervention on relations of cotinine and carbon monoxide to self-reported smoking. The Lung Health Study Research Group. American Jounal of Public Health, 83(9), 1251-7.

Nagle, A., Schofield, M., \& Redman, S. (1999). Australian nurses' smoking behaviour, knowlegde and attitude towards prowiding smoking cessation care to their patients. Health Promotion International, 14(2), $133-144$. 
Nash, R., Edwards, H. \& Nebauer, M. (1993). Effect of attitudes, subjective norms and perceived control on nurses intention to assess patients' pain. Jounal of Advanced Nursing, $1846,941-7$.

NIPO. (1995). Enquete naar rookgewoonten van specialisten en verpleegkundigen INIPO-survey aimed at the smoking habits of medical doctors and nurses]. Den Haag: Stichting Volksgezondheid en Roken.

Norman, P. Conner, M. \& Bell, R. (1999). The theory of planned behavior and smoking cessation. Health Psychology, 18(1), 89-94.

Nutbeam, D. (1998). Evaluating health promotion-progress, problems and solutions. Health Promotion International, 13(1), 27-44.

Nutbeam, D. (1999). Evaluating health promotion [In Process Citation]. British Medical Journal, $318(7180), 404 \mathrm{~A}$.

Ockene, J., Kristeller, J. L., Goldberg, R. Ockene, 1., Merriam, P., Barrett, S. (1992). Smoking cessation and severity of disease: The Coronary Artery Smoking Intervention Study. Health Psychology, 11(2), 119-126.

Ockene, J. K., Emmons, K. M., Mermelsteim, R. J., Perkins, K. A., Bonollo, D. S., Voorhees, C. C.\& Hollis, J.F. (2000). Relapse and maintenance issues for smoking cessation. Health Psychology, 19suppl. 1), 17-31.

Ockene, J. K., Hosmer, D. Rippe, J., Williams, J., Goldberg, R. I., DeCosimo, D., Maher, P. M. \& Dalen, J. E. (1985). Factors affecting cigarette smoking status in patients with ischemic heart disease. Journal of Chronic Diseases, 38(12), 985-94.

Oldenburg, B., Hardcastle, D. M., \& Kok, G. (1997). Diffusion of Innovations, In K. Glanz, F. M. Lewis, \& B. K. Rimer (Eds.), Health Behavior and Health Education: Theory, Research and Practice (2nd ed, pp. 270-286). San Francisca: Jossey-Bass.

Oldenburg, B. F., Sallis, J. F., French, M. L., \& Owen, N. (1999). Health promotion research and the diffusion and institutionalization of interventions. Health Education Research, 14(1), 121-130.

Orlandi, M. A. (1987). Promoting health and preventing disease in health care setting:s: an analysis of barriers. Preventive Medicine, $16(1), 119-30$.

Orlandi, M. A., Landers, C. Weston, R. \& Haley, M. (1990). Diffusion of Heath Promotion Innovations. In K. Glanz, F. M. Lewis, \& B. K. Rimer (Eds.), Health Behavior and Health Education (pp. 289-313). San Francisco: Jossey-Bass Publishers.

Orleans, C. T., Glynn, T. J., Manley, M. W., \& Slade, J. D. (1993a). Mimimal-contact quit smoking strategies for medical settings. In C. T. Orleans \& J. D. Slade (Eds.), Nicotine addiction: Principles and management. (pp. 181-220). New York: Oxford University Press.

Orleans, C. T., Kristeler, J. L. \& Gritz, E. R. (1993b). Helping hospitalized smokers quit: New directions for treatment and research. Joumal of Consulting and Clinical Psychology, $67(5), 778-789$. 
Orleans, C. T, \& Ockene, I. K. (1993). Routine hospital-based quit-smoking treatment for the postmyocardial infarction patient: an idea whose time has come leditorial; comment]. Jounal of the American College of Cardiology, 22(6), 1703-5.

Orleans, C. T, Rotberg, H. L., Quade, D. \& Lees, P. (1990). A hospital quit-smoking consult service. clinical report and intervention guidelines. Preventive Medicine, 192), 198.212 .

Parcel, G, S., O'Hara-Tompkins, N. M., Harrist, R. B., Basen-Engquist, K. M., McCormick, L. K. Gottlieb, N. H. \& Eriksen, M.P. (1995). Diffusion of an effective tobacco prevention program. Part II: Evaluation of the adoption phase. Health Education Research, 10(3), 297-307.

Paulussen, T., Kok, G., \& Schaalma, H. (1994). Antecedents to adoption of classroombased AIDS education in secondary schools. Health Education Research, 94), 485 . 496.

Paulussen, T. Kok, G., Schaalma, H. \& Parcel, G. S. (1995). Diffusion of AlDS curricula among Dutch secondary school teachers. Health Education Quarterly, 22(2), 227-43.

Pearson, T., Rapaport, E., Criqui, M., Furberg, C., Fuster, W., Hiratzka, L., Little, W., Ockene, 1. \& Williams, G. (1994). Optimal risk factor management in the patient after coronary revascularization. A statement for healthcare professionals from an American Heart Association Writing Group. Circulation, 9066), 3125-33.

Perkins, K. A. (1988). Maintaining smoking abstinence after myocardial infarction. Journal of Substance Abuse, $1(1), 91 \% 107$.

Peto, R., Lopez, A. D., Borham, J., Thun, M., \& Heath, C. (1994). Mortality from smoking in developed countries: 1950-2000: indrect estimates from national vital statistics. New Vork: Oxford University Press.

Pieterse, M. E. (1999). Stoppen met roken met hulp van de huisartspraktijk (5moking cessation with help from the general practice]. [PhD thesis]. Enschede: Twente University Press.

Pieterse, M. E., Seydel, E. R., Mudde, A. N., \& De Vries, H. (1994). Uitwoerbaarheid en effectiviteit van een minimaal stoppen-met-roken programma voor de huisartsenpraktijk [Feasibility and efficacy of a minimal contad smoking cessation programme in the general practice]. Tijdschrift voor Gezondheidsbevordering, 15(2), 57-71.

Pomerleau, C. S., Carton, 5. M., Lutzke M. L. Flessland, K. A., \& Pomerleau, O. F. (1994). Reliability of the Fagerstrom Tolerance Questionmaire and the Fagerstrom Test for Nicotine Dependence. Addictive Behaviors, 1911), 33-9.

Post, D., \& Stokx, L. J. (1997). Zorgbehoeften en zorggebruik. (Health services needs and actual use]. In D. Ruwaard \& P. G. N. Kramers (Eds.), Volksgezondheid Toekornst Verkenning 1997. Public health status and forecasts 1997) (part 6). Den Haag: Elsevier/De Tijdstroom.

Prochaska, J. 0. (1991). Assessing how people change. Cancer, 67(3 Suppl), 805-7.

Prochaska, J. 0. (1996). A stage paradigm for integrating clinical and public health approaches to smoking cessation. Addictive Behaviors, 21(6), 721-32. 
Prochaska, J. O., \& DiClemente, C. C. (1983). Stages and processes of self-change of smoking: toward an integrative model of change. Journal of Consulting and Clinical Psychology, 51(3), 390-5.

Prochaska, J. O., \& Diclemente, C. C. (1992). Stages of change in the modification of problem behaviors. Progressions in Behavioural Modification, 28, 183-218.

Prochaska, J. O., DiClemente, C. C., \& Norcross, J. C. (1992). In search of how people change: Applications to addictive behaviors. American Psychologist, 479), 1102 1114.

Prochaska, J. O., Diclemente, C. C., Velicer, W. F., Ginpil, S., \& Norcross, J. C. (1985). Predicting change in smoking status for self-changers. Addictive Behaviors(10), 395-406.

Prochaska, J. O., \& Goldstein, M. G. (1991). Process of smaking cessation. Implications for clinicians. Clinics in Chest Medicine, 12(4), 727-35.

Prochaska, J. O., Norcross, J. C., \& DiClemente, C. C. (1994a). Changing for good. New York: William Morrow and Company, Inc.

Prochaska, J. O., \& Velicer, W. F. (1997). The transtheoretical model of health behavior change. American Journal of Health Promotion, 12(1), 38-48.

Prochaska, J. O, Velicer, W. F., Rossi, J. S., Goldstein, M. G., Marcus, B. H., Rakowski, W. Fiore, C., Harlow, L. L., Redding, C. A., Rosenbloom, D., et al. (1994b). Stages of change and decisional balance for 12 problem behaviors. Health Psychology. 13(1), 39-46.

Raw, M., McNeill, A., \& West, R. (1998). Smoking cessation guidelines for health professionals. A guide to effective smoking cessation interventions for the health care system. Health Education Authority. Thorax, 53(5uppl. 5), 51-19.

Raw, M., McNeill, A, \& West, R. (1999). Smoking cessation: evidence based recommendations for the healthcare system [see comments]. British Madical youmal, 31877177), 182-5

Reitsma, J. B. (1995). Hart- en vaatziekten in Nederland 1995, ciffers over ziekte en sterfte /Cardiovascular diseases in the Netherlands, rates on morbidity \& montalityl. Den Haag: Nederlandse Hartstichting.

Rice, V. H., Fox, D. H., Lepczyk, M., Sieggreen, M., Mullin, M., Jarosz, P., \& Templin, T. (1994). A comparison of nursing interventions for smoking cessation in adults with cardiovascular health problems. Heart and Lung, 23(6), 473-86

Rice, V. H. \& Stead, L.F. (1999). Nursing interventions for smoking cessation (Cochrane Review). The Cochrane Library, (issue 4), 1-14. Oxford: Update Sofinare.

Rice, V. H., Templin, T., Fox, D. H., Jarosz, P., Mullin, M., Seiggreen, M., \& Lepczek, M. (1996). Social context variables as predictors of smoking cessation. Tabacco Control(5), 280-5.

Rigotti, N. A., Arnsten, J.H., McKool, K. M., Wood-Reid, K. M., Pasternak, R. C., \& Singer, D. E. (1997). Efficacy of a smoking cessation program for hospital patients. Archives of InternalMedicine, 157(22), 2653-60. 
Rigotti, N.A. Mckool, K. M. \& Stiffman, S. (1994). Predictors of smoking cessation after cotonary artery bypass grat surgery. Results of a randomized trial with 5-year follow-up. Annals of internal Medicine, 12044), $287-93$.

Rigotti, N. A. Singer, D. E. Mulley, A. G., Jr. \& Thibault, G. E. (1991). Smoking cessation following admission to a coronary care unit. Jounal of General Internal Medicine, 64). $305-11$.

Robins, I. M. \& Greenland, S. (1994). Adjusting for differential rates of prophylaxis therapy for PCP in high-versus low-dose AZT treatment amms in an AlDS randomized trial. Journal of the American Statistical Association, 89(427).737-748.

Robinson, L. D. \& Jewell, N. P. (1991). Some surprising results about covariate adjustment in logistic regression. International Statistical Review, 58, 227-240.

Rogers, E. M. (1995). Diffusion of Innovations (4th ed.), New York: The Free Press.

Rosal, M. C., Ockene, J. K., Ma, Y., Hebert, J. R., Ockene, I. 5., Merriam, P., \& Hurley, T. G. (1998). Coronary artery smoking intervention study (CASIS): 5-Year follow-up. Health Psychology, 17(5), 476-478.

Rose, $G_{2}$ \& Colwell, L. (1992) Randomised controlled trial of anti-smoking advice: Final (20 year) results. Joumal of Epideniology and Community Health, 46(1), 75-77.

Rossi, P. H., \& Freman, H. E (1993). Evaluation: A systematic approach (5th ed.). Newbury Park: Sage.

Ruwaard, D, \& Kramers, P. G. N. (1997). Volksgezondheid Toekomst Verkenning 1997: De som der delen (Public health status and forecasts 1997: Summing up the different parts]. Den Haag: Elsevier/De Tijdstroom.

Sackett, D. L., Haynes, R. B. Guyatt, G. H., \& Tugwell, P. (1991). Clinical epidemiology" a basic science for dinical medicine (2th ed.). Boston: Little, Brown and Company.

Sanders, D. J., Stone, V., Fowler, G., \& Marzillier, J. (1986). Practice nurses and antismoking education. British Medical Journal, 292(6517), 381-3.

Sandvik, L., Erikssen, J., Thaulow, E., Erikssen, G., Mundal, R., \& Rodahl, K. (1993). Physical fitness as a predictor of mortality among healthy, middle-aged Norwegian men [see comments]. New England Joumal of Medicine, 328(8), 533-7.

Sarafino, E. P. (1998). Health psychology: biopsychosocial interactions (3th ed.). New York: John Wiley \& Sons, inc.

Schneiderman, N. \& Skyler, J. S. (1996). Insulin Metabolism, Sympathetic Nervous System Regulation, and Coronary Heart Disease. In K. Orth-Gomer \& N. Schneiderman (Eds), Behavioral Medicine Approaches to Cardiovascular Disease Prevention (pp. 105-134). Mahwah, New Jersey: Lawrence Erlbaum Associates.

Scott, R. R., Mayer, J. A., Denier, C. A., Dawson, B. L., \& Lamparski, D. (1990). Long-term smoking status of cardiac patients following symptom-specific cessation advice. Addictive Behaviors, 15(6), 549-52.

Shaten, B. J., Kuller, L. H., \& Neaton, J. D. (1991). Association between baseline risk factors, cigarette smoking, and CHD mortality after 10.5 years. MRFIT Research Group. Preventive Medicine, 20(5), 655-9. 
Shaw, P. J., Bates, D., Cartidge, N. E. French, J. M., Heaviside, D., Julian, D. G, \& Shaw, D. A. (1987). Neurologic and netropsychological morbidity following major surgery: comparison of coronary artery bypass and peripheral wascular surgery. Stroke. 18(4), $700-7$

Shiffman, 5. (1982). Relapse following smoking cessation; a situational analysis. Journal of Consuting and Cinical Psychology, 50(1), $71-86$.

Simon, J.A., Browner, W. S. \& Mangano, D. T. (1992). Predictors of smoking relapse after noncardiac surgery. Study of Perioperative ischemia (SPI) Research Group. American Journal of Public Health, 82(9), 1235-7.

Sirota, A. D., Curran, J. P. \& Habif, V. (1985). Smoking cessation in chronically ill medical patients. Jounal of Clinical Psychology, 41(4), 575-9.

Smith, S. C. (1997). The challenge of risk reduction therapy for cardiovascular disease [see comments]. American Family Physician, 55(2), 491-500.

Smith, S. C, Jr, Blair, S. N. Criqui, M. H., Fletcher, G. F. Fuster, V., Gersh, B. J., Gotto, A. M., Gould, K. L., Greenland, P., Gundy, S. M. et al. (1995). Preventing heart attack and death in patients with coronary disease. Circulation, 92(1), 2-4.

Snow, M. G., Prochaska, J. 0. \& Rossi, J. S. (1992). Stages of change for smoking cessation among former problem drinkers. Journal of Substance Abuse(4), 107-116.

Solymoss, B. C., Nadeau, P. Millette, D., \& Campeau, L. (1988). Late thrombosis of saphenous vein coronary bypass grafts related to risk factors. Circulation, 78(Part 2), 1140-3.

Sparrow, D., \& Dawber, T. R. (1978). The influence of cigarette smoking on prognosis after a first myocardial infarction. A report from the Framingham study. Journal of Chronic Diseases, 37(6-7), 425-32.

Statistics and Epidemiology Research Corporation. (1993). Egret Reference Manual. Seattle/Washington.

Statistics Netherlands. (1997). Vademecum health statistics. Den Haag: SDU.

Steckler, A., \& Goodman. R. M. (1989). How to institutionalize health promotion programs. American Journal of Health Promotion, 3(4), $35-44$.

Steckler, A. Goodman, R. M., Mcleroy, K. R., Davis, S., \& Koch, G. (1992). Measuing the diffusion of innowative health promotion programs, American sournal of Health Promotion, 6(3). 214-24

Stein, D., Troudant, T. Hymowitz, Z, Gotsman, M., \& Kaplan De-Nour, A. (1990). Psychosocial adjustment before and after coronary artery bypass surgery. Intemational Jounal of Psychatry in Medicine, 2012), $181-92$.

Stevens, V. J., Glasgow, R. E., Hollis, J.F., Lichtenstein, E, \& Vogt, T. M. (1993). A smokingcessation intervention for hospital patients. Medical Care, 3N(1), 65-72

Strecher, V. J., Becker, M. H. Kirscht, J. F., Eraker, S. A., \& Graham-Tomasi, R. P. (1985). Evaluation of a minimal-contact smoking cessation program in a health care setting. Patient Education and Counseling, 7(4), 395-407.

Strecher, V. J., Devellis, B. M., Becker, M. H. \& Rosenstock, I. M. (1986). The role of selfefficacy in achieving health behavior change. Health Education Quarterly, $13(1), 73-92$. 
Strecher, V. L. Rimer, B. K. \& Monaco, K. D. (1989). Development of a new self help guide -Freedom From smoking for you and your family. Health Education Quarterly, 1611), $101-12$.

Sussman, 5., Dent, C. W. Stacy, A. W., Sun, P. el al. (1993). Project Towards No Tobacco Use: 1-year behavior outcomes. American Joumal of Public Health, 83(9), 12451250.

Tabachnick, B. G., \& Fidell, L. S. (2001). Using Multwariate Statistics (4th ed.). Boston: Allyn and Bacon.

Taylor, C. B., Houston Miller, N., Killen, J. D. \& DeBusk, R. F. (1990). Smoking cessation after acute myocardial infarction: effects of a nurse-managed intervention. Annals of internal Medicine, 113(2), 118-23.

Taylor, C. B., Miller, N. H., Herman, S., Smith, P. M., Sobel, D., Fisher, L., Debrusk, R. F. (1996). A nurse-managed smoking cessation program for hospitalized smokers. American Joumal of Public Health, 86(11), 1557-1560.

Tessier, J. F., Thomas, D., Nejjari, C, Belougne, D." \& Freour, P. (1995). Attitudes and opinions of French cardiologists towards smoking. European Journal of Epidemiology. $11(6), 615-20$.

Theobald, K. (1997). The experience of spouses whose partmers have suffered a myocardial infarction: a phenomenological study. Journal of Advanced Nursing, 26.3\%, 595601.

Tones, K. (1997). Beyond the randomized controlled trial: a case for 'judicial review' leditorial]. Health Education Research, 12(2), i-iw.

Tones, $K$, \& Tilford, 5. (1994). Health Education: Effectiveness, efficiency and equity (2th ed.). London: Chapman \& Hall.

Tonstad, S. (1999). Nicotine preparations and other preparations for smoking cessation. Tiósskr Nor Laegeforen, 1199), 1289-93.

Townes, B. D., Bashein, G., Hombein, T. F., Coppel, D. B., Goldstein, D. E., Davis, K. B., Nessly. M. L. Bledsoe, S. W. Veith, R. C., Ivey, T. D., et al. (1989). Neurobehavioral outcomes in cardiac operations. A prospective controlled study. Jounal of Thorac Cardiovascular Surgery, 98(5 Part 1), 774-82.

U.S. Department of Heath and Human Services. (1983). The Health Consequences of Smoking: Cardiovascular Disease. A report of the Surgeon General's office on 5 moking \& Health (DHHS Publication No. 84-50204). Washington, DC: U.S. Government Printing Office.

U.S. Department of Health and Human Services. (1990). The Health Benefits from 5 moking Cessation. A report of the Surgeon General's office on smaking \& health. (DHHS Publication No. (CDC) 90-8416). Washington, DC: U.S. Govemment Printing Office.

U.S. Department of Health and Human Services. (1996). Smoking Cessation: Clinical Practice Guidelines (DHHS Publication No. (AHCPR) 96-0892). Washington, DC. U.S. Government Printing Office. 
Van Assema, P., Brug, 1., Glanz, K., Dolders, M., \& Mudde, A. (1998). Nationwide implementation of guided supermarket tours in The Netherlands: a dissemination study. Health Education Research, 13(4), 557-66.

Van Berkel, T. F. M. Vlugt, M. J. van der, \& Boersma, H. (2000). Characteristics of smokers and long-term changes in smoking behaviour in consecutive patients with a myocardial infaction. Preventive Medicine, 31(6), 732-41.

Van Berkel, T. F. M., Boersma, H., De Baquer, D., Deckers, J. W., \& Wood, D. (1999a). Registration and management of smoking behaviour in patients with coronary heart disease. The EUROASPIRE survey [see comments]. European Heart Journal, 20(22)، 1630-7.

Van Berkel, T. F. M. Boersma, H., Roos-Hesselink, J. W. Erdman, R. A., \& Simoons, M. L. (1999b). Impact of smoking cessation and smoking interventions in patients with coronary heart disease. A systematic review. European Heart Joumal, 20(24), 1773-82.

Van Berkel, T. F. M., Boersma, E., Erdman, R. M., Bolman, C., Deckers, J. W., \& Roos, J. W. (submitted-a). A new smoking cessation programme for coronary patients in the outpatient clinic: $A$ baseline description and problems with inclusion. Manuscript submitted for publication.

Van Berkel, T. F. M.. Erdman, R. A. M., Deckers, J.W., Roos Hesseling, J. W., De Vries, H., \& Boersma, E. (submitted-b). A smoking cessation intervention for patients with a coronary artery disease in an outpatient setting. Efficacy and predictors of success. Manuscript submitted for publication.

Velicer, W. F., DiClemente, C. C., Prochaska, J. O., \& Brandenburg, N. (1985). Decisional balance measure for assessing and predicting smoking status, Journal of Personality and Social Psychology, 48(5), 1279-89.

Velicer, W. F. Prochaska, J. O., Bellis, J. M., Diclemente, C. C., Rossi, J. S., Fava, J. L., Steiger, J. H.. (1993). An expert system intervention for smoking cessation. Addictive Behaviors, 18(3), 269-290.

Velicer, W. F., Prochaska, J. O., Rossi, 1. 5., \& Snow, M. G. (1992). Assessing outcome in smoking cessation studies. Psychological Bulletin, 111(1), 23-41.

Velicer, W. F., Rossi, J. S., Diclemente, C. C., \& Prochaska, J. O. (1996). A criterion measurement model for health behavior change. Addictive Behaviors, 21(5), 555-84.

Vermette, L., \& Godin, G. (1996). Nurses" intentions to provide home care: The impact of AIDS and homosexuality. AlDS Care, 8(4), 479-488.

Vietstra, R. E., Kronmal, R. A., Oberman, A., Frye, R. L., \& Killip, T. (1986). Effect of cigarette smoking on survival of patients with angiographically documented coronary artery disease. Report from the CASS registry, Journal of the American Medical Association, 255(8), 1023-7.

Voors, A. A., Van Brussel, B. L., Plokker, H. W., Ernst, S. M., Ernst, N. M., Koomen, E. M. Tijssen, J. G. \& Vermeulen, F. E. (1996). Smoking and cardiac events after venous coronary bypass surgery. A 15-year follow-up study. Circulation, 93(1), 42-7. 


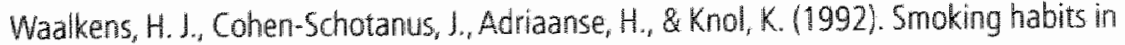
medical students and physiciams in Groningen, The Netherlands. European Respiratory loumal, 51), $49-52$.

Wells, K. B. Lewis, C. E., Leake, B., schleiter, M. K. \& Brook, R. H. (1986). The practices of general and subspecialty internists in counseling about smoking and exercise. Anerican loumal of Public Health, 76(8), 1009-13.

Wetter, D. W. Fiore, M. C., Gritz, E. R. Lando, H. A. Stitzer, M. L., Hasselblad, V., \& Baker, T. B. (1998). The Agency for Heath Care Policy and Research Smoking Cessation Clinical Practice Guideline: Findings and implications for psychologists. American Psychologist, 53(6), 657-669.

Whitelaw. 5., Baldwin, 5. Bunton, R., \& Flynn, D. (2000). The status of evidence and outcomes in Stages of Change research. Health Education Research, 15(6), 707-18.

Wiebing, M. A. \& Baan, B. (1997). De rol van de medische professie bij het terugdringen wan het roken [The role of the medical profession in reducing smoking]. Medisch contact, 52(25), 801-802.

Willemsen, M. C. \& De Vries, H. (1995). Evaluation of a smoking cessation intervention for Dutch employees consisting of self-help methods and a group program. Tobacco Control(4), 351-354.

Willemsen, M. C., De Vries, H., Van Breukelen, G. \& Genders, R. (1998). Long-term effectiveness of two Dutch work site smoking cessation programs. Health Education and Behavior, 254,418-35.

Willemsen, M. C., De Vries, H., Van Breukelen, G., \& Oldenburg, B. (1996). Determinants of intention to quit smoking among Dutch employees: the influence of the sacial emvironment. Preventive Medicine, 25(2), 195-202.

Windsor, R., Baranowski, T., Clark, N., \& Cutter, G. (1994). Evaluation of Health Promotion, Health Education, and Disease Prevention Programs (2th ed.). California: Mayfield Publishing Company.

Windsor, R. A., Boyd, N. R. \& Orleans, C. T. (1998). A meta evaluation of smoking cessation intervention research among pregnant women: improving the science and art. Health Education Research, 13(3), 419-38.

World Health Organization. (1992). International statistical dassification of diseases and related health problems. 10 th revision. Geneva: WHO.

World Health Organization. (1994). Cardiovascular disease. Risk factors: new areas for research (technical report series no. 841). Geneva: WHO.

World Health Organization. (1999). World Health Report: Making a Difference. Geneva: WHO.

Yin, R. K. (1979). Changing Urban Bureaucracies: How New Practices Becone Routinzed. Lexington: Mass, Lexington Books.

Zhu, S. H., Stretch, W. Balabanis, M., Rosbrook, B., Sadler, G., \& Pierce, J. P. (1996). Telephone counseling for smoking cessation: Effects of single-session and multiple-session interventions. Joumal of Consulting and Cinical Psychology, 64(1), 202-211. 


\section{SUMMARY}

In the Netherlands, cardiovascular disease is the leading cause of death, and a great majority of deaths from this type of disease are caused by coronary heart diseases. Research shows that lifestyle factors, such as smoking, are partly responsible for the development and progression of these diseases. For patients. who are already suffering from coronary heart disease, smoking cessation improves the prognosis more than any other treatment. In the Netherlands, no consistent attention had been paid to the smoking behavior of cardiac inpatients at the time this PhO project started. Therefore, a smoking cessation protocol was developed and tested at the cardiology wards of eleven Dutch hospitals. The present thesis describes the studies, that were carried out within the scope of this project.

Chapter one starts with a description of the so-called $A B C$ planning model for health education, since this model functioned as the framework for the project described in this thesis. Subsequently, the health problem of cardiovascular disease is analyzed and the development of the educational protocol for smoking cessation is described. The initiatives employed to anticipate on a future embedding of the protocol into daily hospital practice are outlined. As such, the chapter illustrates the work on the project from the start.

The probiem analysis shows that a substantial proportion of the patients who suffer from coronary heart disease persist in smoking, even though most patients admitted with coronary heart disease are willing to quit smoking. The chapter also shows that the smoking behavior of cardiac inpatients has so far been insufficiently addressed during hospital admission. Earlier research shows that nurses and cardiologists consider it their task to address patients' smoking behavior and to support them in smoking cessation. They are, however, pessimistic about the effects of their efforts. In addition, they report that they have insufficient knowledge and skills to provide patients with adequate guidance.

The key intervention described in the present thesis consists of an educational protocol allowing nurses and cardiologists working at cardiology wards to guide patients systematically in their attempts to stop smoking. An educational protocol entifled Minimal Intervention Strategy (MIS), which had been found to be effective in earlier research in the General Practice, served as the blueprint for the intervention. The essential elements of the protocol as adapted for cardiac impatients (called C-MIS) are: (1) a recommendation to quit smoking, provided by the cardialogist; (2) a counseling session with a ward nurse; (3) the provision of self-help materials; (4) aftercare after discharge from the hospital. A basic assumption of the protocol is that smoking cessation can be regarded as a process in which smokers progress through several phases, ranging from no motivation to change to the internalization of the new (non-smoking) behavior. 
Chapter wo describes the results of a cross-sectional study comparing patients in the various phases of change with respect to their psycho-social determinants. The study inwolved patients admitied to cardiology wards.

The results show that patients who do not smoke during hospital admission need to be divided into those who are willing to refrain from smoking after hospital dischar ge (intermally motivated actors) and those who are planning to resume smoking (externally motivated actors). The latter group refrain from smoking during hospital admission because the situation does not allows them to smoke. Internally motivated actors were found to perceive more pros of quitting, experience stronger social support to stop smoking and to be more convinced of their ability to refrain from smoking than externally motivated actors.

On the whole, the so-called $\theta$ pattern, which stresses the relative importance of three majo psycho-social determimants (attitude, social influence and self-efficacy expectations) for the various motivational phases, was confirmed for cardiac inpatients. In line with this $\varnothing$ pattern, internally motivated actors had stronger self-efficacy expectations than precontemplators and contemplators, while the latter two groups did not differ in their self-efficacy expectations. The perception of social support increased steadily from the precontemplation phase to the action phase. Contemplators and internally motivated actors had a comparable positive attitude toward quitting. In contradiction to the pattern, however, precontemplators were not significantly less convinced of the positive consequences of quitting than contemplators, while the outcomes provided strong indications into this direction. With respect to the psychosocial determinants, externally motivated actors were comparable to contemplators, and therefore fitted into the $\oslash$ pattern as contemplators. Finally, the findings showed that internally motivated actors were more convinced of the relationship between their disease and their own (smoking) behawior than patients in the other motivational phases of change.

The chapter ends with suggestions for the development or improvement of smoking cessation interventions for cardiac inpatients. Based on the $\varnothing$ pattern, it is advisable to tallor any health education efforts to the phase in which a patient is situated. In addition to the customary phases of change, an extra distinction needs to be made, namely between externally and internally motivated action. Precontemplators will benefit from motivational information emphasizing the positive consequences of quitting. Contemplators and externally motivated actors will benefit from information on how to cope with high risk situations, while for internally motivated actors, specific relapse prevention techniques need to be emphasized. Furthermore, precontemplators, contemplators and externally motivated actors in particular need to gain insight into the relationship between their smoking behavior and the development, progression and curre of the heart disease. 
Chapter three describes the effects of the protocol on smoking cessation. It concerns the effects that were measured thee months after the patients were included in the study. The study was carried out among 789 patients who were hospitalized with coronary heart disease and had smoked prior to admission. In five hospitals, patients were assisted by means of the C.MIS protocol (experimental group), while patients admitted to the cardiology wards of six other hospitals received the usual care (control groupl. In practice, the latter meant that hardly any attention was paid to patients' smoking behavior.

Outcome measures in terms of smoking behavior were assessed by means of questionnaires that were mailed to patients' home addresses three months after inclusion in the study. The questionnaire also included questions about the degree to which the smoking behavior had been addressed during hospital admission (process evaluation. A baseline measurement was assessed at the beginning of the hospital admission.

The results, corrected for covariates induding differences in baseline characteris. tics between the experimental and control groups, showed a significant intervention effect. Patients in the experimental group were more often abstinent from smoking than patients in the control group. This was found for point prevalence abstinence (not smoked during the last seven days) as well as for continuous abstinence (not smoked during the last three months). If the $22 \%$ dropouts were categorized as smokers (intention-to-treat procedure), only the intervention effect on point prevalence abstinence remained significant.

The process evaluation shows that mast expermental patients had received the intervention. The in haspital guidance had, however, been more frequently implemented in accordance with the protocol than the aftercare after discharge from hospital. Therefore, it was ascertained that the implementation of the protocol needs to be improved. Patients indicated that they appreciated the way their smoking habits were addressed.

Considering the results of the intention-to-treat analysis as the appropriate standard, mainly because experimental patients were less likely to return the cuestionnaire, it is concluded that the intervention is only effective on point prevalence abstinence after three months.

Chapter four examines the effects of the intervention after twelve months. The study involved the same sample of patients described in chapter three. In comparison with the usual care and after correction for covariates including baseline differences, the intervention resulted in higher point prevalence abstinence and continuous abstinence. These effects disappeared, however, if the $27 \%$ dropouts were categorized as smokers. 
Based on the assumption that a correct estimation of the effect requires an intention-to-treat analysis, it is concluded that there were no detectable intervention effects after twelve months.

Chapter five describes an empirical study among the nurses of the wards that served as the experimental group in the effectiveness study (described in chapters three and four). This survey studied the nurses' intentions to continue to work with the proto$\mathrm{col}$, and their underlying motives. The study aimed to find indications for methods to stimulate the diffusion of the intervention, if proven effective, in a non-research setting.

Most nurses were positive about the intervention and intended to continue using the protocol in the future. They were, however, not convinced that more patients would quit as a result of the new approach. Several parts of the protocol, like assessing a patient's motivation to quit and discussing the barriers perceived by a patient, were found to be difficult to implement. Nurses with strong intentions to continue to use the protocol perceived more positive consequences of the protocol use and found it less complex to carry out. The chapter ends with recommendations for the dissemination of the protocol and for related subjects, such as nurses ${ }^{t}$ training.

Chapter six outlines the most important findings of the individual studies. Further" more, methodological limitations of the studies are described, followed by recommendations for further research. On the basis of the studies described in this thesis, several conclusions are formulated. First, the intervention is effective in the short term (after three months\%, but the effect has disappeared after twelve months. Second, the implementation of the protocol needs to be improved. In this respect, it is also concluded that the content of the aftercare needs substantial improvement. Finally, it is concluded that it is desirable to study whether an improved protocol and improved implementation would lead to more quitting and better maintenance of non-smoking behavior among patients. 


\section{SAMENVATTING}

Hart- en vaatziekten wormen de belangrijkste doodsooraak in Nederland en veruit het grootste deel wan de totale sterfte aan deze zlekten wordt veroonaakt door coronaire harziekten. Onderzoek laat zien dat leefstijlfactoren, waaronder roken, mede verantwoordelijk zijn voor het antstaan van deze ziekten. Voor rokers die lijden aan een coronaire hartziekte, is stoppen de meest effectieve manier om de prognose te verbeteren.

in Nederland ontbrak het tot op heden aan structurele aandacht voor het rookgedrag van in het ziekenhuis opgenomen hartpatiënten. Daarom is een woorlichtingsprotocol ontwikkeld en uitgetest op de cardiologieafdelingen van elf Nederlandse ziekenhuizen. Dit proefschrift beschriftt de onderzoeken die in dit kader zijn uitgevoerd.

In hoofdstuk één wordt earst het ABC-planningsmodel voor planmatige gezondheidsvoorlichting beschreven. Dit model heeft als raamwerk gefungeerd bij de opzet en planning van het project dat onderwerp is wan dit proefschrift. Daarop volgt een analyse van het gezondheidsprobleem hart-en vaatziekten en een beschrijving van de totstandkoming van het voorlichtingsprotocol C-MIS. Er wordt tevens melding gemaakt van de initiatieven die zijn genomen met het oog op de toekomstige inbedding van de interventie in de dagelijkse ziekenhuispraktijk. Het hoofdstuk illustreert hoe en wanaf de start wan het project volgens het ABC-planningsmodel is gewerkt. De probleemanalyse laat zien dat een groot deel van de mensen die lijden aan een hartziekte blift roken. Toch blijkt het merendeel van de patiënten die met een hartziekte in het ziekenhuis zijn opgenomen, wel gemotiveerd te zijn om te stoppen met roken. Tevens is beschreven dat werpleegkundigen en cardiologen tot op heden onvoidoende aandacht besteden aan het rookgedrag van deze patienten. Eerder onderzoek laat zien dat verpleegkundigen en cardiologen het well als hun taak beschowwen om het rookgedrag met patiënten te bespreken en hen te ondersteunen bij het stoppen. Het effect van de inspanningen om het stopgedrag te beinvloeden schat men echter pessimistisch in. Bovendien denkt men over onvoldoende kennis en vardigheden te beschikken om patienten adequate ondersteuning te bieden.

De interventie die in dit proefschift centraal staat, is een voorlichtingsprotocol waarmee verpleegkundigen en cardiologen van de afdeing cardichogie patiënten kunnen ondersteunen bij het stoppen met roken. Het in de huisartsenpraktijk effectief gebleken voorlichtingsprotocol "Minimale Interventie Strategie" (de MS) fungeerde hierbij als ba5ils. In hoofdlijn bestaat de interventie woor hartpatienten (C-MIS genaamo) uit: (1) een door de cardioloog verschaft advies om te stoppen, (2) een begeleidingsgesprek met een afdelingswerpleegkundige, (3) schriftelike zelfhulpmaterialen. (4) nazorg na het ontslag uit het ziekenhuis. De interventie gaat evvan uit dat stoppen met roken een proces is 
waarbij rokers verschillende fasen doorlopen, lopend van geen motivatie om te veranderen tot het zich eigen maken van het niewwe (niet-roken) gedrag.

In hoofdstuk twee worden de resultaten beschreven van een cross-sectioneel onderzoek waarbij hartpatienten in de verschillende fasen van gedragsverandering met elkaar worden vergeleken op hun onderiggende psycho-sociale determinanten. Het betreft patiënten opgenomen op de afdeling cardiologie.

De groep patiënten die niet meer rookt sinds de opname in het ziekenhuis (actoren) is in twee groepen in te delen. Enerzijds patiënten die gemotiveerd zijn om niet meer te roken en die dit ook na ontslag uit het ziekenhuis willen wolhouden (intrinsiek gemotiveerde actoren), en anderzijds patiënten die weer gaan roken zodra de situatie hen dit toestaat (extrinsiek gemotiveerde actoren). De reden waarom de laatste groep niet meer heeft gerookt sinds de opname, is slechts het feit dat de situatie het niet toeliet. Intrinsiek gemotiveerde actoren zien meer voordelen van stoppen, ervaren meer sociale steun om niet meer te roken en zijn er sterker van overtuigd dat het hen zal lukken om blijvend te stoppen dan extrinsiek gemotiveerde actoren. Het $\varnothing$ patroon, dat het relatieve belang van drie belangriike psycho-sociale determinanten (attitude, sociale invloed en eigen-effectiviteitsverwachtingen) per fase van gedragsverandering aangeeft, wordt in het onderzoek grotendeels bevestigd voor hartpatiënten. Overeenkomstig dat patroon hebben intrinsiek gemotiveerde actoren hogere eigen-effectiviteitsverwachtingen dan precontemplators en contemplators. De laatste twee groepen verschillen niet in hun eigen-effectiviteitsverwachtingen. De perceptie van sociale steun stijgt geleidelijk van de precontemplatiefase maar de actiefase. Contemplators en intrinsiek gernotiveerde actoren hebben een vergelijkbare positieve attitude tegenover stoppen. Precontemplators zijn in tegenstelling tot wat het $\varnothing$ patroon verondersteit, niet significant minder overtuigd van de voordelen van stoppen met roken dan de contemplators, hoewel de uitkomsten wel sterk in die richting wijzen. Extrinsiek gemotiveerde actoren zijn wat betreft de psycho-sociale determinanten vergelijkbaar met mensen in de contemplatiefase, en passen als zodanig in het $\varnothing$ patroon.

Het hoofdstuk laat tevens zien dat intrinsiek gemotiveerde actoren sterker overtuigd zijn van de relatie tussen hun klachten en het rookgedrag dan mensen in de overige fasen. Het hoofdstuk wordt afgesloten met suggesties voor de ontwikkeling van een begeleidingspratocol om hartpatiënten te andersteunen bij het stoppen met roken. Zo is het op basis van het gevonden patroon aanbevelenswaardig om de voorlichting af te stemmen op de fase van gedragsverandering warin de patiënt zich bevindt. Hierbij dienen niet alleen de gebruikelijke groepen te worden onderscheiden, maar dient tevens verschil te worden gemaakt tussen extrinsiek en intrinsiek gemotiveerde actoren. Precontemplators hebben met name baat bij motiverende informatie, waarbij het accent moet liggen op de voordelen van stoppen met roken. Contemplators en extrinsiek gemotiveerde actoren zijn met mame gebaat met informatie over hoe ze met moeilijke situaties om kunnen gaan. Voor intrinsiek gemotiveerde actoren 
moet het accent liggen op het aanleren van specifieke technieken om terugval in het oude rookgedrag te woorkomen. Verder moeten met name precontemplators, contemplators en extern gemotiveerde actoren inzicht krigen in de relatie tussen roken, het ontstaan, het voortduren en het herstel van de hartziekte.

In hoofdstuk drie worden de effecten van het protocol op het stopgedrag van patiènten beschreven. Het betreft effecten die zijn gemeten drie maanden nadat de patiënten in het onderzoek zijn opgenomen. Het effectonderzoek is uitgevoerd onder 789 patiënten, die met hartklachten waren opgenomen op de verpleegaldeling cardiologie en rookten voor opname. Voor het onderzok is een quasi-experimenteel design met voor-en nametingen gebrukt. op de cardiologieafdelingen van vijf ziekenhuizen zijn de patiënten begeleid met de CMIS (die experimentele groep), terwifi nen op de cardiologieafdelingen wan zes andere ziekenhuizen de gebruikelijke voorlichting kreeg (de controlegroep). Dit laatste betekent in de praktijk dat er nauwelijks aandacht wordt besteed aan het rookgedrag van patiënten.

Om het effect van de interventie vast te stellen, kregen alle patienten drie maanden na toezegging van hun deelname een vragenlijst thuisgestuurd (nameting), waarop men moest invullen of men nog rookte. In deze vragenlijst werden tevens vragen gesteld over de mate warin het rookgedrag tijdens de opname ter sprake kwam (de procesevaluatie). Aan het begin van de opname werd een schriftelije voormeting afgenomen.

De resultaten, waarbij er is gecorrigeerd voor kenmerken waarop de experimentele- en controlegroep bij aanwang van het onderzoek van elkaar werschilden en voor factoren die het rookgedrag mogelijk ook zouden beïmoeden (covariaten), laten een significant interventie-effect zien. Patiënten in de experimentele groep zijin vaker gestopt met roken dan patiënten in de controlegroep. Dit geldt zowel voor de puntpreva lentie abstinentie meting (de afgelopen zeven dagen niet gerookt) als woor de continue abstinentie (de afgelopen drie maanden niet gerookt). Wanneer de $22 \%$ dropouts (mensen die de vragenligst niet hebben teruggestuurd) als rokers gecategori seerd worden, wordt er alleen een interventie effect op puntprevalentie abstinentie waargenomen.

De procesevaluatie laat zien dat de meeste patienten in de experimentele groep ook daadwerkelijk met behulp van de C.MAS zijn begeleid. Het gedeelte van de begeleiding dat tijdens de opname plaatshad, was vaker uitgevoerd conform het protocol dan de nazorg na ontslag uit het ziekentuis. Daarom is de implementatie van het protocol voor verbetering vatbaar. Patienten geven aan de begeleidingswijze wolgens het protocol te waarderen.

Ervan uitgaande dat voor een juiste effectschatting de dropouts als rokers beschouwd dienen te worden, vooral omdat de patiënten uit de experimentele groep minder geneigd waren om de vragenlijst terug te sturen, wordt geconcludeerd dat de 
interveritie enkel positieve effecten heeft op de puntprevalentie abstinentie na drie maanden.

Hoofdstuk vier gaat in op de effecten van de interventie na twaalt maanden. Het betreft hier dezelide groep patienten als beschreven in hoofdstuk drie. In vergelijking met de gebruikelijke begeleiding blikt de interventie, na een correctie voor verschillen in uitgangssituatie van patienten, te resulteren in een hogere puntprevalentie abstinentie en continue abstinentie. Deze effecten worden echter niet meer aangetoond als de $27 \%$ dropouts worden gecategoriseerd als rokers.

Ervan uitgaande dat voor een juiste effectschatting de dropouts als rokers beschouwd dienen te wonden, word: geconciudeerd dat er na twalf maanden geen effec ten zijn aangetoond.

Hoofdstuk wiff beschrift een empirische studie onder verpleegkundigen, die gedurende de effectstudie (beschreven in de hoofdstukken drie en vier) op de experimentele afdelingen werkten. In deze studie wordt onderzocht in hoeverre deze groep van plan is am in de toekomst met het protocol te blijuen werken, en welke redenen hieraan ten grondslag liggen. Deze studie tracht indicaties te vinden voor de bevordering van het gebruik van de interventie, mits deze effectief is gebleken, buiten de onderzoekssetting.

Het merendeel van de verpleegkundigen is positief over de C-MIS en is van plan het ook in de toekomst te blijven gebruiken. Wen is echter niet overtuigd dat meer patiënten stoppen als gevolg van de nieuwe begeleidingswijze. Verschillende onderdelen van het protocol, zoals het vaststellen van de motivatie van de patiënt om te stoppen en het adequaat ingaan op de door de patiënt ervaren bairières, vindt men moeilijk om uit te voeren. Verpleegkundigen die het protocol willen blijven gebruiken, zien er meer woordelen van en vinden het minder complex om het te gebruiken dan de verpleegkundigen die nog twijfelen of dit niet van plan zijn. Op basis van de bevindingen worden vervolgens aanbevelingen gedaan voor de toekomstige verspreiding van het protocol en hieraan gerelateerde onderwerpen zoals bijvoorbeeld de training voor verpleegkundigen.

In hootostuk zes zijn de belangrijkste bevindingen uit de afzonderlike studies uiteengezet en bediscussieerd. Tevens worden de methodologische beperkingen wan de studies beschreven, gevolgd door aanbevelingen voor toekomstig onderzoek. Op basis van de in dit proefschrift beschreven studies zijn een aantal conclusies geformuleerd. Ten eerste dat de C-MS effectief is op de korte terminn (na drie maanden), maar niet op de lange termiin (na twaalf maanden). Ten tweede dat het protocol in de toekomst beter ingevoerd dient te worden. In dit kader is tevens geconcludeerd dat de nazorg inhoudelijk substantiële verbetering behoeft. Tot slot is het wenselijk na te gaan of een verbeterd protocol en een goede invoering ervan er daadwerkelijk toe leiden dat er meer patiènten stoppen met roken en dit ook volhouden. 


\section{Notes}

1 Revascularization procedures: invasive pracedures (e.g. PTCA and CABG) intended to improve the blood supply to the endangered part of the heart muscle.

2 Coronary angioplasty (PTCA: Precutaneous Transluminal Coronary Angioplasty, better known as balloon dilatation): a palliative revascularization procedure, which involves dilating the coronary artery stenosis using a balloon which is introduced into the coronary artery via a catheter and inflated with a contrast medium.

3 Coronary artery bypass surgery ( $C A B G$ ): a palliative revascularization procedure, in which an extra vessel is inserted to bridge a vascular constriction in the coronary arteries.

4 Coronary event: in this thesis defined as all manifestations of diseases of the heart blood circulation system.

5 Stenosis of the coronary arteries: constriction of the blood vessels supplying the heart muscle. It results in the heart receiving an inadequate supply of oxygen (ischemia).

6 Since a genuine stage theory presumes qualitatively different non-reversible sta ges, we prefer to use the term phases. Therefore, in the remainder of this thesis, the term phases is used.

7 Although smoking in wards is prohibited by law, it is permitted to designate smoking areas within wards, e.g. in recreation rooms or in day rooms. Hospitals may also impose a total smoking ban. Because Dutch hospitals and wards may differ in their nonsmoking policy, patients in some wards may have been allowed to smoke, whereas others were not.

8 At the request of reviewers in the paper submission procedure, the term intervention group is used instead of experimental group in Chapter three.

9 Patients lost to follow-up included those who did nat retum the follow-up questionnaire. No distinction was made between those who were not longer willing to co-operate with the follow-up and those who could not be reached. 
10 Unequal variance T-tests were applied independently of the results of Leveneis Test for Equality of Variances, due to the unequal number of respondents in each group.

11 The response rate was calculated by dividing the number of questionnaires that were returned by the number of questionnaires requested by the IPCs.

12 The terms dropouts, patients lost to follow-up, and loss to follow-up are used interchangeably in this chapter and express the same (for details see footnote nine). 


\section{Abbreviations}

AP

ASE

CABG

CCU

CHD

Cl

C-MIS

CVD

FTND

Gps

IPCs

LCU

LHV

MCU

$\mathrm{MI}$

MIS

NRT

P-MIS

PTCA

SOC

Stivoro

$T M$

TPB angina pectoris

attitude social-influence selfefficacy

coronary bypass surgery

coronary care unit

coronary heart disease

confidence interval

minimal intervention strategy for cardiac inpatients

cardiovascular disease

fagerström test for nicotine dependence

general practitioners

internal program co-ordinators

low care unit

national association of general practitioners

medium care unit

myocardial infarction

minimal intervention strategy for general practitioners

micotine replacement therapy

minimal intervention strategy for coronary patients in the outpatient clinic cardiology

precutaneous transluminal coronary angioplasty

stage of change

Dutch foundation on smoking and health

transtheoretical model

theory of planned behavior 


\section{Curriculum vitaE}

Catherine Balman was born in Hapert on May $22^{\text {ns }}$, 1968. After graduating from secondary school, she started a psychiatric nurse's training, from which she graduated after 3.5 years. She then started studying at the Academy for Health Care $(\mathrm{Hbo}-\mathrm{V})$ in Breda, where she received her bachelor's degree. After working as a nurse for a period of time, she started studying Health Sciences at Maastricht University. In 1995 she graduated in Health Education with first class honors. Subsequently, she worked as a junior researcher at the department of Health Education of the same university. For four years, she spent two days a week working on the research project which is described in this thesis, while participating in several other studies during the rest of the week. The first of this involved a lifestyle study, which was carried out among Dutch youngsters and was commissioned by the Netherlands Heart Foundation. The second study was a cooperative project with the Erasmus University Rotterdam, in which she developed a part of a multicomponent smoking cessation intervention for patients diagnosed with coronary heart disease. This intervention was tested for its effectiveness in an outpatient cardiology setting. Her contribution consisted of developing and generating personalized computer-tailored smoking cessation feedback, which she composed in the form of letters that were sent to patients' home addresses. A third project involved a study on the use and impact of a Frequently Asked Questions checklist as a tool to prepare coronary outpatients for regular visits to their cardiologist. In the final year of her PhD project, she combined the study described in this thesis with lecturing at the Maastricht Inniversity. Since the $15^{\text {th }}$ of April 2000, she has been working as a lecturer at the Nethellands Open University, in the department of Social Sciences. 


\section{ACKNOWLEDGMENTS}

I would like to thank the Netherlands Heart Foundation and the Dutch Foundation on Smoking and Health for their financial support. I would also like to thank my promotor Hein de Vries for his supervision and my friend and family for their support.

Furthermore, I would like to express my gratitude to the following Dutch hospitals for their collaboration: University Hospital Maastricht, University Hospital Rotterdam. University Hospital Leiden, Rijnland Hospital, Rijnstate Hospital, Hospital De Heel, OLVG Hospital, Bosch Medical Center, St. Franciscus Hospital, Westrines Hospital, Medical Center Alkmaar.

I would also like to express a word of thanks to the members of the C-MIS project group, the working groupwest of the Netherlands Foundation on Cardiovascular nursing and to Gerard van Breukelen.

Finaliy, I would like to thank all other people who advised, supported or helped me during the research project and afterwards at the moment of writing this thesis. 